SFRVIÇO DEE POS-GRADIJAÇÃO DO ICMC-USPP

Data de Deprisito: $\quad 28.02 .20(12$

Assinatura:

\title{
Enlaçamentos bordantes
}

\author{
IIildebrane Augusto dos Santos
}

Orientador: Prof. Dr. José Eduardo Prado Pires de Campos

Disscrtação apresentada ao Instituto de Ciências Matemáticas e de Computação - ICMC-USP. como parte dos requisitos para obtenção do título de Mestre em Matemática.

USP - São Carlos

Fevereiro/2002 


\section{A Comissão Julgadora:}

Prof. Dr. José Eduardo Prado Pires de Campos

Prof. Dr. Oziride Nanzoli Neto

Prof Dr. Pedro Laiz Queiroz Pergher 
À Cormarie, Alice e Maria Antônia 


\section{Agradecimentos}

A Deus, sem o qual, nada é possível.

$\grave{A}$ Cormarie, Jefferson e Haroldo pelo amor, compreensão e apoio em todas as horas.

Ao Prof. I)r. José Eduardo P. Pires de Campos pela oportunidade que me deu de "crescer".

Aos meus amigos do IGCE-UNESP-Rio Claro e do ICMC-USP pelo incentivo e solidariedades constantes. Em especial à Elíris. Obrigada pelos conselhos, pcla ajuda nas disciplinas em que tive dificuldades, pelas várias vezes que me emprestou seu "ombro amigo" e por fazer da sua casa o meu outro lar.

Ao meu molhor amigo Sérgio pela amizade iniciada e consolidada durante o mestrado e pelos bons e maus momentos compartilhados.

Ao Esdras pelas noites de estudo e pela ajuda quando o assunto era computador.

A todos os meus Professores, por tudo que aprendi, por todo conhecimento que me ensinaram, tanto em nivel acadêmico como em nível pessoal. Fm especial aos Professores Eduardo Alex Hernandez Morales (USP - São Carlos), Marcelo José Saia (LSP - São Carlos) e Sérgio Roberto Nobre (UNESP - Rio Claro) pela preocupação e por acreditarem em mim.

À minha ex-oricntadora Prof ${ }^{a}$ Alice (UNESP - Rio Claro) por tudo o que fez por mim, sua exemplar orientação, pelo incentivo e acima de tudo por sua amizade.

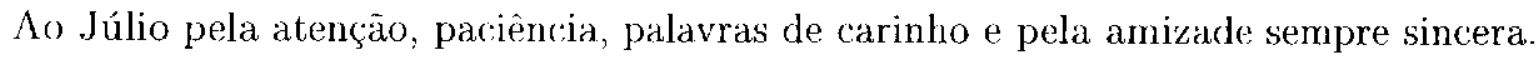

Á Maíra por ter agüentado o mou mal-humor nestes dois anos em que moramos juntas.

Ao Departamento de Matemática do ICMC-USP São Carlos.

$\grave{\Lambda}$ CAPES, pelo apoio financeiro.

A todas as pessoas que contuibuíram de maneira direta ou indireta para a realização deste trabalho. 


\section{Resumo}

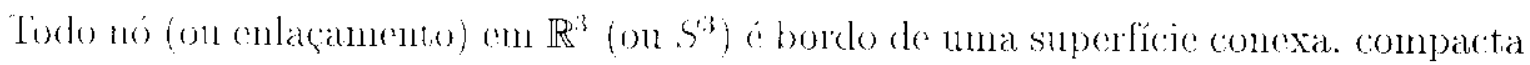

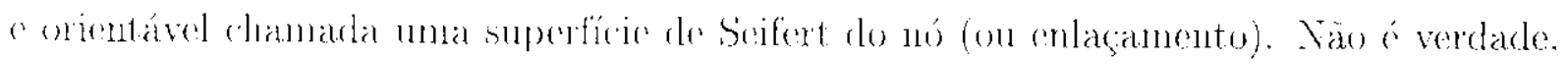

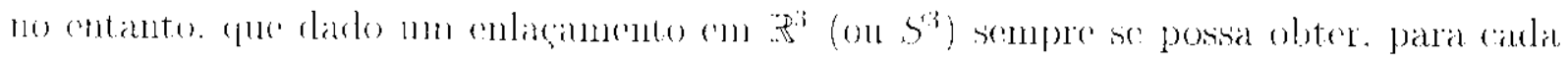

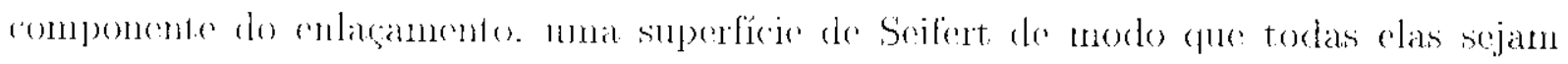

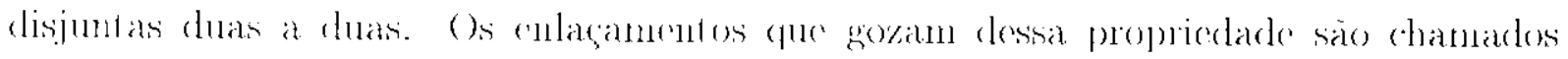

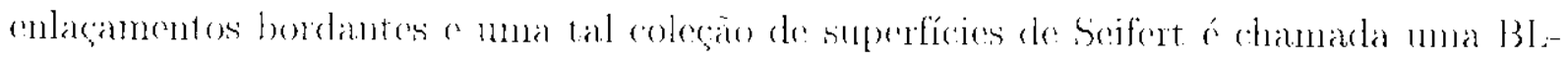
superfício do Soilint.

As diforemtes BL-superfícies de Soifert de um mesmo k-enlacamento bordante correspondent diferentes cisoes (splittings). (isto é. certos epimorfismos do grupo fundamental do k-enlacamento no grupo liver on $k$ geradores). a menos dos cobordismos apropriados.

() objetivo deste trabalho á apresentar un teorcuna que formece um método geomótrico

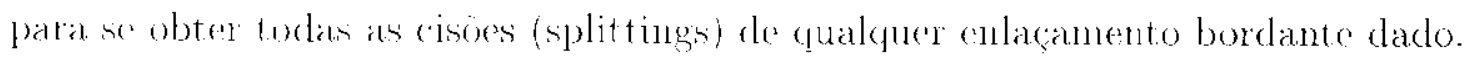




\section{Abstract}

Every knot (or link) in $\mathbb{R}^{3}$ or $S^{3}$ bounds a connected, oriented, compact surface called a beifert surface for the knot (or link). A boundary link is a link whose components bound dinjoint Scifert surfaces: we shall all the minon of such collection of surfaces; a BL-Seifert surface for the boundary link.

Different BL-Sedfert stnfiaces of a lac same: boundary link of k-components correspond to different splitting (that is certain epinomphism of the lundamental group of the k-link

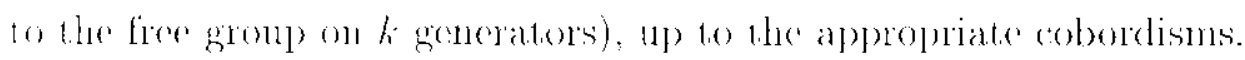

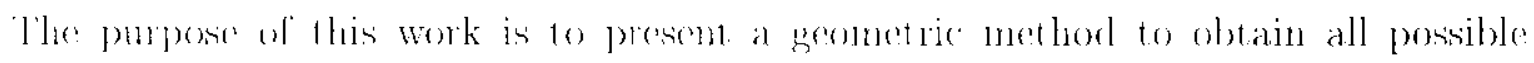
mplitting of any givon bommlary limk. 


\section{Sumário}

Introdução

1 Tranças 1

1.1 Tranças Geométricas. . . . . . . . . . . . . . . . . . . . . 1

1.2 Representação de Tranças como Automorfismos de Grupos Livres. . . . . . 7

2 Enlaçamentos $\quad 12$

2.1 A Apresentação de Wirtinger. . . . . . . . . . . . . . . . . . . . . 14

2.2 O grupo de cobordismo dos nós. . . . . . . . . . . . . . . . 18

2.3 Superfícies de Seifert. . . . . . . . . . . . . . . . . . . . . . 19

3 Enlaçamentos Bordantes $\quad 24$

3.1 Enlaçamentos Bordantes. . . . . . . . . . . . . . . . . . . 24

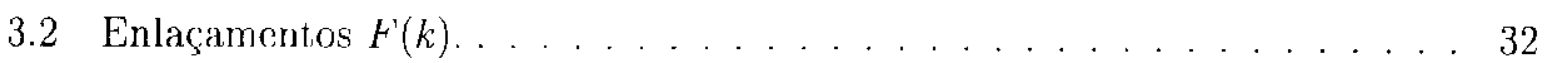

4 Enlaçamentos de Intervalos $\quad 36$

4.1 ○ Grupo $C_{1, k} \ldots \ldots \ldots \ldots \ldots$

4.2 A Ação de Habegger-Lin e o Cobordismo d-baseado. . . . . . . . . . . . 39

5 Enlaçamentos de Intervalos Bordantes $\quad 43$

5.1 Fnlaçamentos de Intervalos Bordantes. . . . . . . . . . . . . . . . . . 43

5.2 Enlaçamentos de Intervalos Bordantes e

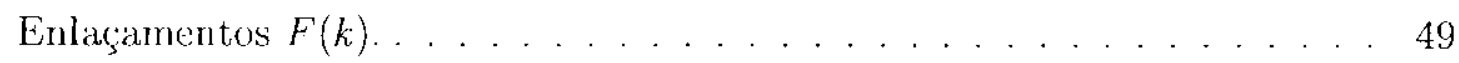

5.3 Enlaçamentos de Intervalos Bordantes e Tranças Puras. . . . . . . . . . 51

$\begin{array}{ll}\text { Referências Bibliográficas } & 65\end{array}$ 


\section{Introdução}

Um k-enlaçamento é um mergulho de $k$ cópias disjuntas de $S^{1}$ em $\mathbb{R}^{3}$. Existem várias relaçōes de equivalencia entre enlaçamentos tais como isotopia c cobordismo.

Geralmente é útil comparar, via nossas relações de equivalência, qualquer enlaçamento ao enlaçamento trivial padrão. Qualquer enlaçamento isotópico ao enlaçamento trivial padrão é chamado trivial; se o enlaçamento é cobordante ao trivial, dizemos que ele é slice.

Um conceito interessante, mais fraco do que trivialidade, ó o do enlaçamento bordante: um $k$-enlaçamento é trivial se ele se estende a um mergulho de $k$ cópias disjuntas de um disco $D^{2}$ em $\mathbb{R}^{3}$; um $k$-enlaçamento é bordante se ele sc cstende a um mergulho de $k$ superfícies compactas, conexas, orientáveis e disjuntas duas a duas, cada uma delas com bordo $S^{1}$. A união destas superfícies é chamada uma BL-superfície de Seifert do enlaçamento.

A condição de um enlaçamento ser bordante tem sua versäo algébrica como segue: um k-enlaçamento é bordante se, e somente se, existe um epimorfismo de $\Pi_{1}(X)$, onde $\Pi_{1}(X)$ é o grupo (fundamental do complemento) do enlaçamento, sobre o grupo livre em $k$ geradores, que leva certos clementos distinguidos, chamados meridianos, nos geradores. Chamaremos um tal epimorfismo de uma cisão (ou splitting) para o enlaçamento dado.

O resultado final deste trabalho (Teorema 5.3.3) fornece um método geométrico para se obter todas as cisões de um enlaçamento bordante. Tal método utiliza-se de uma ação do grupo das tranças puras no conjunto dos enlaçamentos de intervalos bordantes.

Lste trabalho está organizado como segue:

No capítulo 1 são introduzidas noções de tranças, o teorema da Apresentação de grupo de tranças de Artin e o t,corcma da Representaçào deste grupo.

No capítulo 2, descrevemos um procedimento para escrevermos uma apresentação do grupo de um nó em $\mathbb{R}^{3}$ : a Apresentaçäo de Wirtinger. Definimos o grupo de cobordismo dos nós e superfícies de Scifert.

No capítulo 3 são dadas as definiçôes e alguns resultados sobre enlaçamentos bordantes 
c cnlaçamentos $F(k)$, (isto é, um enlaçamento bordante munido de uma de suas cisões (split,tings)).

O capítulo 4 é dedicado ao estudo da açāo de Habegger-Lin e do cobordismo d-baseado, os quais serão utilizados no resultado final.

No capítulo 5 introduz-se a noçăo de enlaçamentos de intervalos bordantes, relacionandose tais enlaçamentos com os enlaçamentos $F(k)$ e demonstra-se o Teorema 5.3.3. 


\section{Capítulo 1}

\section{Tranças}

As tranças, como objeto matemático, foram introduzidas pelo matemático alemão Emil Artin em 1925. Artin propôs usar as tranças no estudo de nós e enlaçamentos. Existem agora aplicaçoes de tranças na topologia, na geometria, na teoria de singularidades, em sistemas dinâmicos, etc:

Começaremos com a definição de uma trança geométrica com $n$ cordas entre dois planos paralelos em $\mathbb{R}^{3}$.

Mostraremos o Teorema da Apresentação do grupo de tranças de Artin em termos de geradores e relações e o Teorema da Representação deste grupo, como um subgrupo do grupo de automorfismos do grupo livre com $n$ geradores.

\subsection{Tranças Geométricas.}

Em $\mathbb{R}^{3}$ escolhemos um sistemas de coordenadas $(x, y, z)$ no qual o eixo $z$ aponta verticalmente para baixo. Consideremos dois planos horizontais paralelos com coordenadas $z$ constante igual a 0 e 1 respectivamente. Chamemos o plano $z=0$ de plano superior e o plano $z=1$ de plano inferior. Marquemos $n$ pontos diferentes $P_{1}, \ldots, P_{n}$ em uma reta do plano superior e os projetemos verticalmente sobre o plano inferior, obtendo assim os pontos $P_{1}^{\prime}, \ldots, P_{n}^{\prime}$.

Definição 1.1.1. Um arco $\mathrm{em} \mathbb{R}^{3}$ é a imagem de um mergulho $\mathcal{A}_{i}: I \rightarrow \mathbb{R}^{3}$ do intervalo unitário $I-[0,1] \mathrm{em} \mathbb{R}^{3}$. Usaremos a mesma notação para o arco e para o mergulho correspondente. 
Definição 1.1.2. Uma trança (geométrica) com $n$ cordas ou uma n-trança $\beta$ é um sistema de $n$ arcos $\mathcal{A}=\left\{\mathcal{A}_{1}, \ldots, \mathcal{A}_{n}\right\}$ mergulhados em $\mathbb{R}^{3}$, onde o i-ésimo arco $\mathcal{A}_{i}$ está contido em $\mathbb{R}^{2} \times[0,1]$ e liga o ponto $P_{i}$ do plano superior ao ponto $P_{\tau(i)}$ do plano inferior, para alguma permulaçăo $\tau$ de $\{1, \ldots, n\}$ de modo que.

(1) Cada arro $\mathcal{A}_{i}$ intercepta cado plano paralelo intermediário aos planos superior e inferior cratamente uma vez.

(2) Os arcos $\left\{\mathcal{A}_{1}, \ldots, \mathcal{A}_{n}\right\}$ interceptam cada plano intermediário ans planos superior $e$ inferior em exatamente $n$ pontos diferentes.

A permutação $\tau$ é chamada a permutação da trança. $O$ arco $\mathcal{A}_{i}$ é chamado a i-ésima corda da trança.

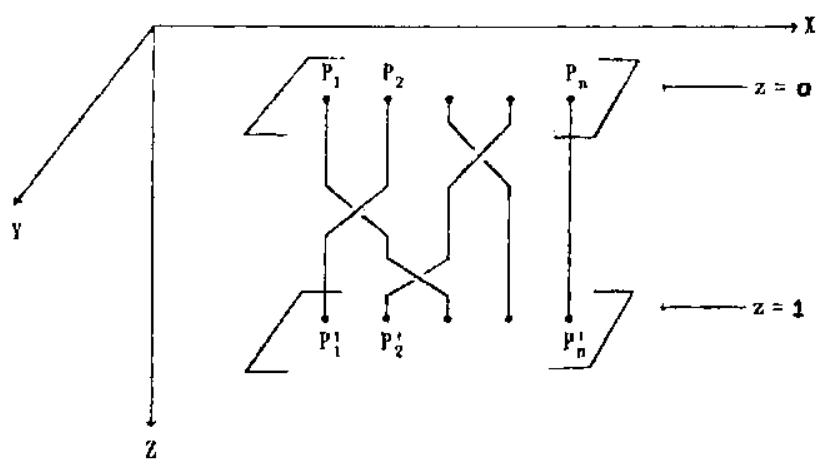

Figura 1

Vamos definir agora equivalência de tranças.

Definição 1.1.3. Duas n-tranças $\mathcal{A}^{0}=\left\{\mathcal{A}_{1}^{0}, \ldots, \mathcal{A}_{n}^{0}\right\}$ e $\mathcal{A}^{1}=\left\{\mathcal{A}_{1}^{1}, \ldots, \mathcal{A}_{n}^{1}\right\}$, com a mesma permutação $\tau$, são chamadas equivalentes (ou homotópicas) se existe uma homotopia, através de tranças, com permutação $\tau$, de $\mathcal{A}^{0}$ à $\mathcal{A}^{1}$. Em outras palavras, se existem $n$ aplicações contínuas $F_{i}: I \times I \rightarrow \mathbb{R}^{3}, 1 \leqq i \leqq n$ tal que

$$
\begin{array}{ll}
F_{i}(t, 0)=\mathcal{A}_{i}^{U}(t), & 0 \leq t \leq 1,1 \leq i \leq n \\
F_{i}(t, 1)=\mathcal{A}_{i}^{1}(t), & 0 \leq t \leq 1,1 \leq i \leq n \\
F_{i}(0, s)=P_{i}, & 0 \leq s \leq 1,1 \leq i \leq n
\end{array}
$$




$$
F_{i}(1, s)=P_{\tau(i)}^{\prime}, \quad 0 \leq s \leq 1,1 \leq i \leq n
$$

e tal que se definirmos $\mathcal{A}_{i}^{s}: I \rightarrow \mathbb{R}^{3}$ por $\mathcal{A}_{i}^{s}=F_{i}(t, s)$, então $\mathcal{A}^{s}=\left\{\mathcal{A}_{1}^{s}, \ldots, \mathcal{A}_{n}^{s}\right\}$ é uma $n$-trança com permutação $T$, para cada $0 \leqq s \leqq 1$.

A projeção (sombra) de uma trança $\beta \mathrm{em}$ um plano é dada como na figura 2 . Notemos que (através de equivalências) os cruzamentos das cordas ocorrem em níveis diferentes e que nos cruzamentos, as cordas que passam por cima e por baixo são indicadas.

$$
|\lambda|
$$

Figura 2

Para $1 \leq i \leq n-1$, denotemos por $\sigma_{i}$ a $n$-trança elementar, na qual a $i$-ésima corda passa por cima da corda $i+1$ una vez e todas as outras vão verticalmente em linha reta do ínicio ao fim.

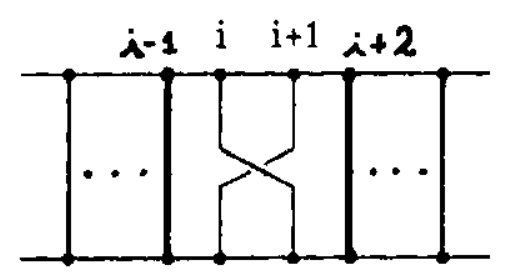

$\sigma_{\mathrm{i}}$

\section{Figura 3}

Seja $B(n)$ o conjunto de todas as classes de equivalência de $n$-tranças. Vamos definir uma estrutura de grupo neste conjunto.

Sejam $\beta_{1}$ e $\beta_{2}$ tranças com $n$ cordas. Definimos o produto (composição) de $\beta_{1}$ e $\beta_{2}$, denotado por $\beta_{1} \cdot \beta_{2}$, como segue: primeiro, coloquemos a trança $\beta_{2}$ em baixo da $\beta_{1}$ unindo o plano inferior de $\beta_{1}$ ao plano superior de $\beta_{2}$. Então removamos o plano que une estas 
duas tranças. Agora, vamos comprimí-las para que fiquem entre os planos $z=0 \mathrm{e} z=1$, c obteremos a trança $\beta_{1} \cdot \beta_{2}$.

Se $\beta_{1}$ e $\beta_{2}$ forem substituídas por tranças homotópicas $\beta_{1}^{\prime}$ e $\beta_{2}^{\prime}$ respectivamente, então podemos notar que o produto das tranças $\beta_{1} \cdot \beta_{2}$ e $\beta_{1}^{\prime} \cdot \beta_{2}^{\prime}$ são homotópicos. Logo, o produto de classes de equivalências de n-tranças está bem definido e determina um produto em $B(n)$.

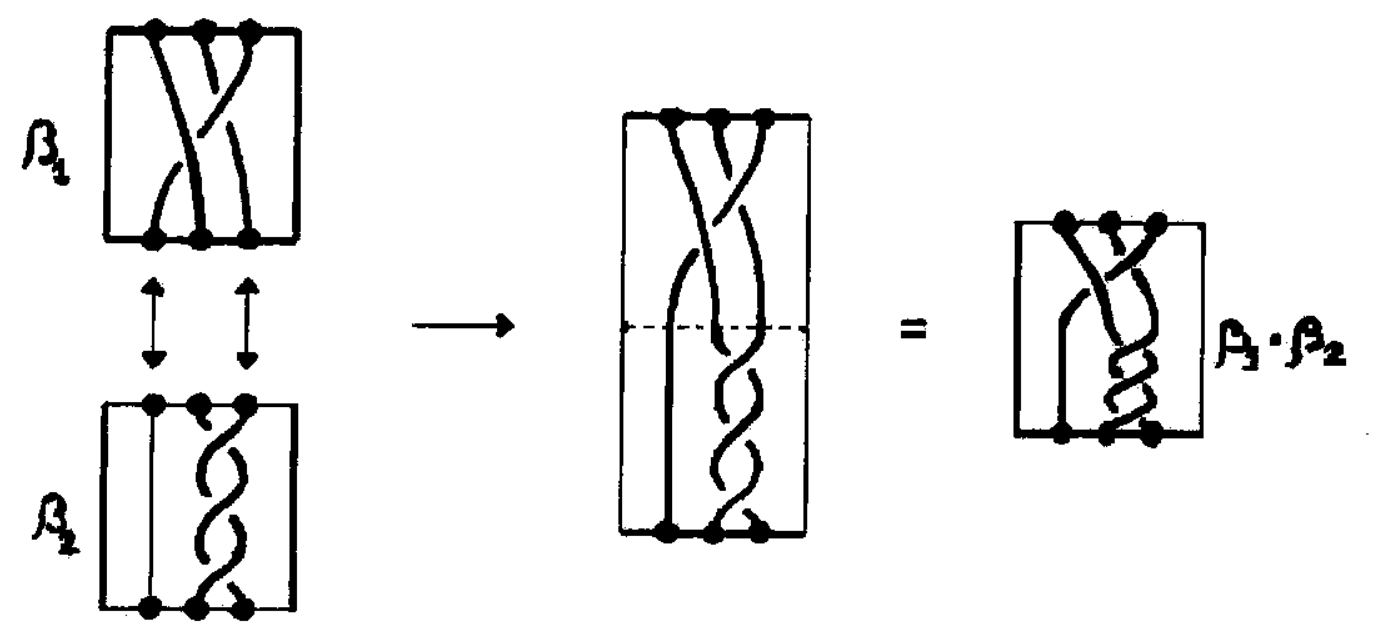

Figura 4

Facilmente pode-se observar a associatividade em B(n). Veja figura 5 .
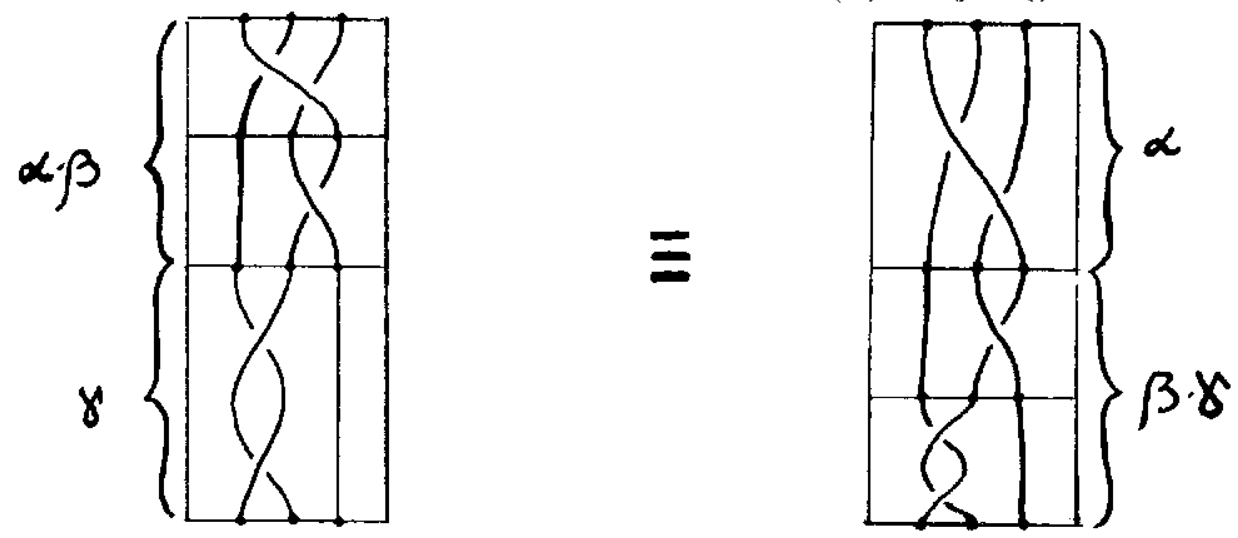

Figura 5

A trança trivial $\varepsilon$ é aquela em que todas as cordas vão verticalmente em linha reta do plano superior ao plano inferior. Notemos que $\varepsilon$ é o elemento neutro para o produto em $B(n)$. 


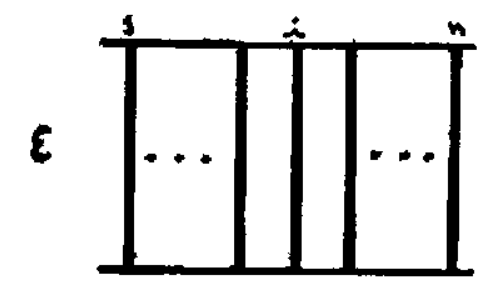

Figura 6
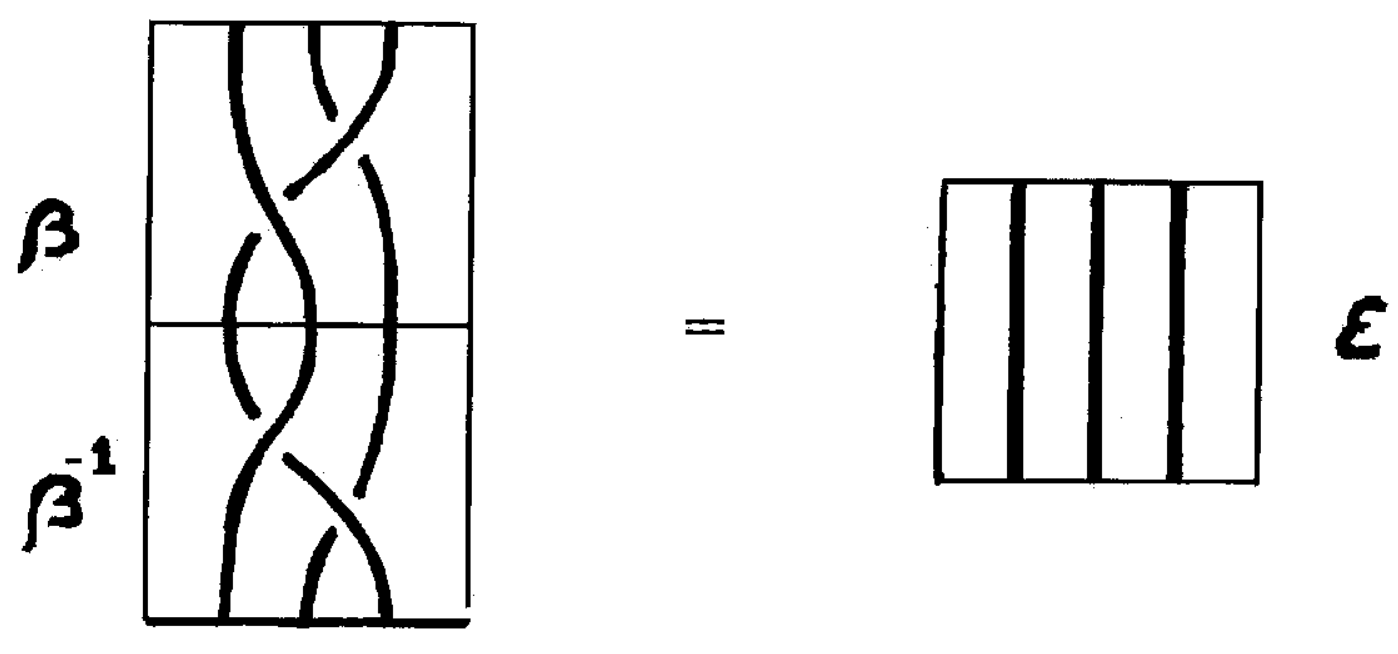

\section{Figura 7}

A trança inversa $\beta^{-1}$ da trança $\beta$ é a imagem refletida de $\beta$ com relação ao seu plano inferior.

Portanto, o conjunto $B(n)$ das classes de equivalência de $n$-tranças é um grupo. Este grupo é chamado o grupo de tranças de Artin com $n$ cordas.

Para a $n$-trança elementar $\sigma_{i}, 1 \leq i \leq n-1$, o elemento inverso $\sigma_{i}^{-1}$ é obtido invertendo o cruzamento da corda $i$ por cima da corda $i+1$ para o cruzamento da corda $i+1$ por cima da corda $i$.

Observação 1.1.4. Quando for claro no contexto, confundiremos uma trança com sua classe de equivalência, utilizando a mesma notação para ambas. Qualquer n-trança pode: ser escrita como um produto de n-tranças clcmentares $\sigma_{i}, 1 \leq i \leq n-1$, e de seus inversos. Logo, as $n$-tranças clementares $\sigma_{1}, \ldots, \sigma_{n-1}$ e seus inversos geram o grupo $B(n)$. Por exemplo, a trança $\beta$ com projeção na figura 2 pode ser escrita como o produto $\sigma_{2}^{-1} \sigma_{1}$.

Devemos olhar agora para as relações entre os elementos de $B(n)$. Primeiro notemos que 

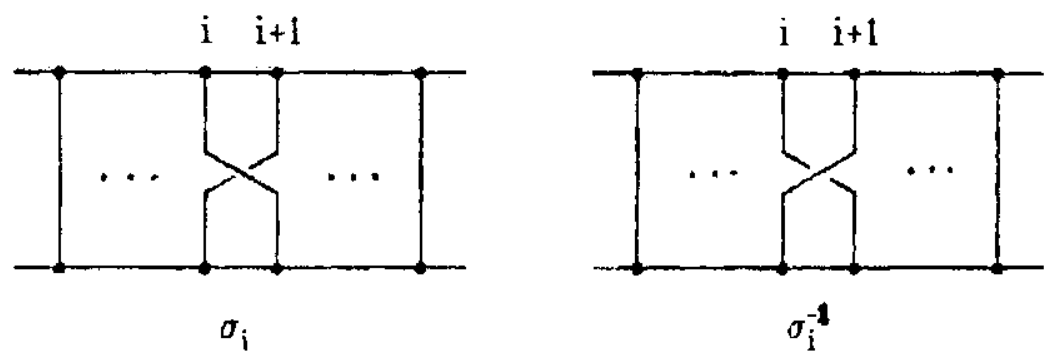

Figura 8

$$
\begin{aligned}
& \text { (1) } \sigma_{i} \cdot \sigma_{j}=\sigma_{\jmath} \cdot \sigma_{i} \text { para }|i-j| \geq 2,1 \leq i, j \leq n-1 \\
& \text { (2) } \sigma_{i} \cdot \sigma_{i+1} \cdot \sigma_{i}=\sigma_{i+1} \cdot \sigma_{i} \cdot \sigma_{i+1} \text { para } 1 \leq i \leq n-2 .
\end{aligned}
$$

As figuras abaixo ilustram as relações (1) e (2).
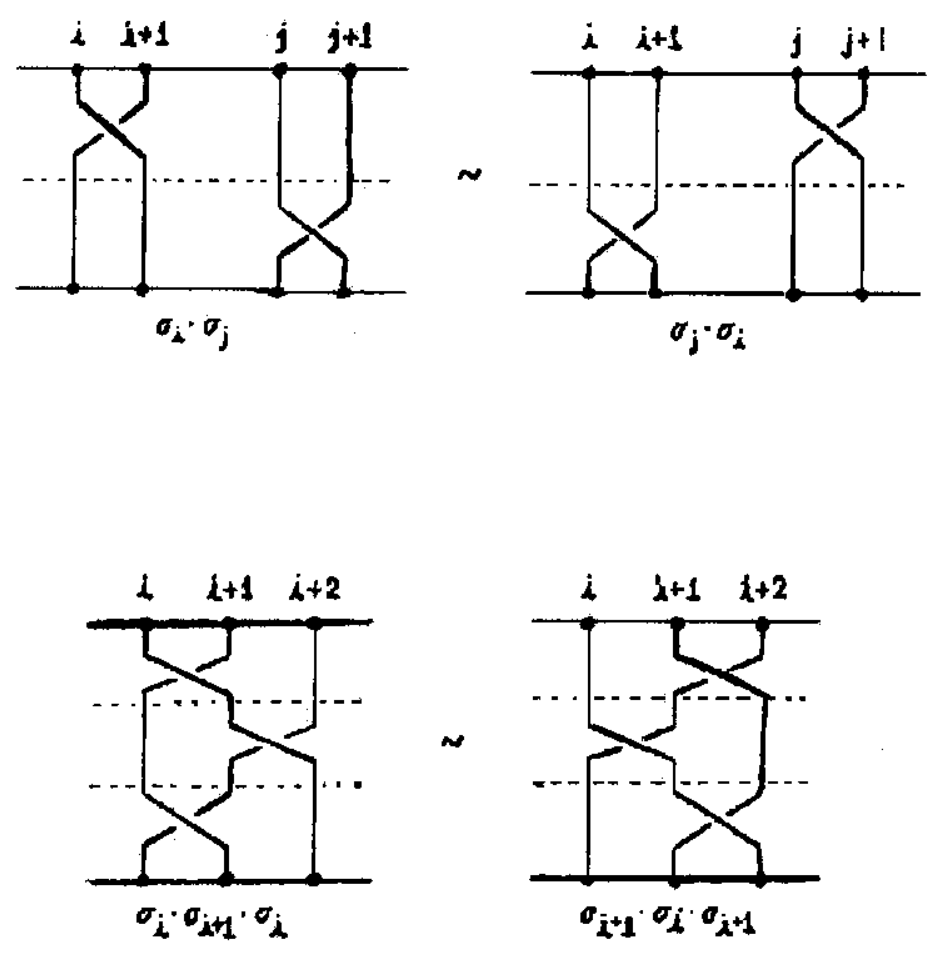

Figura 9

No primeiro artigo de Artin sobre grupos de tranças (1925), foi dada uma demonstração de que as relações (1) e (2) geravam todas as relaçōes entre os elementos de $B(n)$. Fnunciaremos este resultado como o Teorema 1.1.5 a seguir. 


\section{Teorema 1.1.5. Teorema da Apresentaçāo de Artin.}

O grupo $B(n)$ de tranças geométricas com $n$ cordas admite uma apresentação com geradores: $\sigma_{1}, \sigma_{2}, \ldots, \sigma_{n-1} \quad e$

relaçōes: $\quad(1) \sigma_{i} \cdot \sigma_{\jmath}=\sigma_{j} \cdot \sigma_{i}$ para $|i-j| \geq 2,1 \leq i, j \leq n-1$

(2) $\sigma_{i} \cdot \sigma_{i+1} \cdot \sigma_{i}=\sigma_{i+1} \cdot \sigma_{i} \cdot \sigma_{i+1}$ para $1 \leq i \leq n-2$.

Uma demonstração deste teorema pode ser encontrada em [[8] pág 18].

\subsection{Representação de Tranças como Automorfismos de Grupos Livres.}

Nosso objetivo nesta seção é provar que o grupo de tranças $B(n)$ é isomorfo a um subgrupo do grupo de automorfismos $\operatorname{Aut} F(n)$ do grupo livre $F(n)$ com $n$ geradores.

Teorema 1.2.1. Teorema da Representação de Artin.

Seja $F^{\prime}(n)$ o grupo livre em un conjunto de $n$ geradores $x_{1}, \ldots, x_{n}$ para um inteiro fixo $n \geq 1$ e seja AutF(n) o grupo de automorfismos de $F(n)$. Entấo $B(n)$ é isomorfo ao subgrupo de $A u t F(n)$ consistindo dos automorfismos $\bar{\beta}$ de $F(n)$ satisfazendo as seguintes condiçòes

$$
\begin{gathered}
\beta\left(x_{i}\right)=A_{i} x_{\tau(i)} A_{i}{ }^{1}, 1 \leq i \leq n \\
\text { e } \bar{\beta}\left(x_{1} \ldots x_{n}\right)=x_{1} \ldots x_{n}
\end{gathered}
$$

onde $\tau$ é uma permulação de $\{1, \ldots, n\}$ e todo $A_{i}$ pertence a $F(n)$, isto é, cada $A_{i}$ é uma palavra nos geradores $x_{1}, \ldots, x_{n}$.

Sob este isomorfismo, a trança elementar $\sigma_{i}, 1 \leq i \leq n-1$, corresponde ao automorfismo $\tilde{\sigma}_{i} \in \operatorname{Aut} F(n)$ definido por

$$
\begin{gathered}
\bar{\sigma}_{i}\left(x_{i}\right)=x_{i} x_{i+1} x_{i}{ }^{1} \\
\sigma_{i}\left(x_{i+1}\right)=x_{i} \\
\bar{\sigma}_{i}\left(x_{j}\right)=x_{j}, \forall j \neq i, i+1,
\end{gathered}
$$


e a permutaçäo $\tau$ para o automorfismo $\bar{\beta} \in A$ ut $F(n)$ correspondendo à trança $\beta \in B(n)$ é exatamente a permutação da trança.

Daremos a seguir uma versão resumida da prova deste teorema. Una versão mais detalhada pode ser encontrada em [[2] pág 30].

Sejam $P_{1}=(1,0,0), P_{2}=(2,0,0), \ldots, P_{n}=(n, 0,0)$ pontos no plano $z=0 \mathrm{e}$ $P_{1}^{\prime}=(1,0,1), P_{2}^{\prime}=(2,0,1), \ldots, P_{n}^{\prime}-(n, 0,1)$ pontos no plano $z=1$ os conjunto de pontos inicias e finais, respectivamente, para as tranças.

Sejam $P_{0}=(0,0,0), P_{0}^{\prime}=(0,0,1), \mathbb{R}_{0}^{2}$ o plano $z=0$ e $\mathbb{R}_{1}^{2}$ o plano $z=1$.

O grupo livre $F(n)$ com geradores $x_{1}, \ldots, x_{n}$ pode ser identificado com os grupos fundamentais dos planos perfurados

$$
\mathrm{IJ}_{1}\left(\mathbb{R}_{0}^{2} \backslash\left\{P_{1}, \ldots, P_{n}\right\}, P_{0}\right) \cong \Pi_{1}\left(\mathbb{R}_{1}^{2} \backslash\left\{P_{1}^{\prime}, \ldots, P_{n}^{\prime}\right\}, P_{0}^{\prime}\right)
$$

O gerador $x_{i}$ é representado em $\Pi_{1}\left(\mathbb{R}_{0}^{2} \backslash\left\{P_{1}, \ldots, P_{n}\right\}, P_{0}\right)$ pelo laço em $\mathbb{R}_{0}^{2} \backslash\left\{P_{1}, \ldots, P_{n}\right\}$ com ponto base $P_{0}$ que circunda $P_{i}$ uma vez no sentido anti-horário, como pode ser visto na figura 10. Analogamente, $x_{i}$ tem $1 \mathrm{~m}$ representante em $\mathrm{I}_{1}\left(\mathbb{R}_{1}^{2} \backslash\left\{P_{1}^{\prime}, \ldots, P_{n}^{\prime}\right\}, P_{0}^{\prime}\right)$.

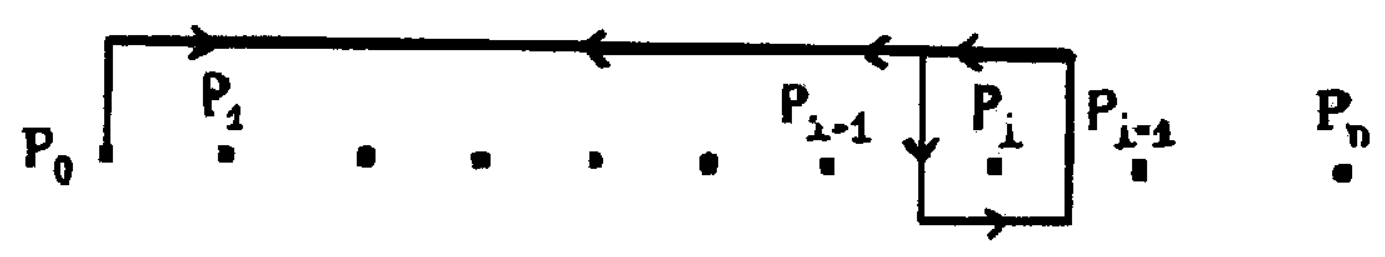

Figura 10

Seja $\beta$ uma trança em $\mathbb{R}^{3}$. Consideremos $\mathbb{R}^{2} \times I$ com as cordas da trança $\beta$ removidas. Correspondendo a $\beta$, podemos definir uma aplicação

$$
\bar{\beta}: F(n) \cong \Pi_{1}\left(\mathbb{R}_{0}^{2} \backslash\left\{P_{1}, \ldots, P_{n}\right\}, P_{0}\right) \rightarrow F(n) \simeq \Pi_{1}\left(\mathbb{R}_{1}^{2} \backslash\left\{P_{1}^{\prime}, \ldots, P_{n}^{\prime}\right\}, P_{0}^{\prime}\right),
$$

empurrando um laço l em $\mathbb{R}_{0}^{2} \backslash\left\{P_{1}, \ldots, P_{n}\right\}$, com ponto base $P_{0}$, para baixo ao longo dos espaços vazios deixados pelas $n$ cordas de $\beta$ em um laço $\bar{\beta}(l)$ ern $\mathbb{R}_{1}^{2} \backslash\left\{P_{1}^{\prime}, \ldots, P_{n}^{\prime}\right\}$ com 
ponto base $P_{0}^{\prime}$. A figura 11 ilustra o processo de obtenção de $\bar{\beta}$ a partir de $\beta$.
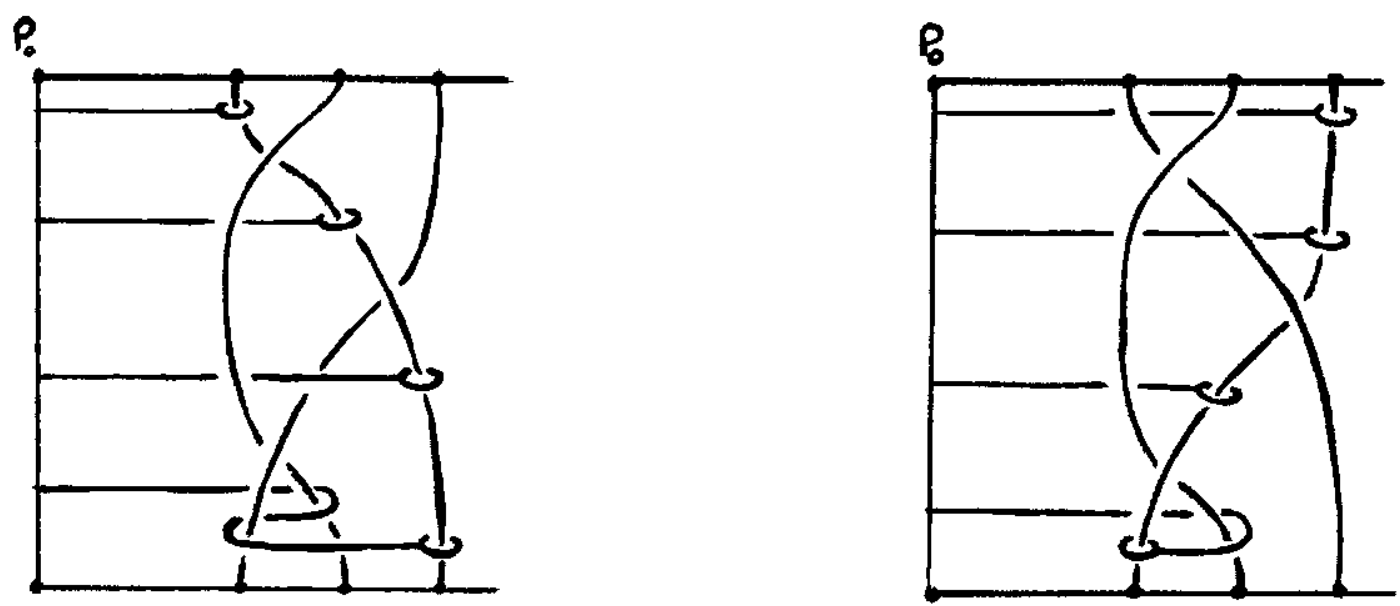

Figura 11

Este procedimento define uma aplicação $\bar{\beta}$ ern classes de homotopia conforme a

Afirmação 1: Se $l_{1}$ e $l_{2}$ são laços $\mathrm{em} \mathbb{R}_{0}^{2} \backslash\left\{P_{1}, \ldots, P_{n}\right\}$, com ponto base $P_{0}$, os quais são homotópicos relativamente a $P_{0}$, então $\bar{\beta}\left(l_{1}\right)$ e $\bar{\beta}\left(l_{2}\right)$ são homotópicos em $\mathbb{R}_{1}^{2} \backslash\left\{P_{1}^{\prime}, \ldots, P_{n}^{\prime}\right\}$ relativamente a $P_{0}^{3}$.

Afirmação 2: Se $l_{1}$ e $l_{2}$ são laços em $\mathbb{R}_{0}^{2} \backslash\left\{P_{1}, \ldots, P_{n}\right\}$, com ponto base $P_{0}$, então o produto dos laços $l_{1} \cdot l_{2}$ satisfaz $\bar{\beta}\left(l_{1} \cdot l_{2}\right)=\left(\bar{\beta}\left(l_{1}\right)\right) \cdot\left(\bar{\beta}\left(l_{2}\right)\right)$. Logo, $\beta$ é um homomorfismo.

Afirmação 3: $\bar{\beta}$ é um automorfismo.

Para provar esta afirmação precisamos encontrar um homomorfismo inverso à $\bar{\beta}$.

O homomorfismo inverso $\beta^{-1}$ é obtido empurrando um laço com ponto base $P_{0}^{\prime}$ em $\mathbb{R}_{l}^{2} \backslash\left\{P_{1}^{\prime}, \ldots, P_{n}^{\prime}\right\}$ para cirma, ao longo dos espaços vazios deixados pelas cordas de $\beta$, até obtermos um laço enn $\mathbb{R}_{0}^{2} \backslash\left\{P_{1}, \ldots, P_{n}\right\}$ com ponto base $P_{0}$, ou seja, $\bar{\beta}^{-1}=\overline{\beta^{-1}}$.

Da definição de homotopia de tranças segue que

Afirmação 4: Se $\beta^{\prime}$ é uma trança com $n$ cordas homotópica a $\beta$, então $\bar{\beta}^{\prime}=\bar{\beta}$.

A afimação 4 garante que se obtém uma aplicação bem definida de $B(n)$ em $A u t F(n)$.

Afirmação 5: A aplicação $B(n) \rightarrow A u t F(n)$, que associa uma trança $\beta \in B(n)$ ao automorfismo $\bar{\beta} \in \operatorname{Aut} F(n)$ é um homomorfismo. Em outras palavras, se $\beta_{1}$ e $\beta_{2}$ são $n$-tranças quaisquer, então $\overline{\beta_{1} \cdot \beta_{2}}=\bar{\beta}_{1} \bar{\beta}_{2}$. 
Afirmaçāo 6: $O$ automorfismo $\bar{\beta} \in A u t F(n)$, correspondendo a $n$-trança $\beta \in B(n)$ satisfaz $\bar{\beta}\left(x_{1} x_{2} \ldots x_{n}\right)=x_{1} x_{2} \ldots x_{n}$.

Como cada $x_{i}, 1<i \leq n$, é representado por um caminho fechado em $\mathbb{R}_{n}^{2} \backslash\left\{P_{1}, \ldots, P_{n}\right\}$ com ponto base $P_{0}$, que circunda $P_{i}$ uma vez no sentido anti-horário, o produto $x_{1} x_{2} \ldots x_{n}$ é dado pelo caminho

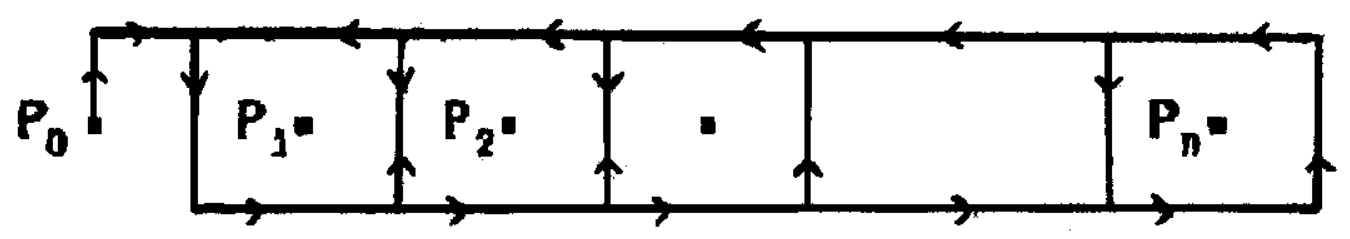

Figura 12

ou seja, os pontos $P_{1}, P_{2}, \ldots, P_{n}$ săo circundados uma vez. Mas este caminho é homotópico ao caminho

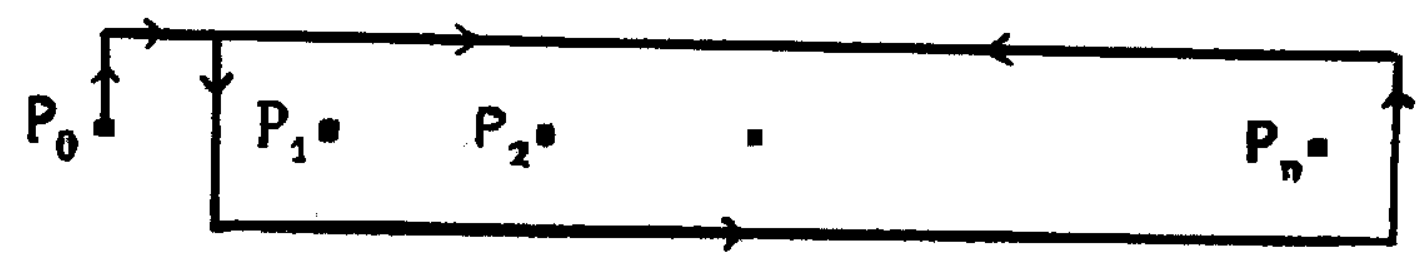

Figura 13

O mesmo procedimento é realizado para o caminho fechado $\bar{\beta}\left(x_{1}\right) \bar{\beta}\left(x_{2}\right) \ldots \bar{\beta}\left(x_{n}\right)=$ $\bar{\beta}\left(x_{1} x_{2} \ldots x_{n}\right)$ em $\mathbb{R}_{1}^{2} \backslash\left\{P_{1}^{\prime}, \ldots, P_{n}^{\prime}\right\}$, o qual circunda urra vez $P_{1}^{\prime}, P_{2}^{\prime}, \ldots, P_{n}^{\prime}$ no sentido antihorário. Portanto, $\bar{\beta}\left(x_{1} x_{2} \ldots x_{n}\right)=x_{1} x_{2} \ldots x_{n}$.

Afirmação 7: Consideremos $\sigma_{i}, 1 \leq i \leq n-1$ a trança elementar com $n$ cordas. Então

$$
\begin{gathered}
\bar{\sigma}_{j}\left(x_{j}\right)=x_{j}, j \neq i, i+1 \mathrm{e} \\
\bar{\sigma}_{i}\left(x_{i+1}\right)=x_{i} .
\end{gathered}
$$

Além disso, quando empurramos um caminho que representa $x_{i}$ para baixo do longo da trança $\sigma_{i}$, ele passa na frente de $P_{i}^{\prime}$, como na figura $14 a$, obtendo-se um caminho que é homotópico ao caminho ilustrado em 146 que representa $x_{i} x_{i+1} x_{i}^{-1}$. Logo, 


$$
\bar{\sigma}_{i}\left(x_{i}\right)=x_{i} x_{i+1} x_{i}
$$

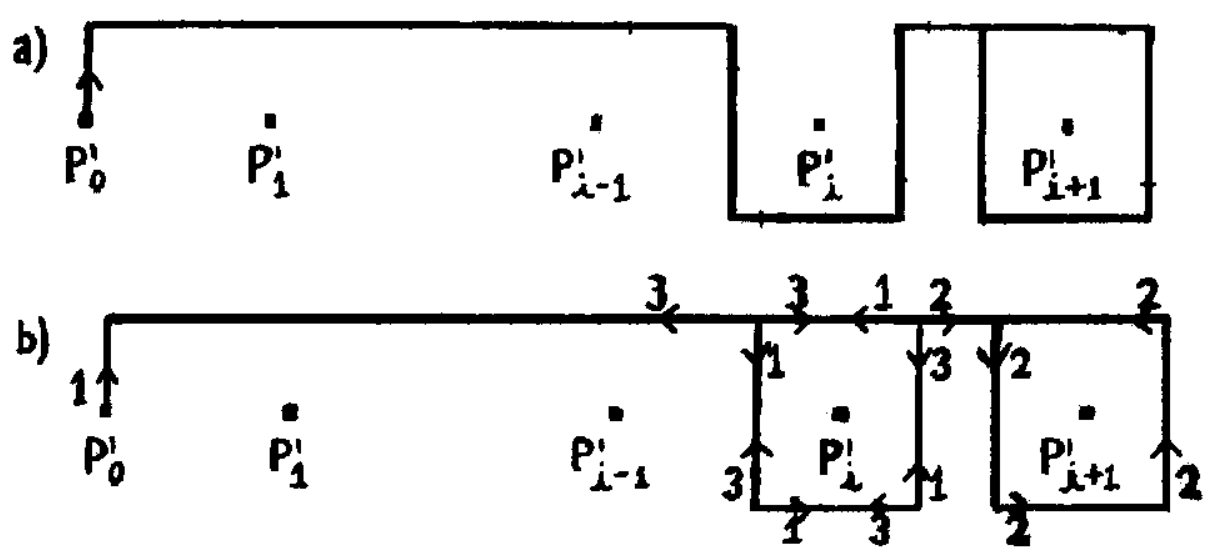

Figura 14

As flechas 1, 2 e 3 correspondem aos laços $x_{i}, x_{i+1}$ e $x_{i}{ }^{1}$, respectivamente, percorridas nesta ordem.

Afirmaçāo 8: $\bar{\beta}\left(x_{i}\right)=A_{\imath} x_{\tau(\eta)} A_{i}^{-1}, 1 \leq i \leq n$.

Desde que $\overline{\sigma_{i}^{-1}}=\left(\overline{\sigma_{i}}\right)^{-1}$, então

$$
\begin{gathered}
\overline{\sigma_{i}^{-1}}\left(x_{j}\right)=x_{j}, j \neq i, i+1 \\
\overline{\sigma_{i}^{-1}}\left(x_{i}\right)=x_{i \downarrow 1} \\
\overline{\sigma_{i}^{-1}}\left(x_{i+1}\right)=x_{i 11}^{-1} x_{i} x_{i+1} .
\end{gathered}
$$

Seja $\beta \in B(n)$ tal que $\beta=\sigma_{i_{1}}^{\epsilon_{1}} \ldots \sigma_{i_{k}}^{\epsilon_{k}} \operatorname{com} \epsilon_{j}- \pm 1,1 \leq j<k$. Então, pola afirmação $5, \quad \bar{\beta}=\overline{\sigma_{i_{1}}^{\epsilon_{1}}} \cdot \ldots \cdot \overline{\sigma_{i_{k}}^{\epsilon_{k}}}$. Segue que existem elementos $\Lambda_{i} \in F(n), 1 \leq i \leq n$ e uma permutação $\tau$ de $\{1, \ldots, n\}$ onde

$$
\bar{\beta}\left(x_{i}\right)=A_{i} x_{\tau(i)} A_{i}^{-1}, 1 \leq i \leq n
$$

Considerando-se a atuação de cada $\overline{\sigma_{i}^{+1}}$, vê-se que $\tau$ é a permutação da trança $\beta$.

Afirmação 9: Qualquer automorfismo em $\operatorname{AutF}(n)$, satisfazendo as condiçôes do teorema é realizado por uma única trança em $B(n)$.

Não daremos aqui a demonstração desta afirmação.

Assim, concluímos a prova deste trorema, obtendo o isomorfismo desejado. 


\section{Capítulo 2}

\section{Enlaçamentos}

Definição 2.0.2. Um enlaçamento é um mergulho (PL ou) diferenciável $K: S_{1}^{1} \cup \cdots \cup$ $S_{r}^{1} \rightarrow \mathbb{R}^{3}$ (ou $\left.S^{3}\right)$, onde cada $S_{i}^{1}, 1 \leq i \leq r$, é homeomorfa a $S^{1}$. Se $r=1$ o enlaçamento é chamado um nó.

Definição 2.0.3. Dois mergulhos $f_{0}, f_{1}: X \rightarrow Y$ são isotópicos se existe em mergulho

$$
F: X \times I \rightarrow Y \times I
$$

tal que $F(x, t)=(f(x, t), t), x \in X, l \in I$ com $f(x, 0)=f_{0}(x), f(x, 1)=f_{1}(x)$.

$F$ é chamada uma isotopia preservando nível conectando $f_{0}$ e $f_{1}$.

Definição 2.0.4. Dois mergulhıs $f_{0}, f_{1}: X \rightarrow Y$ săo ambiente isotópicos se existe uma isotopia preservando nível

$$
H: Y \times l \rightarrow Y \times I, \quad H(y, t)=(h(y, t), t),
$$

$\operatorname{com} f_{1}=h_{1} f_{0} \Leftrightarrow h_{0}=i d_{Y}$, onde $h_{t}(y)=h(y, t)$. Hé chamada uma isotopia ambiente.

Uma isotopia ambiente define uma isotopia $F$ conectando $f_{0}$ e $f_{1}$ por $F(x, t)=$ $\left(h_{i} f_{0}(x), t\right)$. A diferença entre as duas definições é a seguinte: uma isotopia move o conjunto $f_{0}(X)$ continuamente sobre $f_{1}(X) \mathrm{cm} Y$, sem dar atenção aos pontos de $Y$ que näo pertencem a $f_{t}(X), 0 \leq t \leq 1$. Lima isotopia ambiente requer que $Y$ mova-so continuamente com $f_{\ell}(X), 0 \leq t \leq 1$.

Definiçāo 2.0.5. Dois nós ou enlaçamentos $K$ e $K^{\prime}$ sâo equivalentes se eles săo ambiente isotópicos. 
Proposição 2.0.6. Sejam $K$ e $K^{\prime}$ nós em $S^{3}$. As seguintes afirmaçồes são equivalentes.

(1) Existe um homeomorfismo preservando orientação $f: S^{3} \rightarrow S^{3}$ que leva $K$ sobre $K^{\prime}, f(K)=K^{\prime}$.

(2) $K$ e $K^{\prime}$ sîo equivalentes (ambiente isotópicos).

Uma prova desta proposição pode ser encontrada em [[2] pág 06].

Observação 2.0.7. Se os nós são orientados, isto é, se $S^{1}$ é orientada, a isotopia ambiente deve preservar a orientação.

No caso de enlaçamentos de duas ou mais componentes determinamos uma ordenação fixada para as componentes e exigimos que a isotopia ambiente respeite as ordenações. As classes de equivalência de um nó ou enlaçamento são chamadas de tipo de nó ou tipo de enlaçamento, respectivamente.

Se $K$ é um nó ou enlaçamento em $\mathbb{R}^{3}$, o grupo fundamental $\Pi_{1}\left(\mathbb{R}^{3} \backslash K\right)$ do complemento de $K$ é chamado, simplesmente, o grupo de K. Notemos que o grupo é o mesmo, a menos de isomorfismo, se considerarmos o nó em $S^{3}$ on $\mathrm{cm} \mathbb{R}^{3}$, conforme a proposição abaixo.

Proposição 2.0.8. Se B é qualquer subconjunto limitado de $\mathbb{R}^{n}$ tal que $\mathbb{R}^{n} \backslash B$ é conexo por caminhos e $n \geq 3$, cntão a inclusär induz um isomorfismo

$$
\prod_{1}\left(\mathbb{R}^{n} \backslash B\right) \stackrel{i .}{\longrightarrow} \Pi_{1}\left(S^{n} \backslash B\right)
$$

\section{Demonstraçāo:}

Escolhamos uma vizinhança qualquer $U$ de $\infty$ em $S^{n}$, a qual näo intercepta $B$ e é homeomorfa a $\mathbb{R}^{n}$. Logo, $U \cap \mathbb{R}^{n}=U \backslash \infty \cong S^{n-1}$. Então $U$ e $\mathbb{R}^{n} \cap U$ são simplesmente conexos. Como $S^{n}=U \cup\left(\mathbb{R}^{n} \backslash B\right)$, aplicando o teorema de Seifert-Van Kampen, obtemos o diagrama

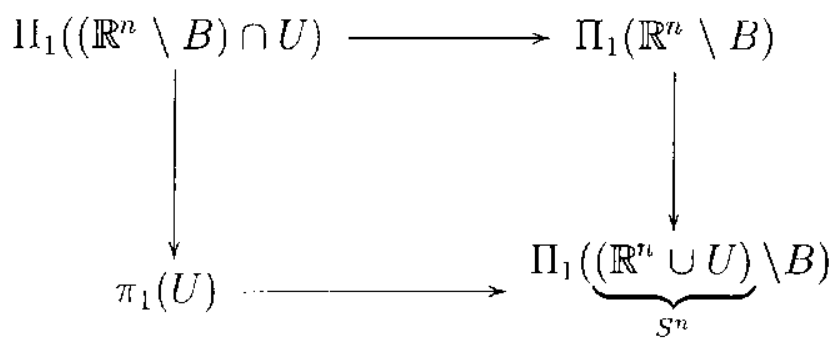

Como $U$ e $\left(\mathbb{R}^{n} \backslash B\right) \cap U=\mathbb{R}^{n} \cap U$ são simplesmente conexos, segue que $\Pi_{1}\left(S^{n} \backslash B\right) \cong$ $\Pi_{1}\left(\mathbb{R}^{n} \backslash B\right)$ 
Definição 2.0.9. A inclusão natural $S^{1} \subset \mathbb{R}^{2} \subset \mathbb{R}^{3} \subset \mathbb{R}^{3} \cup\{\infty\}=S^{3}$ é o nó trivial.

Proposiçāo 2.0.10. O nó trivial tem grupo fundamental cíclico infinito.

\section{Demonstraçāo:}

Seja $P$ o plano $y=0$. Sejam $S_{1}$ e $S_{2}$ os semi-rspaços fechados determinados por $P$.

O nó trivial $K_{0}$ intercepta $S_{1}$ e $S_{2}$ em arcos $A_{1}$ e $A_{2}$ respectivamente. Notemos que $X_{i}=S_{i} \backslash A_{i}, 1 \leq i \leq 2, X_{1} \cap X_{2}$ são abertos e conexos por caminhos e $\mathbb{R}^{3} \backslash K_{0}=X_{1} \cup X_{2}$. Aplicando o teorema de Seifert e Van-Kampen temos que $\Pi_{1}\left(X_{1} \cup X_{2}\right)=\mathrm{II}_{1}\left(\mathbb{R}^{3} \backslash K_{0}\right)=\mathbb{Z}$, desde que $\Pi_{1}\left(X_{i}\right)=\Pi_{1}\left(\mathbb{R}^{3} \backslash\{0\}\right), 1 \leq i \leq 2$.

Definição 2.0.11. Um nó é tame se ele é equivalente a um polígono fechado simples.

No que segue consideraremos apenas nós tames.

\subsection{A Apresentação de Wirtinger.}

Esta seção descreve umı procedimento para escrevermos uma apresentação do grupo de um nó $K$ em $\mathbb{R}^{3}$. Na figura $15, \alpha_{1}, \ldots, \alpha_{n}$ são arcos conexos cuja uniāo é $K$. Consideremos os $\operatorname{arcos} \alpha_{2}, i=1, \ldots, n$ em um plano $P$, digamos o plano $x y$.

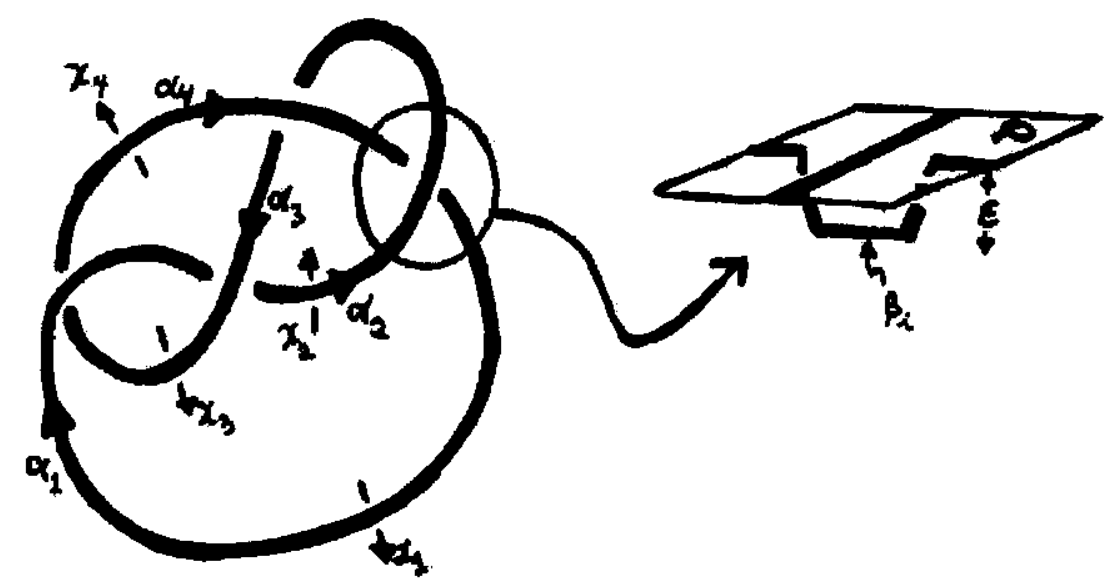

Figura 15

Assumimos uma orientação para o nó. Tracemos uma pequena seta, denotada por $x_{i}$; passando sob cada $\alpha_{i}$ na direção da direita para a esquerda. Então podemos representar um carninho fechado em $\mathbb{R}^{3} \backslash K$ como segue. $O$ ponto $(0,0,1)=*$ é tomado como $o$ 
ponto base (melhor imaginado como o olho do observador). Denotemos também por $x_{i}$ o caminho fechado que consiste no triângulo orientado de $*$ atć o início de $x_{i}$, depois percorrendo $x_{i}$ até o final na direção da seta, e então, retornando a *.

Em cada cruzamento existe uma relação entre os $x_{i}, i=1, \ldots, n$. As duas possibilidades são:

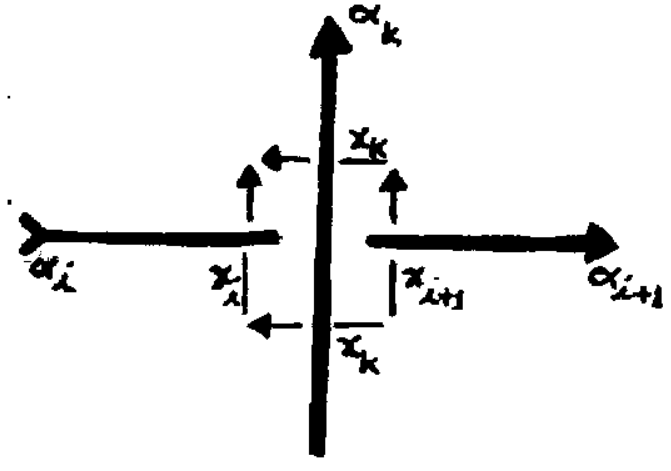

$r_{i}: x_{k} x_{\imath}=x_{i+1} x_{k}$

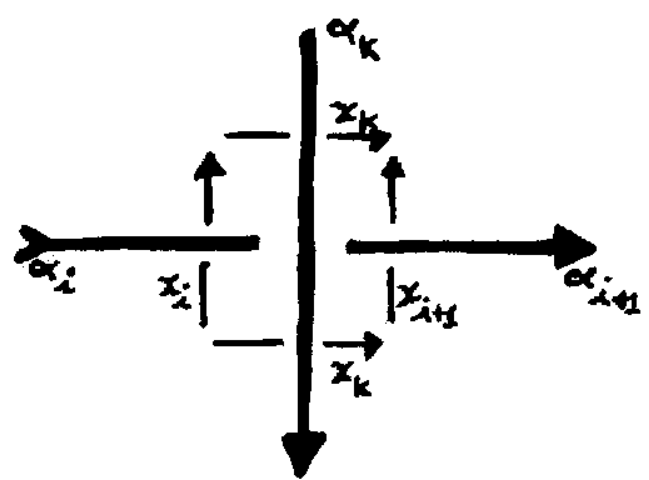

$r_{i}: x_{i} x_{k}=x_{k} x_{i+1}$

Figura 16

Seja $r_{i}$ uma das relaçōes acima. Ao todo existem exatamente $n$ relaçōes $r_{1}, \ldots, r_{n}$, que podem ser encontradas deste modo. Veremos que estas relações formam um conjunto completo de relações.

Teorema 2.1.1. O grupo $\Pi_{1}\left(\mathbb{R}^{3} \backslash K\right)$ é gerado pela classe de homotopia de $x_{i}$ e tem apresentaçâo

$$
\Pi_{1}\left(\mathbb{R}^{3} \backslash K\right)=\left\langle x_{1}, \ldots, x_{n} \mid r_{1}, \ldots, r_{n}\right\rangle
$$

Além disso, qualquer uma das relaçóes $r_{i}$ pode ser omitida e o resultado permanece válido.

\section{Demonstraçāo:}

Lembremos que $K$ está no plano $P=\{z=0\}$ em $\mathbb{R}^{3}$ exceto onde $K$ mergulha para baixo a uma distância $\epsilon$ em cada cruzamento.

Para aplicarmos o teorema de Seifert - Van Kampen, dividiremos $X=\mathbb{R}^{3} \backslash K$ em uma uniäo de $n+2$ partes $A, B_{1}, \ldots, B_{n}$ e $C$. Seja $A=\{z \geq-\epsilon\} \backslash K$. 
O bordo inferior de $A$ é o plano $P^{\prime}=\{z=-\epsilon\}$ com $n$ segmentos de retas $\beta_{1}, \ldots, \beta_{n}$ removidos. Seja $B_{i}^{\prime}$ um paralelepípedo sólido que contém $\beta_{i}$ em seu interior e cuja face superior está em $P^{\prime}$ o juntando um arco que conecta o topo de $B_{i}^{\prime}$ à $*$, não interceptando $K$, obtemos $B_{i}$. Os $B_{i}, i=1, \ldots, n$, podem ser tomados disjuntos dois a dois.

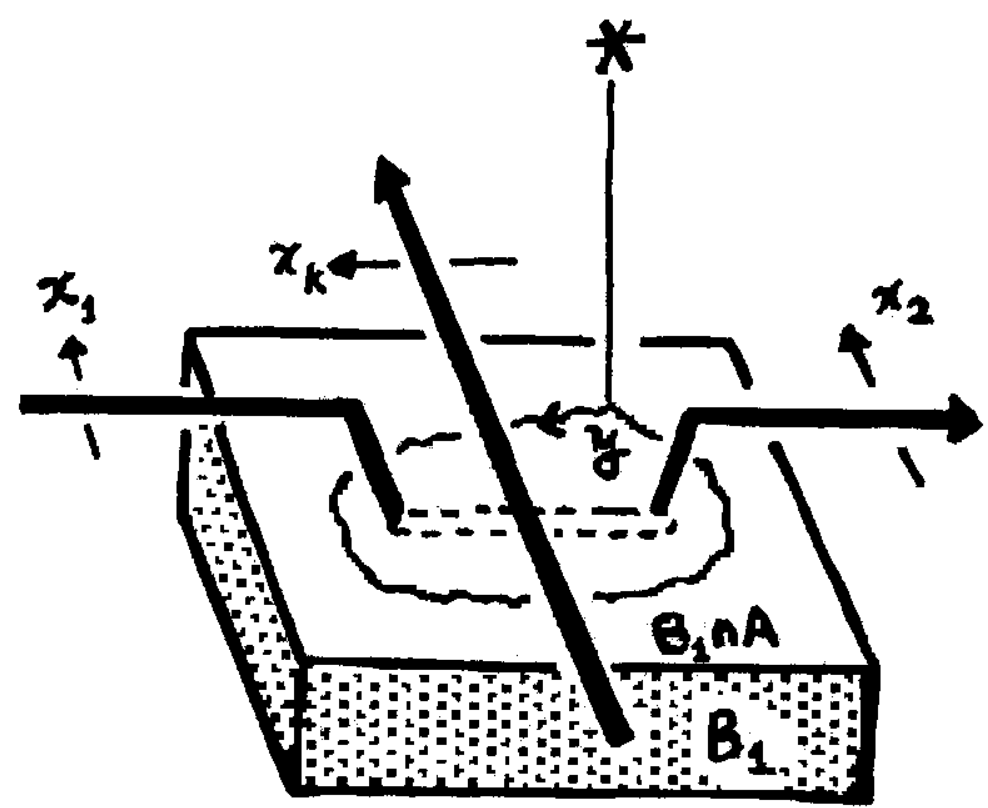

Figura 17

Finalmente, seja $C=$ o fecho de tudo o que cstá abaixo de $A \cup B_{1} \cup \cdots \cup B_{n}$, junto com um arco em $X$ até *.

Desde que o grupo fundamental do nó trivial é $\mathbb{Z}$, segue, por indução, aplicando o teorema de Seifert - Van Kampen, que $\Pi_{1}(A)$ é o grupo livre gcrado por $x_{1}, \ldots, x_{n}$.

Vamos analisar a união de $B_{1}$ e $A$. $B_{1}$ é simplesmente conexo e $B_{1} \cap A$ é um retângulo menos $\beta_{1}$ mais o arco até $*$, então $\Pi_{1}\left(B_{1} \cap A\right)$ é cíclico infinito, com gerador $y$. Como podemos ver na figura $17, y$ como clemento de $A$, torna-se a palavra $x_{k} x_{1}^{-1} x_{k}^{-1} x_{2}$ assumindo um cruzamento do primciro tipo, como na figura 16. Então, temos que

$$
\Pi_{1}(A)=\left\langle x_{1}, \ldots, x_{n} \mid \emptyset\right\rangle, \Pi_{1}\left(B_{1}\right)=\langle\emptyset \mid \emptyset\rangle \text { e } \Pi_{1}\left(A \cap B_{1}\right)=\langle y \mid \emptyset\rangle .
$$

Pelo teorma de Seifert-Van Kampen, $\Gamma_{1}\left(A \cup B_{1}\right)$ tem geradores $x_{1}, \ldots, x_{n}$ e uma única relação $x_{k} x_{1}^{-1} x_{k}^{-1} x_{2}=1$. Esta relação é equivalente a $x_{k} x_{1}=x_{2} x_{k}$, a qual é $r_{1}$. Então

$$
\Pi_{1}\left(A \cup B_{1}\right)=\left\langle x_{1}, \ldots, x_{n}\right| r_{1}>
$$


De modo análogo, adicionando $B_{2}$, obtemos

$$
\Pi_{1}\left(A \cup B_{1} \cup B_{2}\right)=<x_{1}, \ldots, x_{\tau_{\iota}} \mid r_{1}, r_{2}>.
$$

Continuando o processo teremos

$$
\Pi_{1}\left(A \cup B_{1} \cup B_{2} \cup \ldots \cup B_{n}\right)=\left\langle x_{1}, \ldots, x_{n} \mid r_{1}, \ldots, r_{n}\right\rangle
$$

Finalmente, a adição de $C \grave{a} A \cup B_{1} \cup \cdots \cup B_{n}$ não tem efeito no grupo fundamental, desde que $C$ e $C \cap\left(A \cup B_{1} \cup \cdots \cup B_{n}\right)$ são simplesmente conexos.

Isto completa a prova, exceto pela observação de que um dos $r_{i}$, digamos $r_{n}$ é redundante. Para vermos isto, trabalharemos em $S^{3}=\mathbb{R}^{3} \cup\{\infty\}$. Seja $A^{\prime}=A \cup\{\infty\}$ e $C^{\prime}=B_{n} \cup C^{\gamma} \cup\{\infty\}$. Logo: $A^{\prime} \cup B_{1} \cup \cdots \cup B_{n-1} \cup C^{\prime}=S^{3} \backslash K, \Pi_{1}\left(A^{\prime}\right)=\Pi_{1}(A)$, e adicionando $B_{1}, \ldots, B_{n}$ temos o mesmo efeito de antes. Notemos que $C^{\prime} \cap\left(A^{\prime} \cup B_{1} \cup \cdots \cup B_{n-1}\right)$ é simplesmente conexo, sendo a 2-esfera menos um arco, e que $C^{\prime}$ também é simplesmente conexo. Então obtemos a mesma conchusão sem adicionar a relação $r_{n}$.

Exemplo 2.1.2. Vamos calcular o grupo do nó trevo usando o método de Wirtinger.

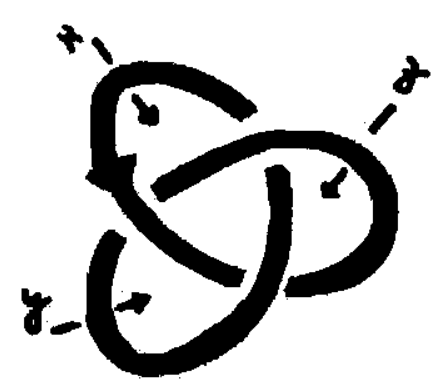

Figura 18

Os geradores são $x, y, z$ e as relaçōes $x z=z y, y x=x z$. A segunda pode ser usada para eliminar $z=x^{-1} y x$, que substituída na primeira relação fornece $y x=x^{-1} y x y$. Assim obtemos a seguinte apresentação para o grupo do nó trevo $\langle x, y \mid x y x=y x y\rangle$, que é a mesma que $<a, b \mid a^{3}=b^{2}>$. De fato, basta considerarmos as seguintes equações: $a=y x, b=y^{2} x, x=a b^{-1} a$ e $y=b a^{-1}$. 


\subsection{O grupo de cobordismo dos nós.}

Definiçāo 2.2.1. Dois enlaçamentos $K_{0}$ e $K_{1}$ em $S^{3}$ sấo ditos cobordantes se existe um mergulho próprio $P L$ (ou diferenciável) $\mathcal{H}$ de $S^{1} \times[0,1] \mathrm{em} S^{3} \times[0,1]$ de modo que os extremos $S^{1} \times\{i\}$ são mergulhados em $S^{3} \times\{i\}$ como $K_{i} \times\{i\}, i=0,1$ e a imagem de $\mathcal{H}$ intercepta $\partial\left(S^{3} \times[0,1]\right)$ transversalmente. Este mergulho é um cobordismo entre $K_{0} e K_{1}$.

Definição 2.2.2. Um nó é dito ser slice se ele é cobordante ao nó trivial.

Definição 2.2.3. Para cada $i \in\{0,1\}$, seja $K_{i}$ um nó orientado em $S_{i}^{3} \cong S^{3}$ e seja $B_{i}^{3}$ uma bola fechada de $S_{i}^{3}$ que intercepta $K_{i}$ em $B_{i}^{1}$, onde $B_{i}^{1}$ é um arco näo enodado. Removendo $B_{i}^{3}$ de $S_{i}^{3}$ e unindo os pares $\left(S_{0}^{3} \backslash B_{0}^{3}, K_{0} \backslash B_{0}^{1}\right)$ e $\left(S_{1}^{3} \backslash B_{1}^{3}, K_{1} \backslash B_{1}^{1}\right)$ através de um homeomorfismo $h:\left(\partial B_{0}^{3}, \partial B_{0}^{1}\right) \rightarrow\left(\partial B_{1}^{3}, \partial B_{1}^{1}\right)$ que reverte orientação obtemos um novo nó orientado em $S^{3}$, chamado a soma conexa dos nós $K_{0}$ e $K_{1}$ e denotado por $K_{0} \# K_{1}$.
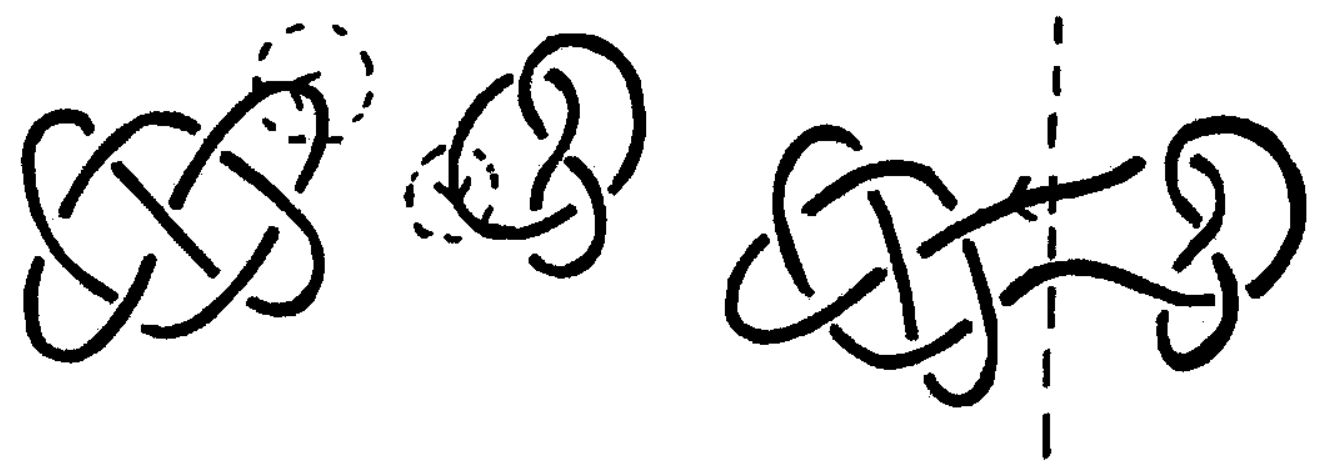

Figura 19

Lema 2.2.4. Se $K$ é um nó orientado qualquer em $S^{3}$ er $: S^{3} \rightarrow S^{3}$ é um homeomorfismo revertendo orientação, então $K \# r K$ é um nó slice.

\section{Demonstração:}

De fato, isto ocorre pois $K \# r K$ pode ser situado em $\mathbb{R}^{3}$ de maneira que $K$ e $r K$ sejam simétricos com relação a um plano, como mostra a figura 21 . A "rotação" de $K$ em torno deste eixo de simetria até $r K$ fornece o disco procurado. 


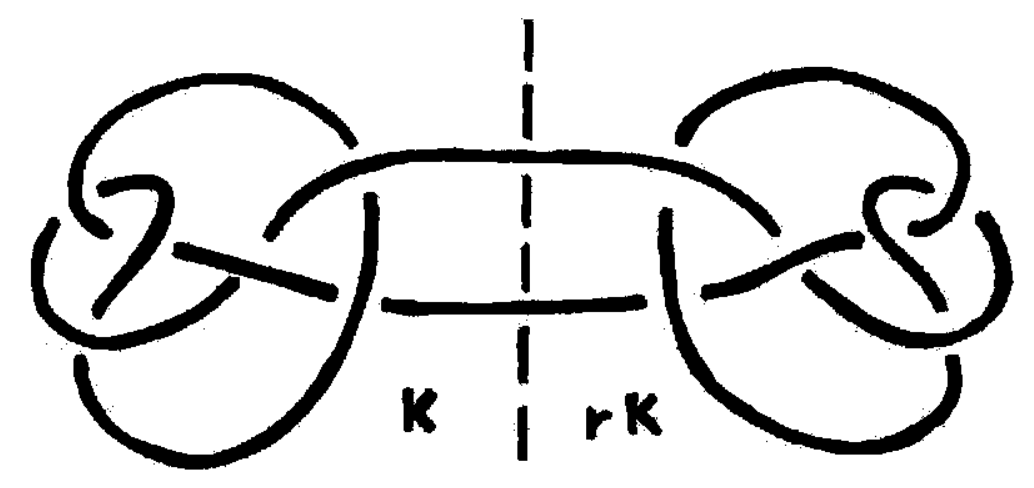

Figura 20

Teorema 2.2.5. O conjunto $\mathcal{C}_{1}$ das classes de cobordismo de nós orientados de $S^{1}$ em $S^{3}$ é urn grupo abeliano com a adiçâo definida por

$$
[J]+[K]=[J \# K]
$$

onde [ ] é uma classe em $\mathcal{C}_{1}$.

\section{Demonstraçāo:}

Veja [[13] pág 228].

Observemos apenas que o elemento neutro é a classe de cobordismo do nó trivial e que o inverso da classe $[K]$ é a classe $[r \bar{K}]$, onde $r: S^{3} \rightarrow S^{3}$ é uma refleção e - denota a orientação oposta.

\subsection{Superfícies de Seifert.}

Definição 2.3.1. Uma superfície de Seifert para um nó ou enlaçamento $K \subset S^{3}$ é uma superfície conexa, compacta e orientável $M \subset S^{3}$ com $\partial M=K$.

Teorema 2.3.2. Todo nó ou enlaçamento em $\mathbb{R}^{3}$ ou $S^{3}$ é o bordo de uma superfície de Seifert.

\section{Demonstração:}

Seja $L$ um enlaçamento em $\mathbb{R}^{3}$. Designamos uma orientação para cada componente de $L$. Consideremos uma projeção regular de $L$ em um plano, isto é, uma projeção cujos 
únicos pontos múltiplos, se houver, são pontos duplos. Próximo de cada cruzamento, apagamos os arcos superior e inferior e os substutuímos por pequenos "atalhos" no plano da projeção como na figura 21 , levando em conta a orientaçāo.

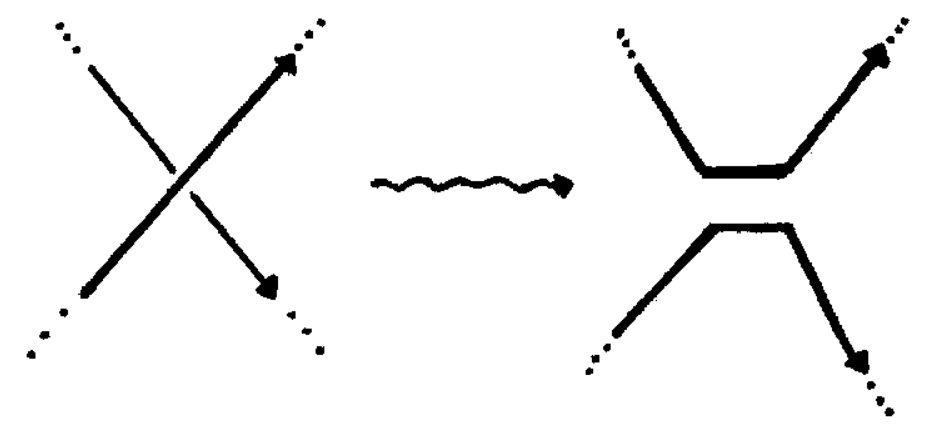

Figura 21

Assim, obtemos uma coleção disjunta de curvas fechadas simples e orientadas no plano. Cada curva é o bordo de um disco no plano, que podem ser feitos disjuntos, empurrando seus interiores ligeiramente para fora do plano, começando com os discos internos e passando para os externos. Além disso, estes discos possuem bicolares, aos quais podem ser associados sinais de $(+)$ e $(-)$ de acordo com a convenção, digamos que o bordo orientado no sentido anti-horário seja visto como o lado positivo. Agora conectemos estes discos nos velhos cruzamentos com retângulos torcidos para obtermos uma superfície $M$ cujo bordo é o enlaçamento $L$.
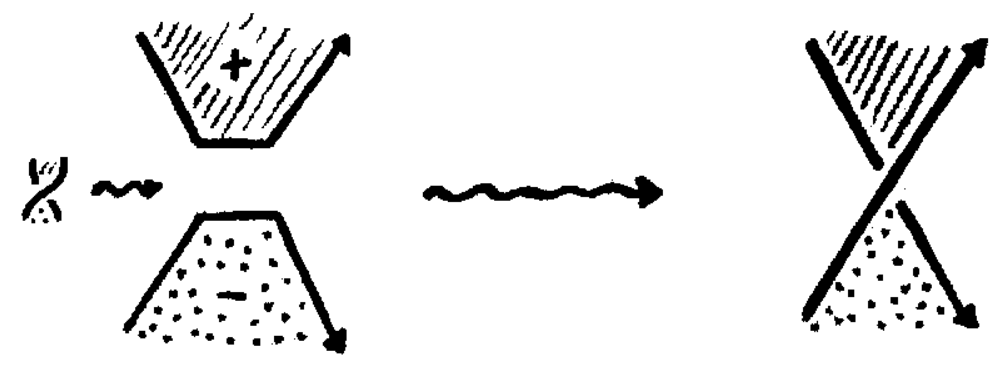

Figura 22

Se $L$ é um nó, $M$ ć concxa. Caso contrário, unimos as componentes por tubos, se necessário.

Exemplo 2.3.3. A figura 23 mostra um enlaçamento e sua superfície de Seifert. 

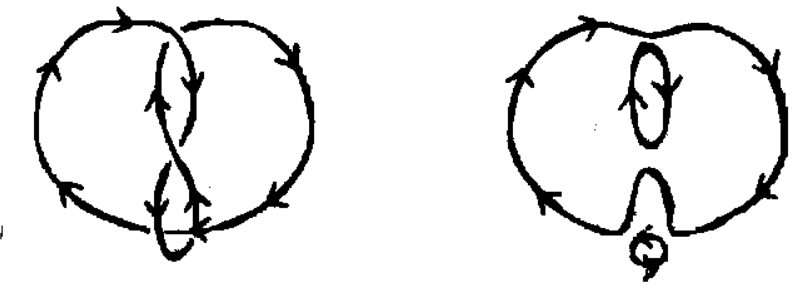

Figura 23a: Eliminar todos os cruzamentos do enlaçamento dado.
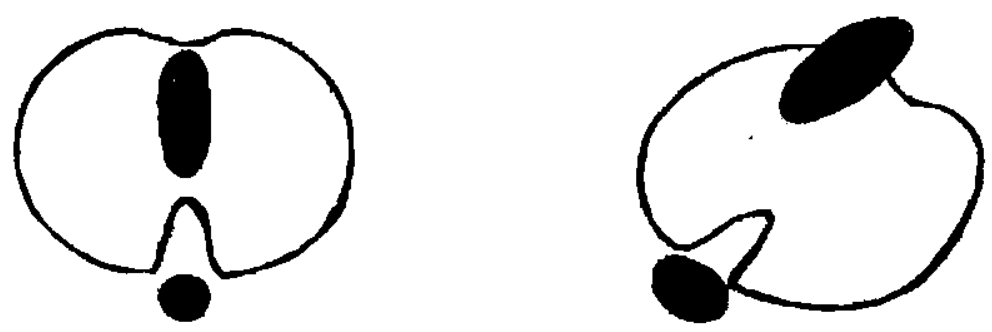

vista lateral

Figura 23b: Os círculos bordam discos em diferentes alturas.

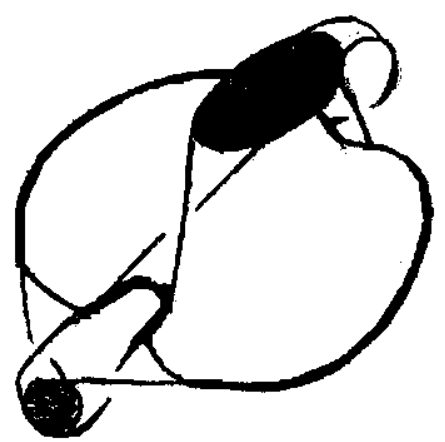

Figura 23c: Conectar os discos através de retângulos torcidos.

Observaçāo 2.3.4. Dependendo da orientação cscolhida para um dado enlaçanento, ó possível obtermos para este mais de una superfícic de Seifort, como vemos nas figuras 21 (.) 25

O enlaçamento dado na figura 25 é conhecido como enlaçamento de Hopf. Neste aso escolhemos a orientaḑào de cada compononte independentemente. 


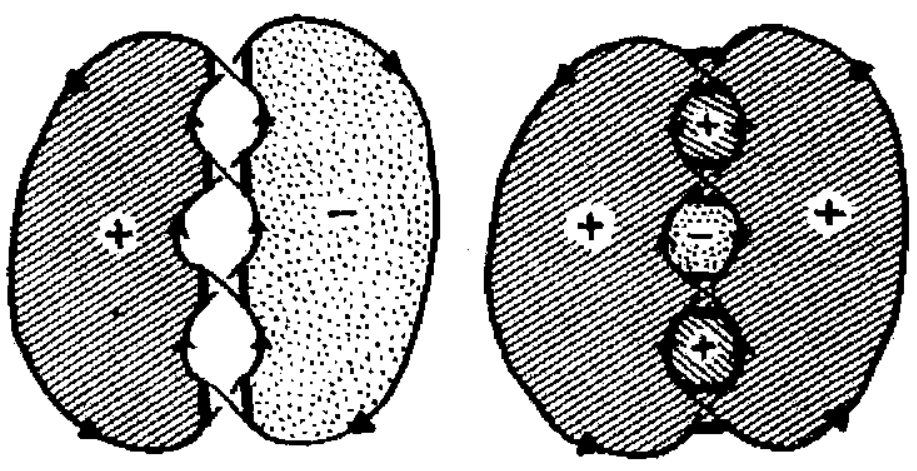

Figura 24

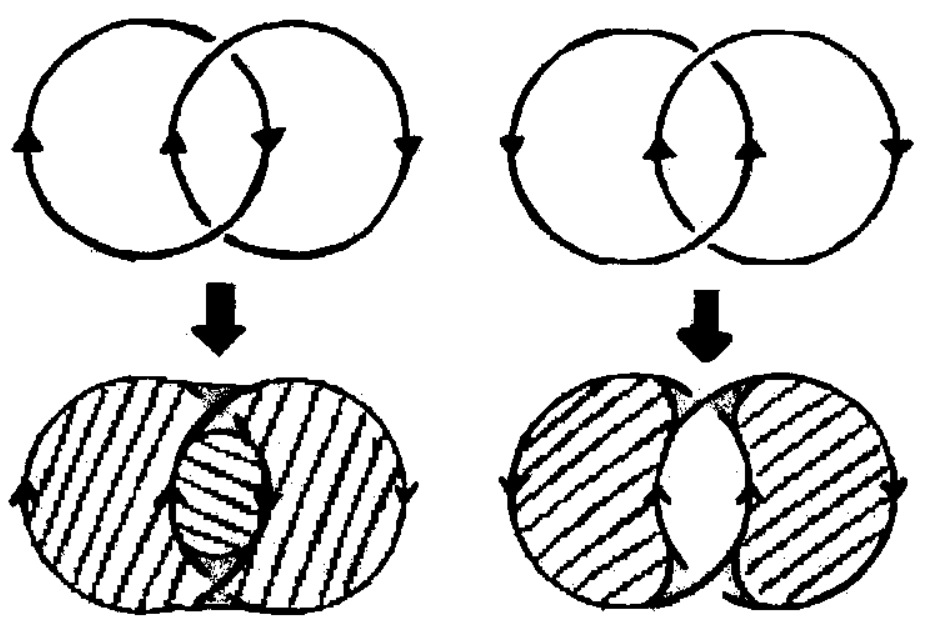

Figura 25

A figura 25 mostra duas escolhas diferentes de orientações para o enlaçamento de Hopf e dias correspondentes superfícies de Seifert.

Observação 2.3.5. Mesmo se a orientação for fixada, a superfície de Seifert de um nó ou enlaçamento não é única. Outras podem ser obtidas adicionando-se alças. Outro exemplo deste fenômeno pode ser visto a seguir. O nó trivial borda o disco padrão, mas pode também ser redesenhado como na figura $26 a$ e visto como o bordo da superfície $26 b$, que consiste em um anel conectado a um pequeno disco por meio de dois retângulos torcidos. 


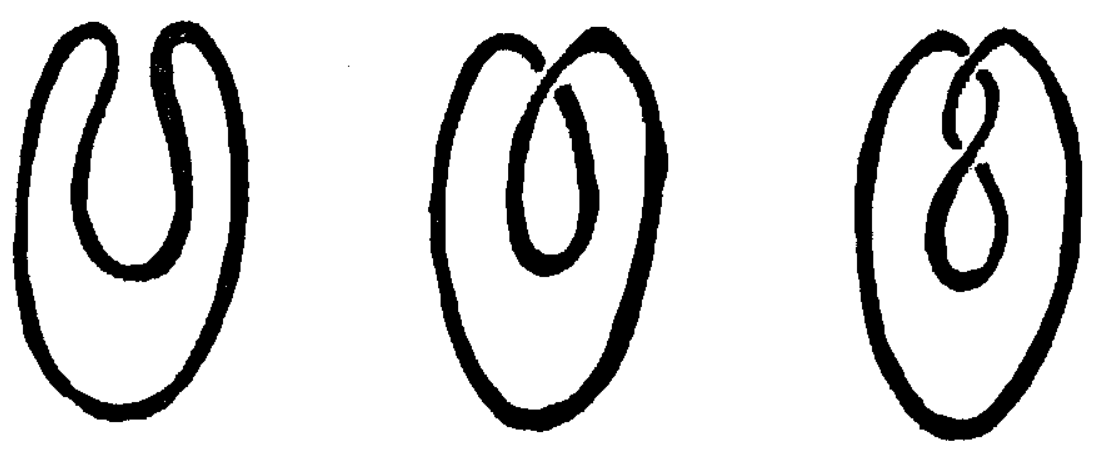

Figura 26a

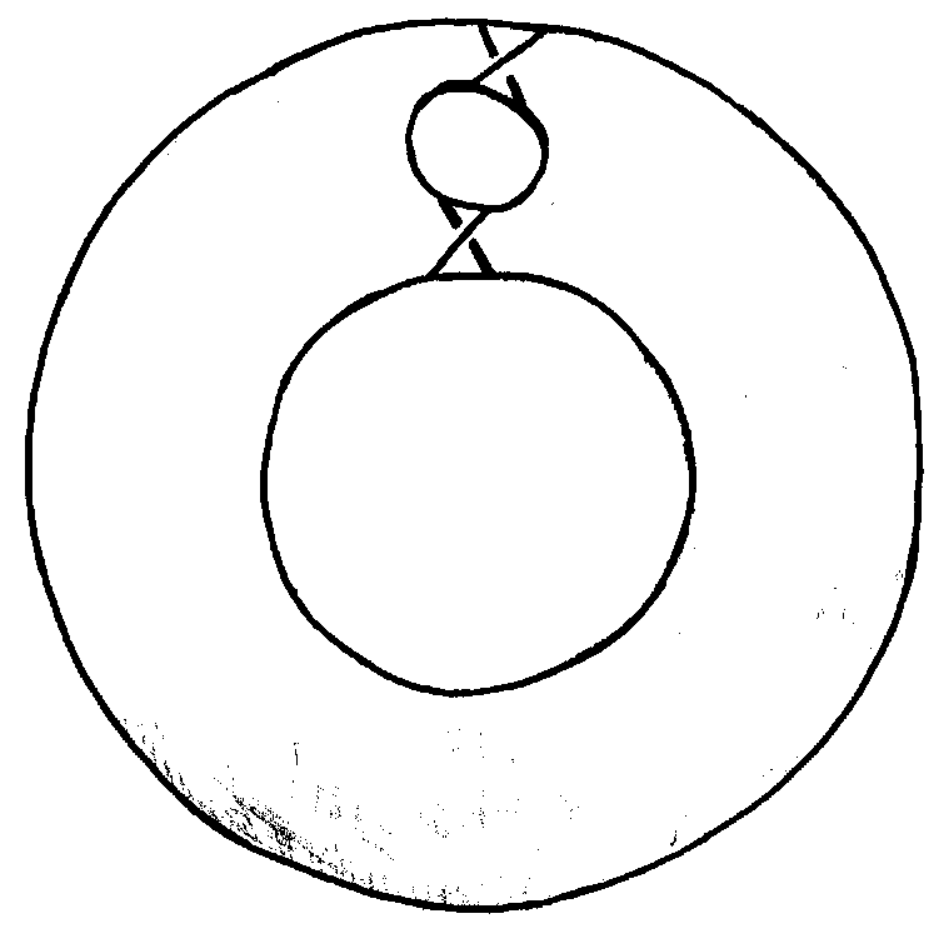

Figura 26b 


\section{Capítulo 3}

\section{Enlaçamentos Bordantes}

\subsection{Enlaçamentos Bordantes.}

Definiçāo 3.1.1. Um enlaçamento bordante $e m S^{3}$ ou em $\mathbb{R}^{3}$ é um enlaçamento cujas componentes bordam superfícies de Seifert disjuntas duas a duas; chamaremos a uniäo de uma tal coleção de superfícies de Seifert, uma para cada componente do enlaçamento, de uma BL-superfície de Seifert para o enlaçamento bordante.

Nesta seção discutiremos métodos para estabelecermos se um enlaçamento é ou não bordante. Primeiro, daremos três definições de número de enlaçamento ("linking number"), as quais são equivalentes (a menos de sinal) pelo Teorema 3.1.3.

Definição 3.1.2. Sejam J e $K$ nós disjuntos orientados em $S^{3}$ ou em $\mathbb{R}^{3}$.

(1) Seja $M$ uma superfície de Seifert para $K$ e seja $N: \operatorname{int} M \times(-1,1) \rightarrow S^{3} u m$ bicolar aberto do interior de $M$, int $M=M \backslash K$, de modo que int $M=N($ int $M \times 0)$. Denotemos $N=N($ int $M \times(-1,1)), N^{+}=($int $M \times(0,1)) e N^{-}=(\operatorname{int} M \times(-1,0))$. Assumimos (modificando $J$ por uma homotopia em $S^{3} \backslash K$, se necessário) que $J$ intercepta $M$ em um número finito de pontos e em cada um destes pontos $J$ passa localmente

(a) de $N^{-}$para $N^{+}$ou

(b) de $N^{+}$para $N^{-}$, segundo as orientações dadas.

Para as interseções do tipo (a) contaremos 1 e para as do tipo (b), -1 . A soma destes valores será denotada por $l k_{1}(J, K)$ e chamada o número de enlaçamento entre $J$ e $K$ (observemos que, a princípio, esta definiçäo parece depender de $M$ ).

(2) Consideremos uma projeçäo reyular de $J \cup K$. Em cada ponto onde J passa sob 
$K$ conta-se -1 ou 1 para os respectivos cruzarnentos

$$
\begin{array}{cc}
-\uparrow \rightarrow \mathbf{J} & -\downarrow \rightarrow \mathbf{J} \\
\mathbf{K} & \mathbf{K}
\end{array}
$$

A soma destes valores, para todos os cruzamentos de $J$ sob $K$, é denotada por $l k_{2}(J, K)$.

(3) Se $J$ é um laço em $S^{3} \backslash K$, então ele representa um elemento de $\Pi_{1}\left(S^{3} \backslash K\right)$ com ponto base apropriado. Este grupo fundamental abelianizado é $\mathbb{Z}$ e o laço $J$ é portanto levado em um número inteiro, denotado por $l k_{3}(J, K)$.

Teorema 3.1.3. $l k_{i}=l k_{j}$ on $l k_{i}=-l k_{j} ; \quad i, j=1,2,3$.

\section{Demonstração:}

Veja [[13] pág 134].

Proposição 3.1.4. Se um enlaşamento $L \subset S^{3}$ ou em $\mathbb{R}^{3}$ possui duas componentes cujo número de enlaçarnento é não-nulo, então $L$ não é um enlaçamento bordante.

\section{Demonstração:}

Utilizemos a definição (1) de número de enlaçamento.

Sejarn $J$ e $K$ duas componentes do enlaçamento $L \subset S^{3}$ cujo número de enlaçamento seja não-nulo e $M$ uma superfície de Seifert para $K$.

Podemos supor que $J$ intercepta $M$ em um número finito de pontos. Vimos que a cada interseção são dados os valores 1 ou -1 . Como, por hipótese, a soma destes valores deve ser diferente de zero, a superfície de Seifert cujo bordo é $J$ intercepta $M$. Então $L$ não é um enlaçamento bordante.

Exemplo 3.1.5. O enlaçamento dado pela figura 27 não é um enlaçamento bordante, pois $l k(J, K)=-3$.

Definição 3.1.6. Um enlaçamento $L=L_{1} \cup \cdots \cup L_{m} \subset \mathbb{R}^{3}$ (ou $S^{3}$ ) com m componentes é splittable se existem $m$ bolas topológicas disjuntas $B_{1}, \ldots, B_{m}$ em $\mathbb{R}^{3}$ (ou $S^{3}$ ) de modo que $L_{i} \subset \operatorname{int}\left(B_{i}\right), i=1, \ldots, m$. 


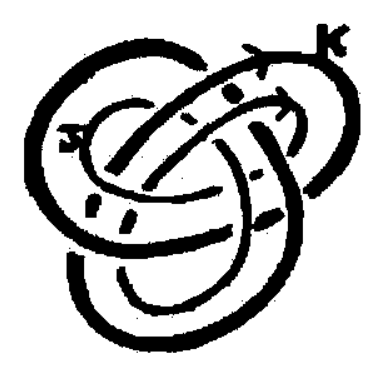

Figura 27

Proposição 3.1.7. Se L é splittable entãa $L$ é um enlaçamento bordante.

\section{Demonstração:}

Assumimos que as componentes $L_{1}, \ldots, L_{m}$ de $L$ pertençam ao interior de bolas disjuntas $B_{1}, \ldots, B_{m}$. Tomomos homeomorfismos $h_{i}: \mathbb{R}^{3} \rightarrow \operatorname{int}\left(B_{i}\right)$, que fixem os pontos de $L_{i}, 1 \leq i \leq m$. Pelo Teorema 2.3.2, cada $L_{i}$ é o bordo de uma superfície de Seifert $M_{i}, 1 \leq i \leq m$, então as superfícies $h_{i}\left(M_{i}\right)$ são novamente superfícies de Scifert para as componentes $L_{i}$, e mais, $h_{i}\left(M_{i}\right)$ são disjuntas, para $1 \leq i \leq m$.

Exemplo 3.1.8. Mostraremos que o enlaçamento dado na figura $28 a$ não é um enlaçamento bordante utilizando um argumento algébrico.

Suponhamos que $J$ seja o bordo de uma superfície de Seifert $M_{J}$ que não intercepta $K$, o que de fato ocorre. Veja um exemplo de $M_{J}$ na figura $28 b$.
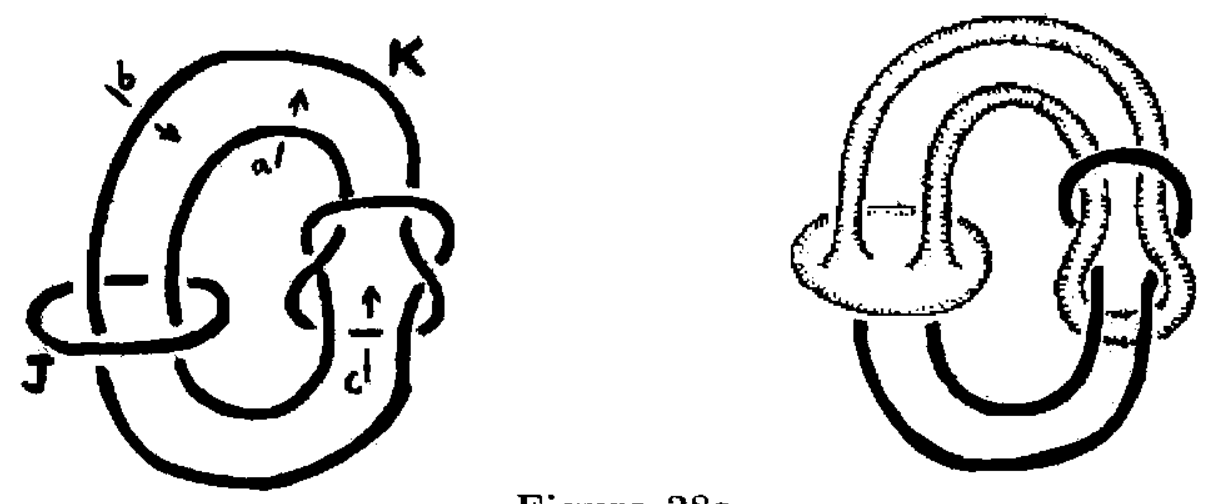

Figura 28a

Figura 28b

A figura $28 \mathrm{c}$ ilustra $M_{J}$ em "abstrato". 


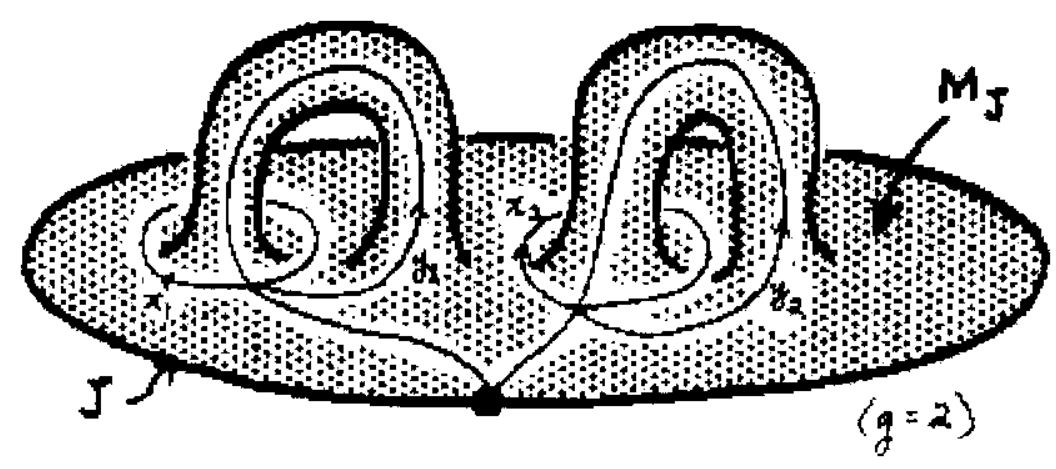

Figura 28c

Notemos que $J$ é homotópico, em $M_{.}$, a um produto de comutadores de certos caminhos fechados em $M_{J}$; a homotopia ocorre na região sombreada, a qual é um disco. A classe de homotopia de $J$ satisfa:

$$
[J]=\left(x_{1} y_{1} x_{1}^{-1} y_{1}^{-1}\right) \cdots\left(x_{g} y_{g} x_{g}^{-1} y_{g}^{-1}\right)
$$

em $\Pi=\Pi_{1}\left(\mathbb{R}^{3} \backslash K\right)$, onde g é o genus de $M_{J}$. Vejamos, por meio das figuras abaixo, que $[J]$ satisfaz a equaçào acima.
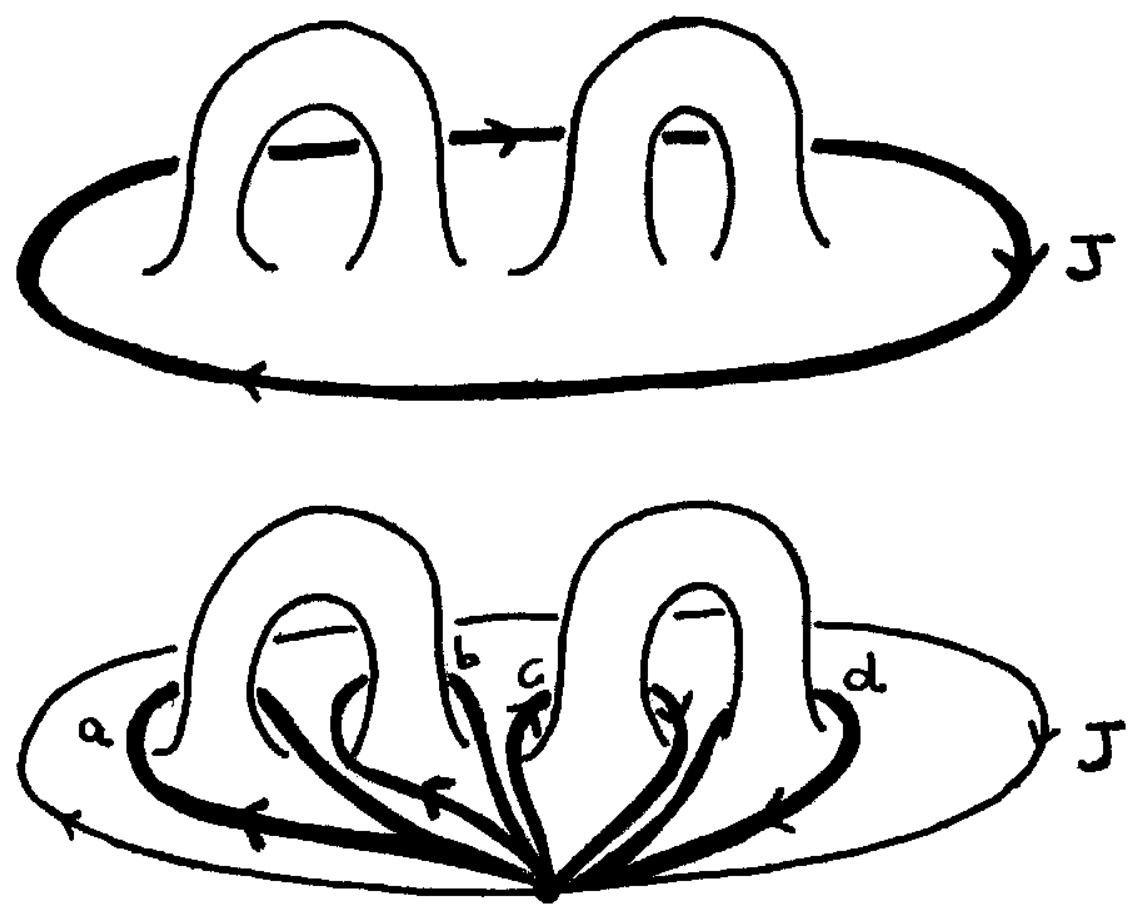
$[J]$ ć homotópico ao produto dos caminhos fechados $a, b, c$ e $d$. Observemos que $a=x_{1}$ e $b=y_{1} x_{1}^{1} \bar{y}_{1}^{-1}$. Analogamente, $c=x_{2}$ e $d=y_{2} x_{2}^{-1} y_{2}^{-1}$.

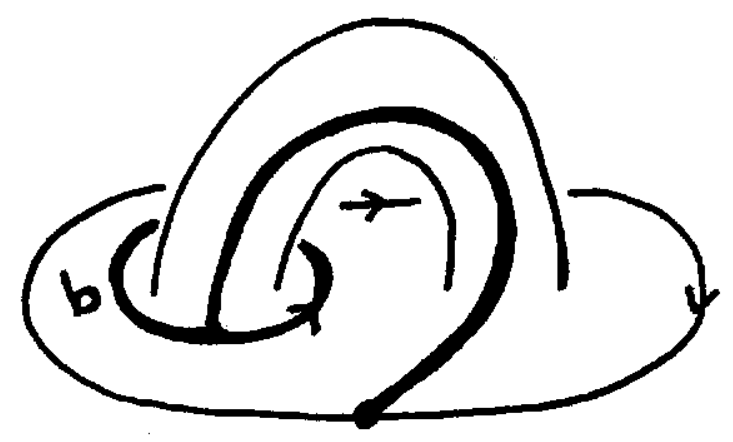

Suponhamos, além disso, que $M_{J}$ é disjunta de uma superfície de Seifert $M_{K}$ para $K$. Então podemos concluir que $l k\left(x_{i}, K\right)=l k\left(y_{i}, K\right)=0$ para todo $i \leq g$ pela Definição 3.1.2. (1) de número de enlaçamento. $\operatorname{Logo}, x_{i}$ e $y_{i}$ estão no subgrupo comutador $C=[\Pi, \Pi]$ de $\Pi=\Pi_{1}\left(\mathbb{R}^{3} \backslash K\right)$ pela Definiçāo 3.1.2. (3). Segue que $[J]$ está no segundo subgrupo comutador de $\mathrm{Il}_{1}\left(\mathbb{R}^{3} \backslash K\right)$, que denotaremos por $C^{t}=[C, C]$. Veremos abaixo que isto não pode ocorrer e csta contradição estabelecerá que as superfícies $M_{J}$ e $M_{K}$ não poden ser disjuntias.

Um cálculo pelo método de Wirtinger fornece uma apresentação $\Pi=\Pi_{1}\left(\mathbb{R}^{3} \backslash K\right)=$ $<a, b, c|a c a=c a c, b c b=c b c\rangle$, onde $a, b$ e $c$ são dados na figura $28 a$.

Seja $S_{3}$ o grupo das permutações de $\{1,2,3\}$. Levando $a, b$ e $c$ nos ciclos (1 2), $\left(\begin{array}{ll}1 & 3\end{array}\right)$ e $\left(\begin{array}{ll}2 & 3\end{array}\right)$, respectivamente, e desde que $\left(\begin{array}{lll}1 & 2\end{array}\right)\left(\begin{array}{lll}2 & 3\end{array}\right)\left(\begin{array}{ll}1 & 2\end{array}\right)=\left(\begin{array}{ll}1 & 3\end{array}\right)=\left(\begin{array}{lll}2 & 3\end{array}\right)\left(\begin{array}{ll}1 & 2\end{array}\right)\left(\begin{array}{ll}2 & 3\end{array}\right)$ e $\left(\begin{array}{ll}1 & 3\end{array}\right)\left(\begin{array}{ll}2 & 3\end{array}\right)\left(\begin{array}{ll}1 & 3\end{array}\right)=\left(\begin{array}{ll}1 & 2\end{array}\right)=\left(\begin{array}{lll}2 & 3\end{array}\right)\left(\begin{array}{ll}1 & 3\end{array}\right)\left(\begin{array}{ll}2 & 3\end{array}\right)$, obtem-se um homomorfismo $h: \Pi \rightarrow S_{3}$. Além disso, $h([J])=h\left(a b^{-1}\right)=\left(\begin{array}{lll}1 & 2\end{array}\right)\left(\begin{array}{ll}1 & 3\end{array}\right)=\left(\begin{array}{lll}1 & 2 & 3\end{array}\right)$. Como qualquer homomorfismo leva os segundos comutadores nos segundos comutadores, concluímos que $[J] \notin[C, C]$ pela seguinte observação.

Observação 3.1.9. O subgrupo comutador $C=\left[\begin{array}{l}S_{3}, S_{3} \\ \text { O }\end{array}=\left\{\left(\begin{array}{lll}1\end{array}\right),\left(\begin{array}{lll}1 & 2 & 3\end{array}\right),\left(\begin{array}{lll}1 & 3 & 2\end{array}\right)\right\}\right.$ é cíclico com gerador $\left(\begin{array}{lll}1 & 2 & 3\end{array}\right)$; o segundo subgrupo comutador $[C, C]$ consiste apenas do elemento identidade.

A figura 29a é um exemplo de enlaçamento bordante e sua BL-superfície de Seifert é dada em $29 b$. 


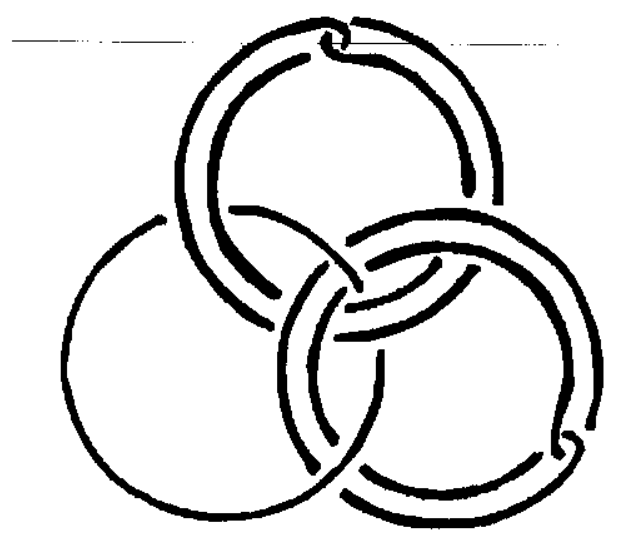

Figura 29a

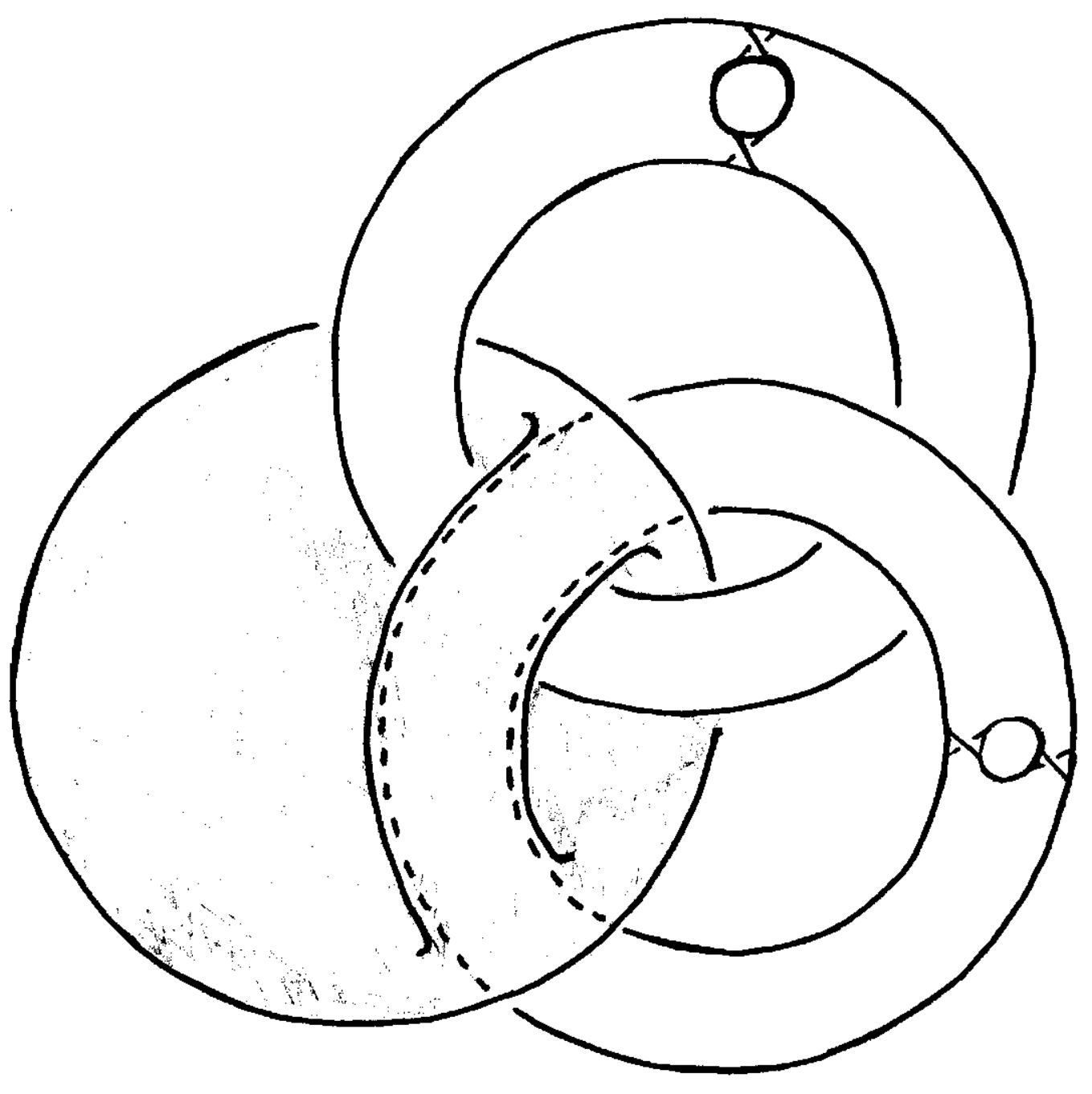

Figura 29b 
Definição 3.1.10. Seja $T(K)$ uma vizinhança tubular de um nó $K \subset S^{3}$. Uma curva fechada simples $\mathbf{m}$ em $\partial T(K)$ a qual é homotópica a zero em $T(K)$ e não é homotópica a. zero em $\partial T(K)$ é chamada um meridiano de $K$.

Seja $L \subseteq S^{3}$ um enlaçamento, e $T$ uma vizinhanç̧a tubular para $L, X(L)$ o fecho de $S^{3} \backslash T$, o qual será chamado de exterior de $L$, e $m \subset \partial T \subset X(L)$ um meridiano da i-ésima componente de $L$.

Conectando-se m a um ponto base de $X-X(L)$ obtem-se um elemento de $I_{1}(X)$, o qual fica bem definido a menos de conjugação, e que será também chamado um i-ćsimo meridiano de $L$.

Seja $F(k)$ o grupo livre gerado por $x_{1}, x_{2}, \ldots, x_{k}$.

Proposição 3.1.11. L é um k-cnlaçamento bordante em $S^{3}$ se, e somente se, existe um epimorfismo $\eta: \mathrm{I}_{1}(X) \rightarrow F(k)$ o qual leva um i-ésimo meridiano para $x_{i}$ para $i=1,2, \ldots, k$.

\section{Demonstração:}

Sejam $M$ uma BL-superficie de Seifert para $L, \bar{M}=M \cap X$ e $S_{1}, \ldots, S_{k}$ as componentes de $\bar{M}$. Desde que $\bar{M}$ é uma união disjunta de superfícies conexas limitadas, $X \backslash \bar{M}$ é conexo por caminhos.

Como as $S_{i}, 1 \leq i \leq k$, possuem bicolares, existem abertos $N_{i}=$ int $S_{i} \times(0,1)$ disjuntos dois a dois.

$X$ pode ser aplicado em umla rosácea de $k$ folhas $\bigvee_{i=1}^{k} S^{1}$, enviando-se os pontos fora de $\bigcup_{i=1}^{k} N_{i}$ no vértice e um ponto de $S_{i}$ x $\{t\}$ no ponto $t$ do $i$-ésimo círculo, o qual está parametrizado pelo intervalo $[0,1]$. A aplicação induzida no grupo fundamental é o epimorfismo requerido.

Reciprocamonte, seja $\mu: F(k) \rightarrow \Pi_{1}(X)$ um homomorfismo que leva $x_{i}$ ao $i$-ésimo meridiano, cuja imagem pela $\eta$ é $x_{i}$, para cada $i \in\{1, \ldots, k\}$.

Seja $\bigcup_{i-1}^{k} S^{1}$ o espaço consistindo de $m$ cópias de $S^{1}$ disjuntas duas a duas. Especificando pontos $p_{1}, p_{2}, \ldots, p_{k}$ um em cada cópia de $S^{1}$ em $\bigcup_{i-1}^{k} S^{1}$, obtemos uma aplicação de um conjunto de $k$ pontos em $\bigcup_{i=1}^{k} S^{1}$ 
Seja $B$ o espaço cone desta aplicação. Realizamos $\mu$ por um mergulho $i: B \rightarrow X$. Seja $e: B \rightarrow \bigcup_{i=1}^{k} S^{\prime}$ a aplicação que leva os arcos de $B$ que ligam o ponto cone às $S^{1}$ no ponto base de $\bigcup_{i=1}^{k} S^{1}$, de modo que $e_{*}=i d_{k(k)}$.

Desde que $i_{*} \cdots \mu, e_{*}=i d_{F(k)}=\eta \mu$, existe uma extensäo $f: X \rightarrow \bigcup_{i=1}^{k} S^{1}$ tal que $f i=e$ e $f_{*}=\eta$

As imagens inversas por meio de $f$ de $k$ pontos distintos, um de cada círculo de $\bigcup_{i=1}^{k} S^{3}$, e distintos do vértice, fornece $k$ superfícies de Seifert disjuntas $M_{1}, \ldots, M_{k}$. Notemos que descle que a restriçào $\left.f\right|_{\partial X}$ comporta-se como a aplicação que projeta a $i$-ésima cópia de $S^{1} \times S^{1}$ de $\bigcup_{i-1}^{k}\left(S^{1} \times S^{1}\right)$ na i-ésima $S^{1}$ de $\bigvee_{i-1}^{k} S^{1}$, então a $i$-ésima cópia de $\partial X$ contém somente $\partial M_{i}$. Desprezando as componentes fechadas de $M=M_{1} \cup \cdots \cup M_{k}$, se houver, obtemos uma BL-superfície de Seifert para $L$ adicionando colares a $M$.

Definição 3.1.12. Um epimorfismo $\eta: \Pi_{1}(X) \rightarrow F(k)$, como na proposição acima, que leva um $i$-ésimo metidiano no gerador $x_{i}$ de $F(k)$, para $i=1,2, \ldots, k$, será chamado de aplicação splitting ( $/ u$ cisão) para $L$.

Exemplo 3.1.13. O enlaçamento trivial $L_{0}$, o qual é bordante tem grupo fundamental livre em dois geradores (como se vê utilizando o teorema de Seifert Van-Kampen ou a apresentação de Wirtinger), gerado pelos seus meridianos, logo existe splitting $\eta: \Pi_{1}\left(L_{0}\right) \rightarrow F^{\prime}(x, y)$.
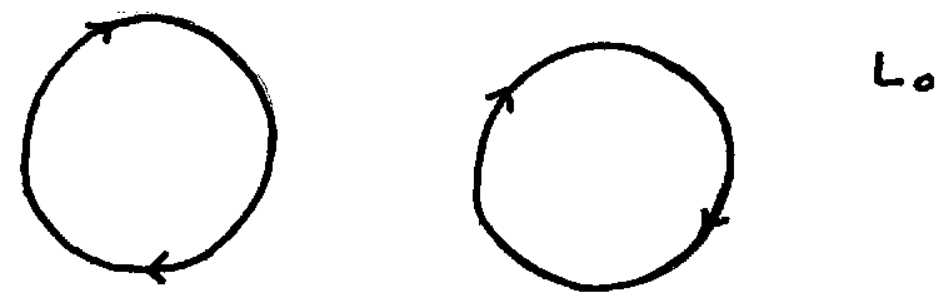

Já o enlaçamento $L_{1}$ da figura abaixo tem grupo fundamental $\mathbb{Z} \oplus \mathbb{Z}$ e portanto não existe epimorfismo $\eta: \Pi_{1}\left(L_{1}\right) \rightarrow F(x, y), \log 0 L_{1}$ não é bordante.

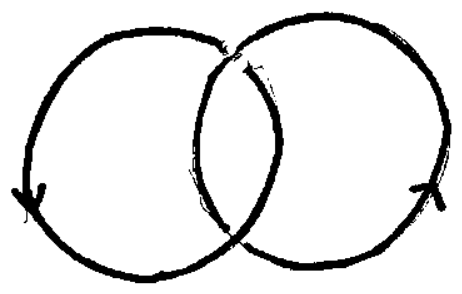




\subsection{Enlaçamentos $F(k)$.}

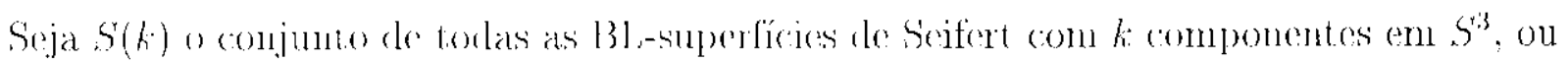
sejal, $M \in S(l)$ se : somente se,

(i) $M$ c mua unià ordenada disjunta de superficies conexas e orientadas $M_{1}, M_{2}, \ldots, M_{k}$ ('mI $\mathrm{S}^{\prime \prime \prime}$.

(ii) Para loclo i. o M, ó homeomorfo a $S^{1}$.

A crientagăo ale $M$ determina um campo de vetores normais de $M \mathrm{~cm} S^{3}$ chamado de direção normal positiva de $M$, do librado normal trivial de $M$ em $S^{3}$, e vice-versa.

1)(finmos a seguinte relacào no conjunto $S(k)$. Sejam $M, N$ em $S(k) . M$ a $N$ são cobordantes relativamente ao bordo ("boundary (obordant") se existe uma subvariedade $\mathcal{I M}$ de $S^{63} \times I$ de modo clu:

(i) $\operatorname{IM} \cap\left(S^{3} \times 0\right)-M 0 \mathcal{I M} \cap\left(s^{3} \mathrm{x} 1\right)=\mathrm{N}:$

(ii) $\partial \mathcal{I} \mathcal{M}-m+(M \cup N)$ i homeomorfo a $k$ copian de $S^{1} \times I$

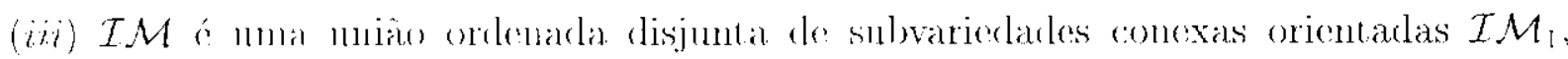

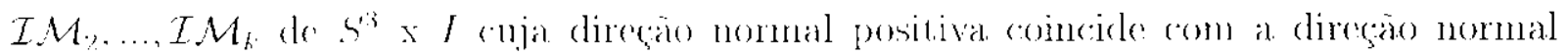
positiva de $M$ (m) $S^{3} \times 0$ o de $N$ ems $S^{3} \times 1$.

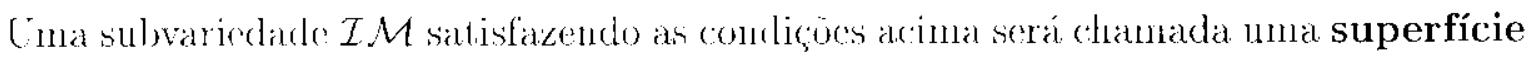
de Seifert de um cobordismo de cnlaçamentos bordantes, ou simplesmente um cobordismo bordante. $\partial \mathcal{I} M-i m(M J N)$ ichamale um cobordismo de enlaçamentos

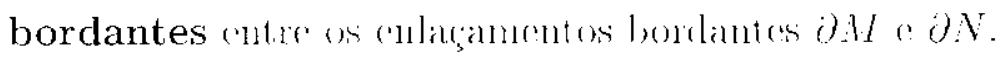

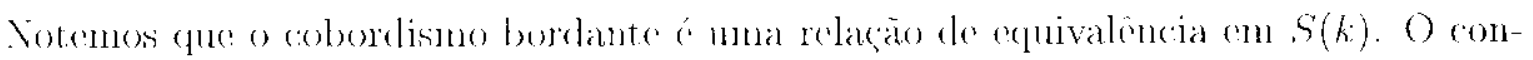

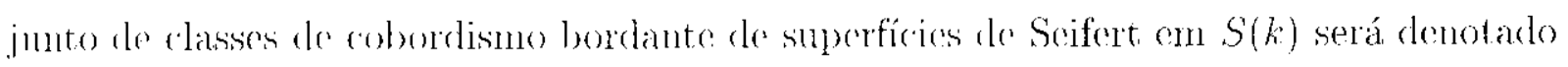
jol $C\left(B_{k}\right)$.

Definição 3.2.1. Um. $F(k)$-cnlaçamento ‘ um par $(L, \eta)$. onde L é um ki-enlaçamento

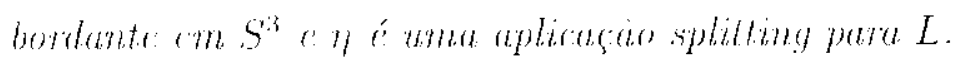

Sejann $L_{i}, i=0,1$, culacancutos com exteriores $X_{i}=X\left(L_{i}\right), i=0,1$ o $\mathcal{L} \mathcal{L}$ a imagem

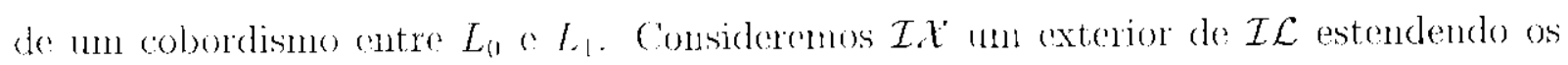

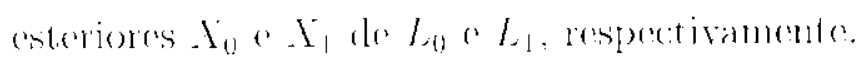

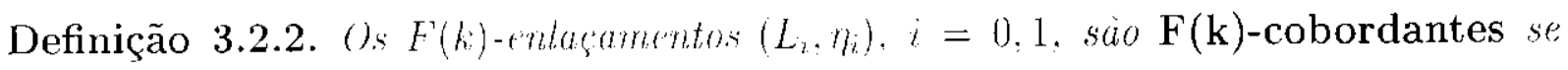

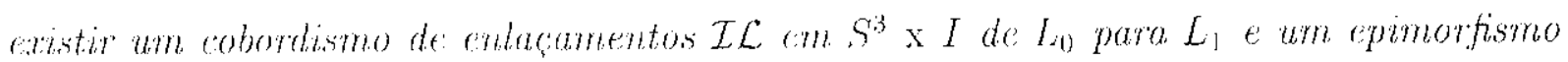


$\Theta: \mathrm{I}_{1}(\mathcal{I X}), F(k)$ tal que $\eta_{i}-\Theta \cdot j_{i}$. a memos de: andomorfismos internos de $F(k)$, para

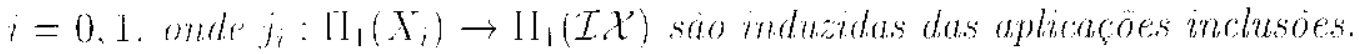

Observação 3.2.3. Suponhamos qur $f \cdot g: A \rightarrow B$ sejan epimorfismos de grupos. Fintäo fe $g$ diferem por um antomonfismo intomo de A se s somente so eles diferem por um antomorfisno interno de $B$. Togo, na definicão acima de $F(k)$-cobordismo a ambiguidade na escolha de pontos bases de $X_{0} . X_{1}$ e $\mathcal{I X} 6$ absorvida pelos automorfismos internos de $F(k)$.

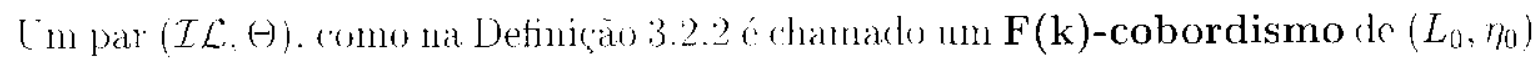
para $\left(L_{1} \cdot \eta_{1}\right)$. Denotaremos por $C_{1}\left(F^{\prime}(k)\right)$ o conjunto das classes de $F(k)$-cobordismo de Feth-enlagenamentos.

Lema 3.2.4. Stga IL um cobordismo de enlaçamento entre os enlaçamentos bordantes

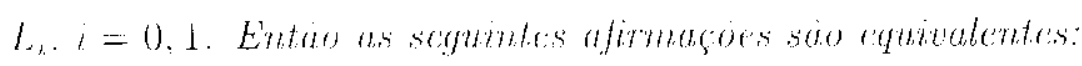

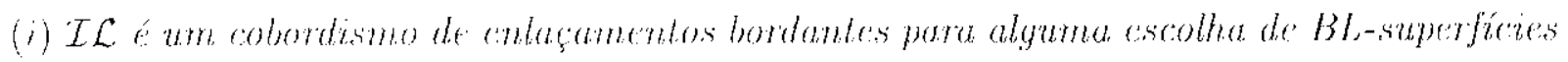
de Seifert de: $L_{0}$ : $L_{1}$.

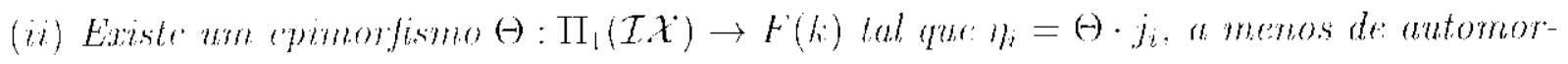

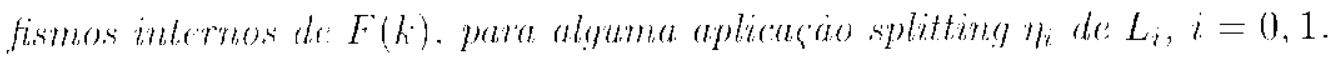

\section{Demonstração:}

A demonstraça r análoga à da Proposiçāo 3.1.11. utilizando a Observação 3.2.3. loja [10|. pág 6633].

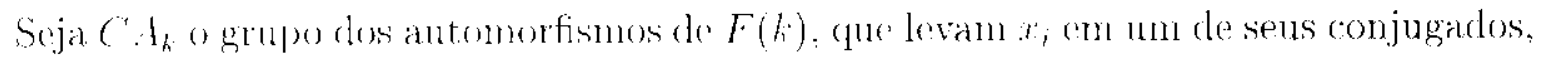
palal (ada $;-1, \ldots .$.

Votemos (que se $\eta_{1}$ e $\eta_{2}$ săo aplicaçós splitting para un k-onlaçamento l, em $S^{3}$, entào $\eta_{2}=\alpha \cdot \eta_{1}$, para algum $a \in C A_{k}$ on seja. quaiscuer duas aplicaçoes split.ing para um $L$ diferem por un autonortismo de $F(k)$ (uue leva $x_{i}$ em um de seus conjugados, $1 \leq i \leq k$.

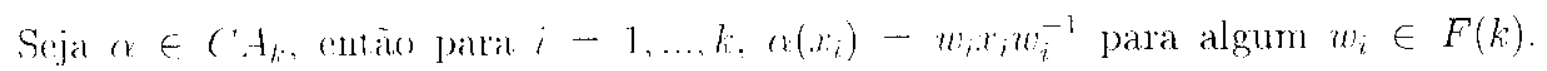

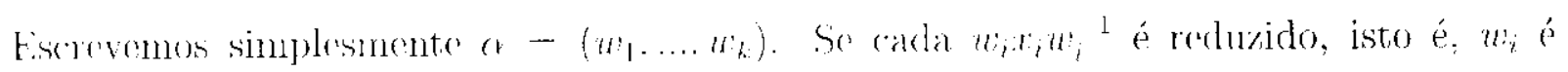

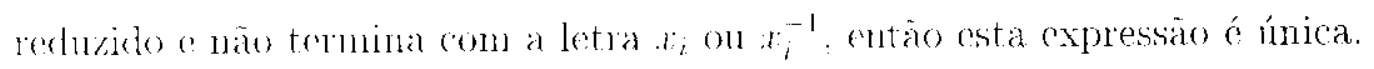

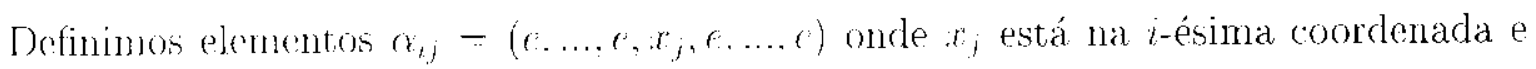

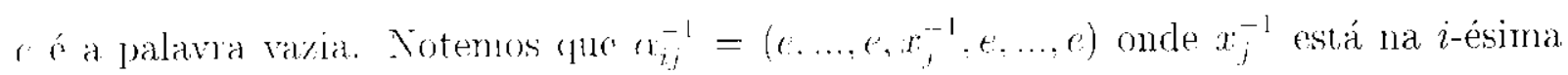
coordenadia. 
Lema 3.2.5. C.1. égerado por crijpara $i, j \quad 1 \ldots k$ a $i+j$

\section{Demonstração:}

$C A_{k}$ é de fato, un grupo pois se a $x-\left(w_{1}, \ldots, w_{k}\right)$ entadu $\alpha^{-1}=\left(\alpha^{-1}\left(w_{1}^{-1}\right), \ldots\right.$,

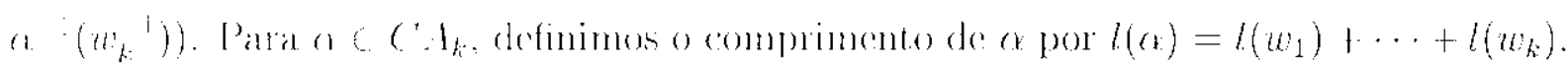

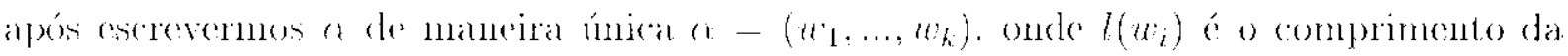

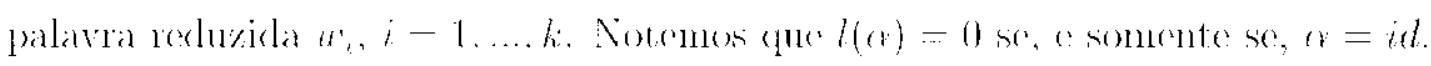

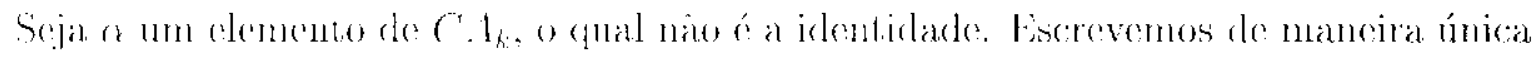

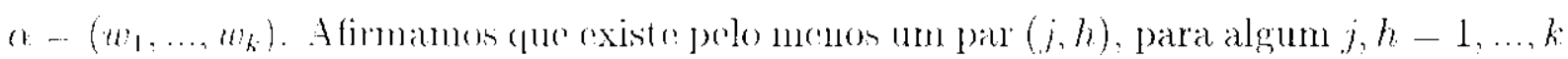

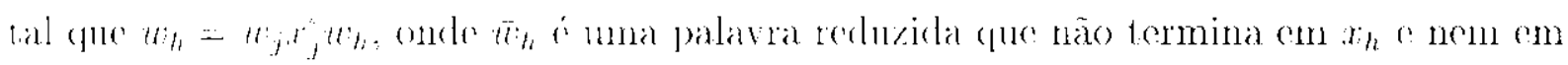

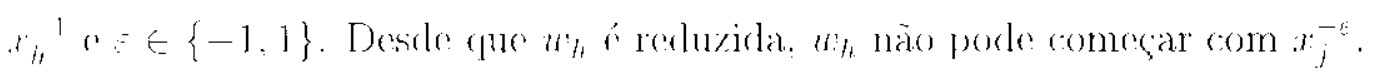

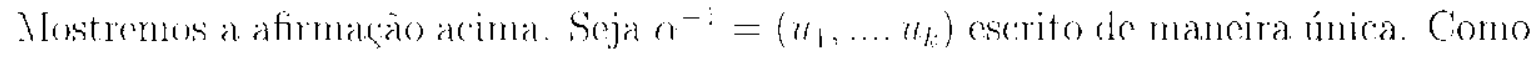
supomos que of $\neq$ id. existe um $h-1, \ldots, k$ tal que wh não é a palavra vazia e também

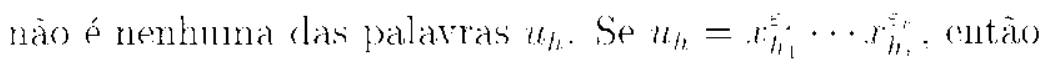

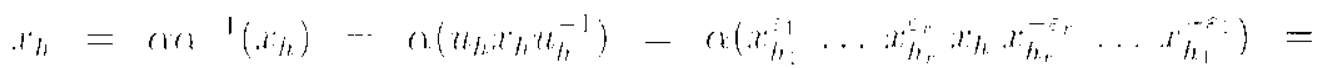

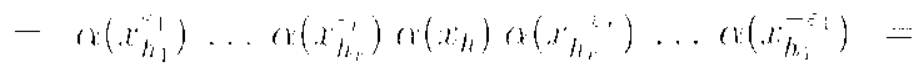

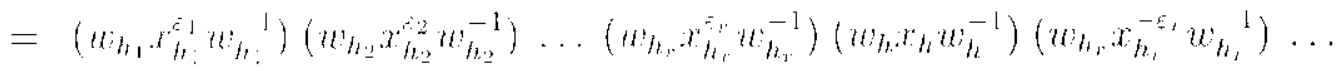

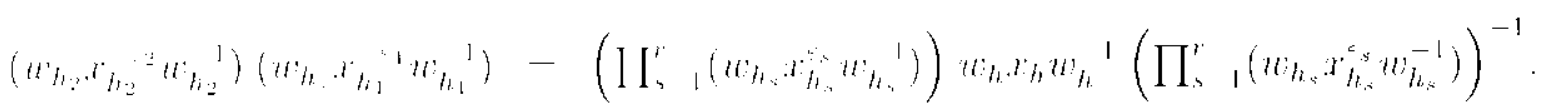

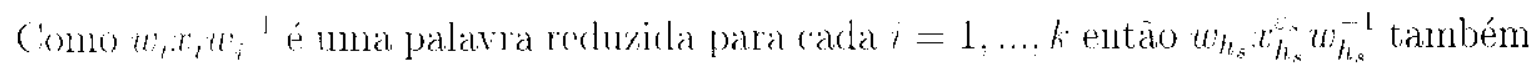

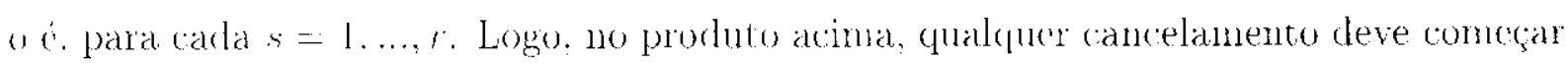

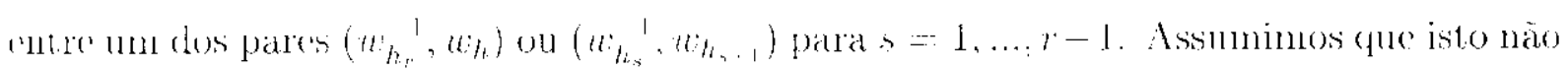

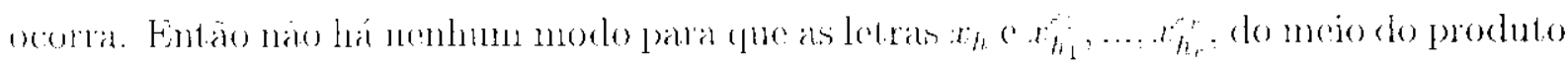

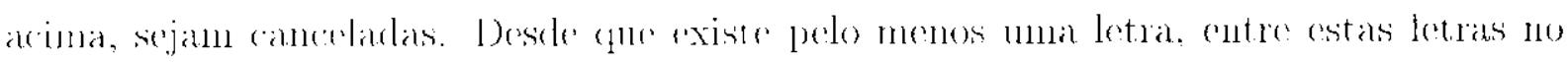

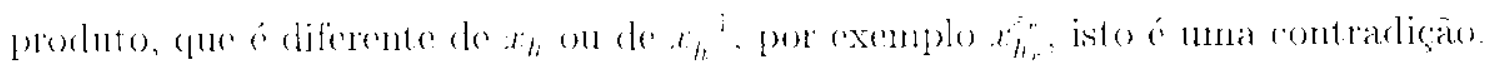

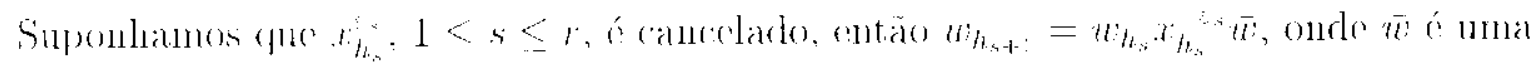

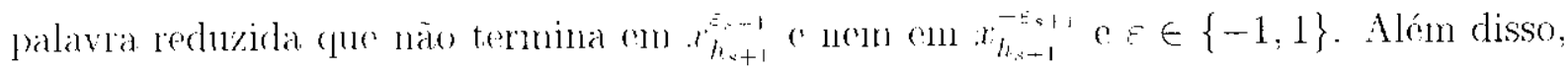

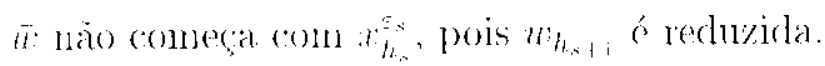

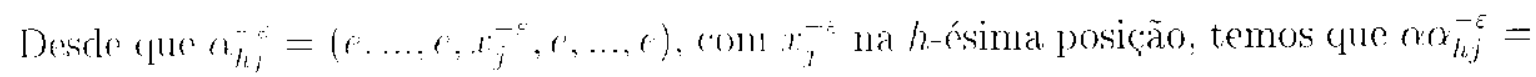

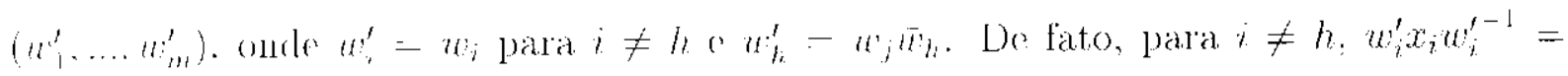

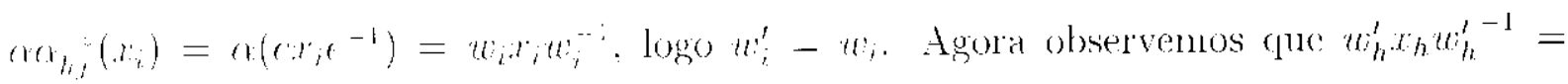




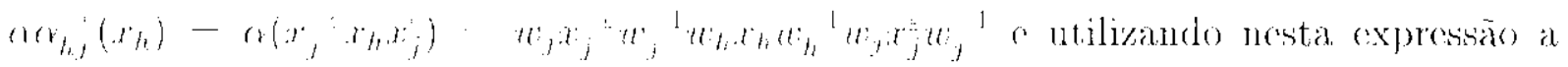

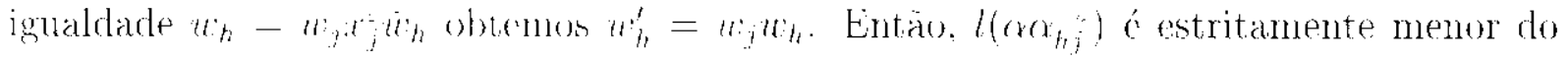

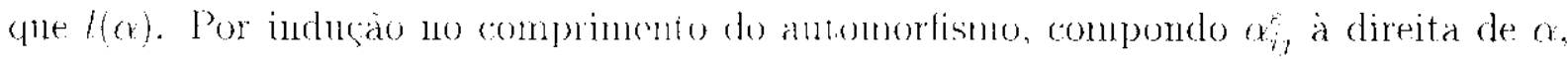
oblemes a identidake. 


\section{Capítulo 4}

\section{Enlaçamentos de Intervalos}

\subsection{O Grupo $C_{1, k}$.}

Nestia seção introduriremos o conjunto $C_{1, k}$ das classes de cobordismo dos enlaçamentos de $k$ intervalos e veremos que ele possui uma estrutura natural de grupo.

Notações: I denota o intervalo fechado $[0,1]$ e $D$ o disco unitário em $\mathbb{R}^{3}$. Para todo inteiro $k>0$, o conjunto $\{1,2, \ldots, k\}$ será denotado por $\underline{k}$ e $a_{1}, a_{2}, \ldots, a_{k}$ serâo $k$ pontos distintos e alinhados pertencentes ao interior do disco unitário $D$. Designaremos por $j_{0}: \underline{k} \times I \rightarrow D \times I$ a aplicação definida por $j_{0}(i, x)=\left(a_{i}, x\right) \in D \times I, i=1,2, \ldots, k$.

Definiçāo 4.1.1. Utm enlaçamento de $k$ intervalos ou um k-enlaçamento de intervalos, $k \geq 1$, é um mergulho próprio PL (ou diferenciável) localmente plano e transversal ao bordo $f: \underline{k} \times I \rightarrow D \times I$ tal que $\left.f\right|_{\underline{k} \times \partial(I)}=\left.j_{0}\right|_{\underline{k} \times \partial(I) .}$

O enlaçamento $j_{0}$ é chamado de enlaçamento trivial.

$\mathrm{Na}$ figura 30 pode ser visto um exemplo de um 3-enlaçamento de intervalos.
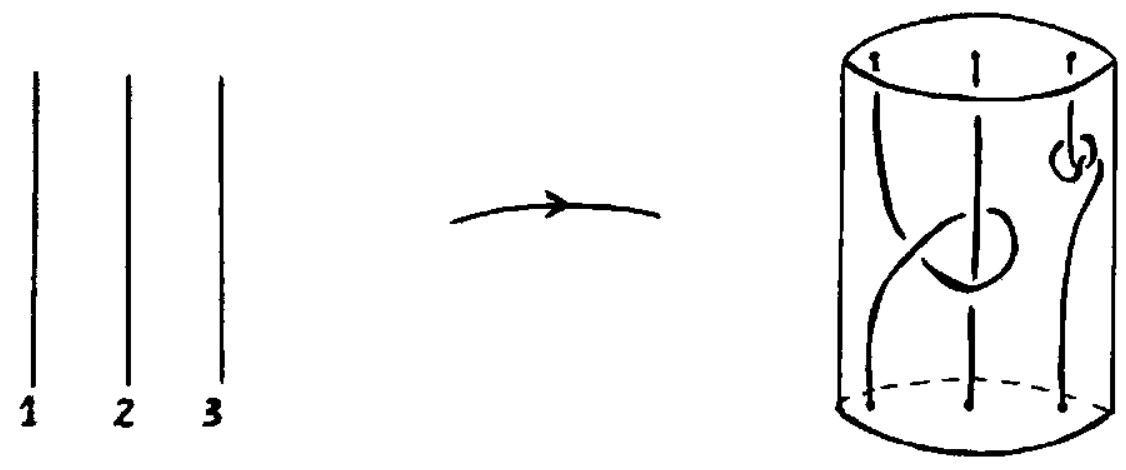

Figura 30 
Definição 4.1.2. Dois k-enlaçamento de intervalos $f, g: \underline{k} \times I \rightarrow D \times I$ são cobordantes se existe um mergulho próprio PL (ou diferenciável) localmente plano e transversal ao bordo $F^{\prime}: \underline{k} \times I \times I \rightarrow D \times I \times I$ tal que

$$
\left.F\right|_{\underline{k} \times I \times 0}=f,\left.F\right|_{\underline{k} \times I \times 1}=\left.g e F^{\prime}\right|_{\underline{k} \times \partial(I) \times I}=\left(\left.j_{0}\right|_{\underline{k} \times \partial(I)}\right) \times i d_{I} .
$$

Vamos definir o produto de dois $k$-enlaçamento de intervalos. Para isso, consideremos as aplicaçōes $\alpha_{1}, \alpha_{2}: D \times I \rightarrow D \times I$ definidas por $\alpha_{1}(y, t)=(y, t / 2), \alpha_{2}(y, t)=(y,(1+$ t) $/ 2),(y, t) \in D \times I$.

Definiçāo 4.1.3. Se $f_{1}$ e $f_{2}$ säo $k$-cnlaçamenlos de intervalos, seu produto $f_{1} \cdot f_{2}$ : $\underline{k} \times I \rightarrow D \times I$ é o k-enlaçamento de intervalos definido por

$$
f_{1} \cdot f_{2}(i, l)= \begin{cases}\alpha_{1} f_{1}(i, 2 t) & \text { se } 0 \leq t \leq 1 / 2 \\ \alpha_{2} f_{2}(i, 2 t-1) & \text { se } 1 / 2 \leq t \leq 1\end{cases}
$$

Observemos que para todo $k$-enlaçamento de intervalos $f$, temos que $f \cdot j_{0} ; j_{0} \cdot f$ e $f$ são cobordantes, na verdade, são isotópicos. Logo, no conjunto $C_{1, k}$, o enlaçamento $j_{0}$ é o elemento identidade.

O próximo resultado nos mostra que $f^{-1}$ definida abaixo é o enlaçamento inverso de $f$.

Sejarn $\alpha: D \times I \rightarrow D \times I$ uma aplicação definida por $\alpha(y, t)=(y, 1-t)$ e $f$ um $k$ enlaçamento de intervalos. Definimos $f^{-1}: \underline{k} \times I \rightarrow D \times I$ como o seguinte $k$-enlaçamento de intervalos:

$$
f^{-1}(i, t)=\alpha f(i, 1-t)
$$

Proposição 4.1.4. Para todo k-enlaçamento de intervalos $f, f \cdot f^{-1}$ é cobordante a $j_{0}$.

\section{Demonstraçāo:}

Seja $h: \partial(I \times I) \rightarrow \partial(I \times I)$ um homeomorfismo tal que $h(0 \times I)=0 \times I \cup I \times 0 \cup$ $1 \times I, h(0,1)=(0,1), h(0,0)=(1,1)$. A aplicação $h$ prolonga-se radialmente a um homeomorfismo $h: I \times I \rightarrow I \times I$.

Seja $f: \underline{k} \times I \rightarrow D \times I$ um $k$-enlaçamento de intervalos. Definimos $F: \underline{k} \times I \times I \rightarrow$ $D \times I \times I$ por 

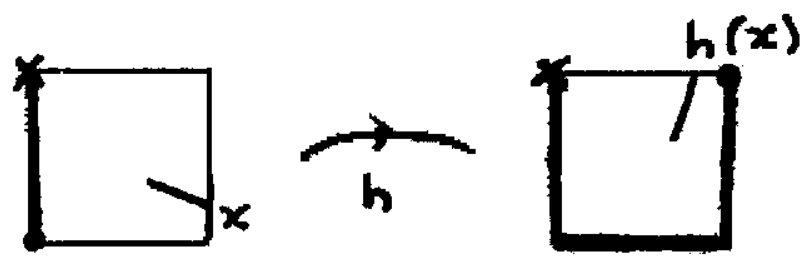

Figura 31

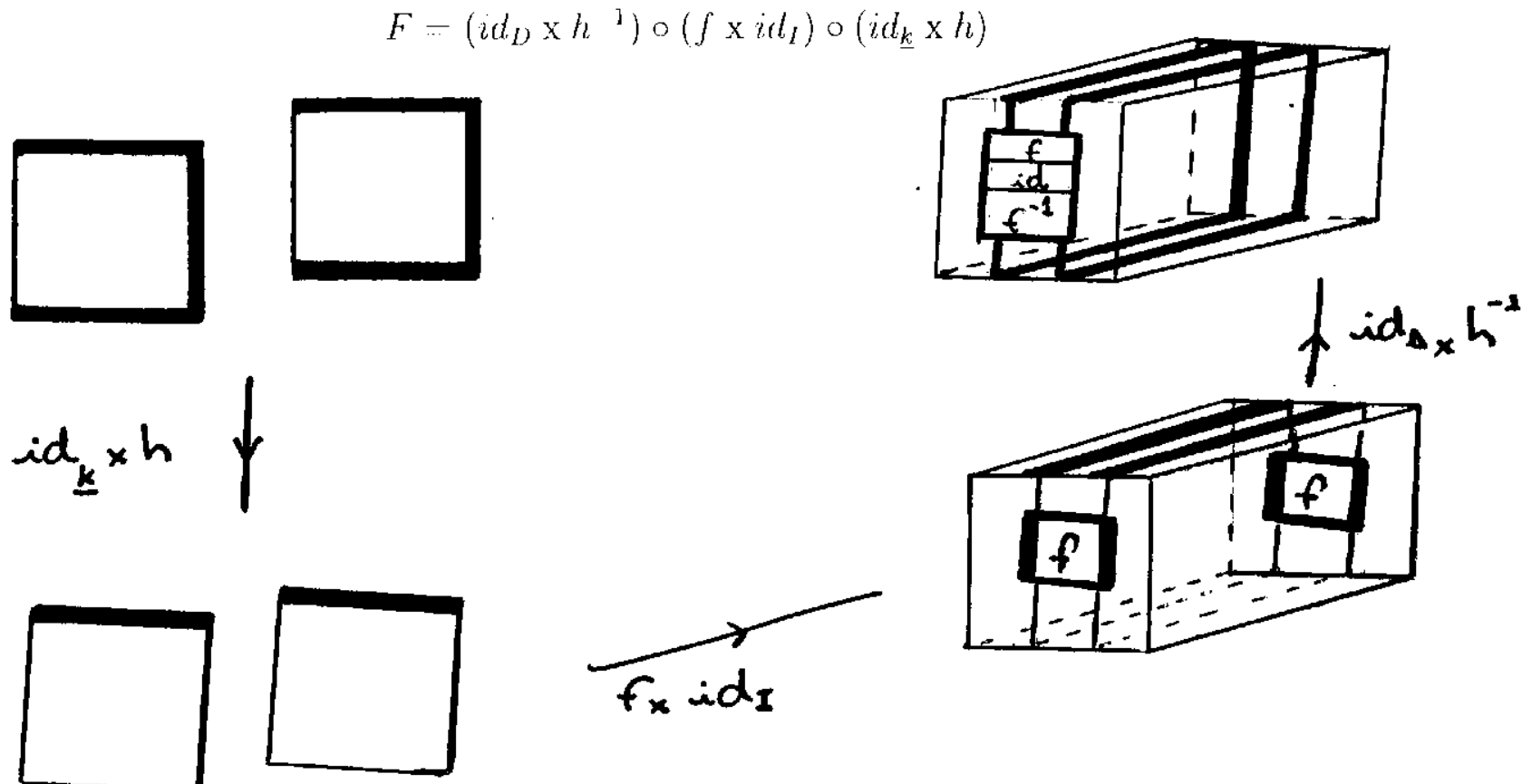

Figura 32

Então $\left.F\right|_{\underline{k} \times I \times 0}=j_{0}$ e $\left.F\right|_{\underline{k} \times I \times 1}=f \cdot j_{0} \cdot f^{-1}$. Portanto, $f \cdot f^{-1}$ é cobordante a. $j_{0}$.

Teorema 4.1.5. As classes de cobordismo dos k-enlaçamentos de intervalos formam um grupo, que será denotado por $C_{1, k}$.

Um k-enlaçamento de intervalos estende-se de maneira única, a menos de isotopia, a um mergulho $\vec{f}: \underline{k} \times I \times D \rightarrow D \times I$.

Seja $F: \underline{k} \times D \times I \rightarrow D \times I \times I$ um cobordismo cntre $k$-enlaçamentos de intervalos $f$ e g. F prolonga-se a $\bar{F}: \underline{k} \times D \times I \times D \rightarrow D \times I \times I$.

Definição 4.1.6. O exterior de um $k$-enlaçamento de intervalos é o fecho de $D \mathrm{x}$ $I \backslash \bar{f}(\underline{k} \times I \times D)$ e será denotado por $X(f)$. 
Analogamente, ") exterior de um cobordismo entre $k$-enlaçamentos de intervalos $X(F)$ é o fecho de $D \times I \times I \backslash \bar{F}(\underline{k} \times D \times I \times D)$.

\subsection{A Ação de Habegger-Lin e o Cobordismo d-baseado.}

Vamos definir uma ação do grupo $C_{1,2 k}$ no conjunto $C_{1, k}$. Para isso seja $B$ o espaço identificaça de $D \times I$, onde identificamos $(z, 0) \operatorname{com}(z, 1)$, para todo $z \in D$ e realizamos as identificações ao longo de $\partial(D)$ x $I$ como segue. Seja $z=(x, y)$ com $x \geq 0, y \geq 0$ e $x^{2}+y^{2}=1$, identificamos $(z, t) \operatorname{com}(z, 1 / 4-t(\bmod 1))$ para $t \in[0,1]$. Então $B$ é uma bola de dimensão três que pode ser decomposta em três subbolas $B_{+}, B_{0}$ e $B_{-}$, as quais correspondem ao produto de $D$ com os intervalos $[0,1 / 4],[1 / 4,1 / 2] \cup[3 / 4,1]$ e $[1 / 2,3 / 4]$ respectivamente.

$B_{0}$ é o produto de um par de discos unitários $D$ colados ao longo do seguinte arco do bordo $\left\{(x, y) / x \geq 0, y \leq 0, x^{2}+y^{2}-1\right\}$, com um intervalo $I$, $\operatorname{logo}$ pode ser identificado com $D \times I$, com $D$ tendo $2 k$ pontos distinguidos. Os $k$ pontos em $B_{0} \subseteq B$ que correspondem a $\left(a_{i}, 0\right) \in D \times I$, serão denotados por $p_{2}$. Os pontos $\left(a_{i}, 3 / 4\right),\left(a_{i}, 1 / 4\right) e\left(a_{i}, 1 / 2\right)$ serão denotados, respectivamente, por $p_{i}^{\prime}, \tilde{p}_{i}$ e $\tilde{p}_{i}^{\prime}$.
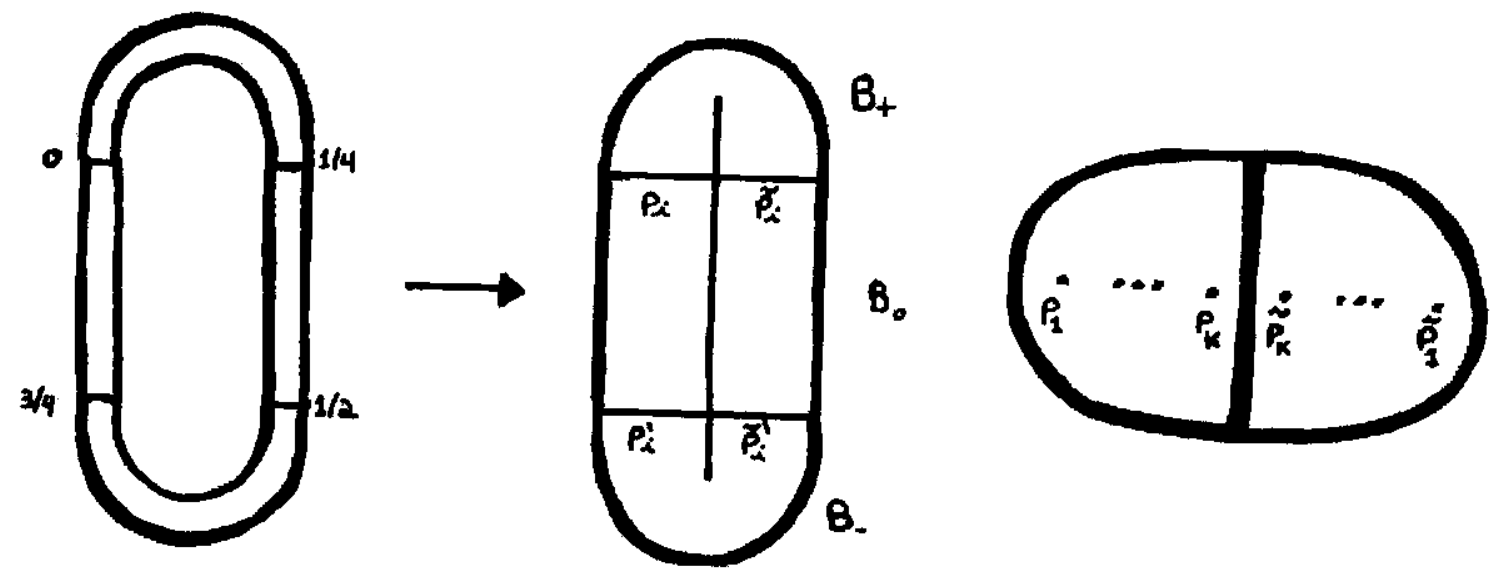

Figura 33

Podemos considerar um $2 k$-enlaçamento de intervalos como sendo um mergulho

$$
\beta: 2 \underline{k} \times I \rightarrow B_{0}
$$


tal que para todo $i \in \underline{k}, \beta(i, 0)=p_{i} \in D \times\{0\}, \beta(i, 1)=p_{i}^{\prime} \in D \times\{3 / 4\}, \beta(k+i, 0)-$ $\tilde{p}_{k-i+1} \in D \times\{1 / 4\} \odot \beta(k+i, 1)=\tilde{p}_{k-\imath+1}^{\prime} \in D \times\{1 / 2\}$.

Um k-enlaçamento de intervalos $f$ pode ser considerado como um mergulho $f: \underline{k} \times I \rightarrow B$. de modo que para todo $i \in \underline{k}, f(i, 0)=p_{i}^{\prime} \in D \times\{3 / 4\}$ \& $f(i, 1)=\tilde{p}_{i}^{\prime} \in$ $D \times\{1 / 2\}$.

$\Lambda$ "uniäo" de $f$ e $\beta$ nos dá um mergulho $\underline{k} \times 1 \rightarrow B_{0} \cup B$.

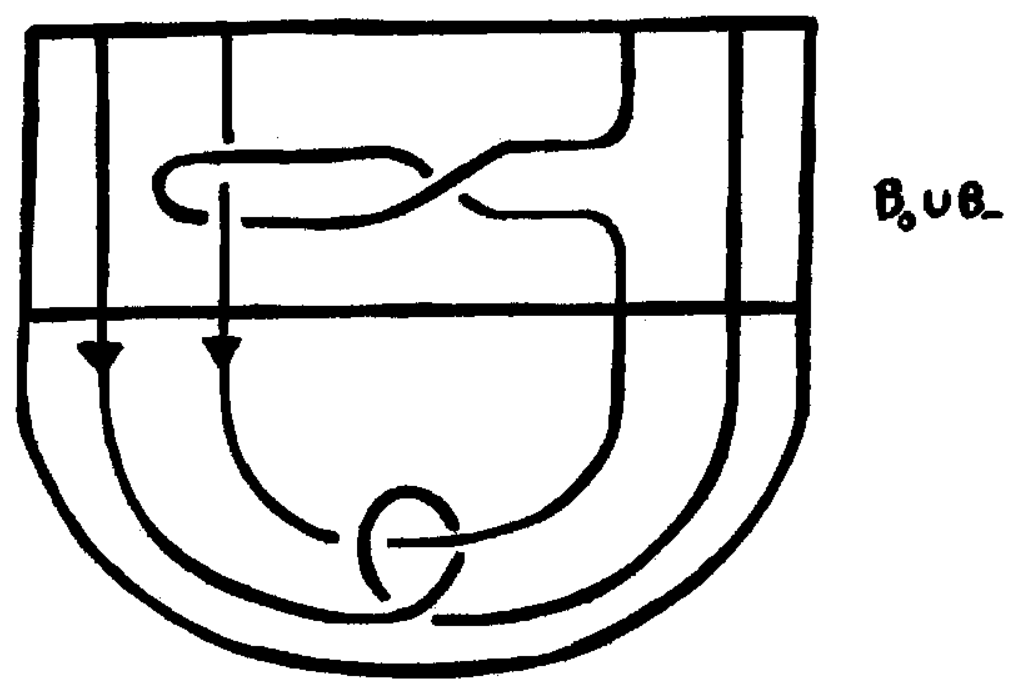

Figura 34

Empurrando $B_{0}$ em uma vizinhança colar de $B$, podemos identificar $B_{0} \cup B_{-}$com $B_{-}$. O mergulho resultante de $\underline{k} \times I \mathrm{~cm} B$ é um $k$-enlaçamento de intervalos, que será denotado por $\beta \cdot f$. Como parte da i-ésima corda de $\beta \cdot f$, a orientação de $\beta(i \mathrm{x} I)$ para $i \in\{k+1, \ldots, 2 k\}$ foi invertida.

Denotemos por $j_{i}^{\prime}$ o $k$-enlaçamento de intervalos trivial visto como um mergulho $j_{0}^{\prime}: \underline{k} \times I \rightarrow B_{+}$tal que $j_{0}^{\prime}(i, 0)-\tilde{p}_{i} \in D \times\{1 / 4\}$ e $j_{0}^{\prime}(i, 1)=p_{i} \in D \times\{1\}$.

A "união" de $j_{0}^{\prime} \operatorname{com} \beta \cdot f$, visto como o mergulho original de $\underline{k} \times I$ em $B_{0} \cup B$, no caso particular em que $\beta$ é o $2 k$-enlaçamento de intervalos trivial, é um enlaçamento de $k$ componentes que ć chamado o fecho do $k$-enlaçamento de intervalos $f$ e será denotado por $\hat{f}$. Veja figura 35 . 


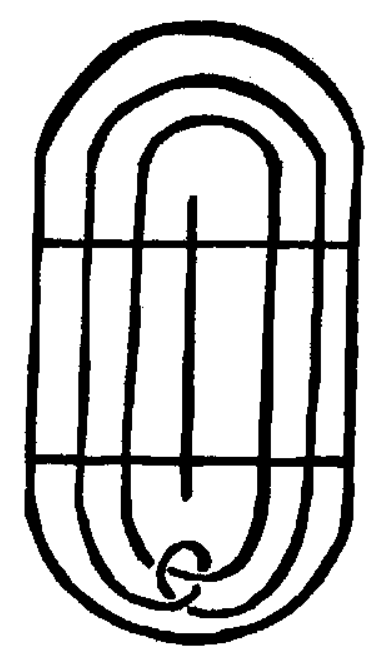

Figura 35

Se em $D \times I \times I$ identificarmos, $\forall z \in D, \forall s \in I,(z, 0, s) \operatorname{com}(z, 1, s)$ e, $\forall t \in I$, $\forall s \in I$, identificarmos $(z, t, s) \operatorname{com}(z, 1 / 4-t(\bmod 1), s)$ para todo $z-(x, y) \in \partial D$ com $x \geq 0$ e $y \leq 0$, obtemos um espaço identificação que é homeomorfo a $B \times I$.

Portanto, se $f$ e $g$ são $k$-enlaçanentos de intervalos considerados como os mergulhos $f, g: \underline{k} \times I \rightarrow B_{\ldots}$, um cobordismo entre $f$ e $g$ pode ser considerado como um mergulbo

$$
F: \underline{k} \times I \times I \rightarrow B \times I \text { tal que }\left.F\right|_{\underline{k} \times I \times\{0\}}=f,\left.F\right|_{\underline{k} \times I \times\{1\}}=g
$$

e $\forall i \in \underline{k}, \forall s \in I, F^{\prime}(i, 0, s)=\left(p_{i}^{\prime}, s\right)$ e $F(i, 1, s)=\left(\ddot{p}_{i}^{\prime}, s\right)$

Analogamente, se $\beta$ e $\gamma$ são $2 k$-enlaçamentos de intervalos considerados como mergulhos $\beta, \gamma: 2 \underline{k} \times I \rightarrow B_{0}$, então um cobordismo entre $\beta$ e $\gamma$ pode ser considerado como um mergulho

$$
\Sigma: 2 \underline{k} \times I \times I \rightarrow B_{0} \times I \text { tal que }\left.\Sigma\right|_{2 \underline{k} \times I \times\{0\}}=\beta,\left.\Sigma\right|_{2 \underline{k} \times I \times\{1\}}=\gamma
$$

$\mathrm{c}, \forall i \in \underline{k}, \forall s \in I, \Sigma(i, 0, s)-\left(p_{i}, s\right), \Sigma(1, i, s)=\left(p_{i}^{\prime}, s\right), \nu(k+i, 0, s)=\left(\tilde{p}_{k-i+1}, s\right) \mathrm{e}$ $\Sigma(k+i, 1, s)-\left(\tilde{p}_{k-\imath \uparrow 1}^{\prime}, s\right)$.

A "união" de $F$ e $\Sigma$ nos dá um mergulho de $\underline{k} \times I$ x $I$ em $\left(B_{0} \cup B_{-}\right)$x $I$, que também pode ser considerado como um mergulho $\Sigma \cdot F: \underline{k} \times I \times I \rightarrow B_{-} \times I$, se nós identificarmos 
$B_{0} \times I$ L $B_{-} \times I$ com $B_{-} \times I$ empurrando $B_{0} \times I$ em uma vizinhança colar de $B_{-} \times I$. $\Sigma \cdot F$ é claramente um cobordismo entre $\beta \cdot f \mathrm{e} \gamma \cdot g$.

Isto define uma ação do grupo $C_{1,2 k}$ no conjunto $C_{1, k}$ que será chamada Ação de Habbeger-Lin.

No caso particular em que $\Sigma$ é o cobordismo trivial $j_{0} \times i d_{I}: 2 \underline{k} \times I \times I \rightarrow B_{0} \times I$ entro o $2 k$-enlaçamcrito trivial $j_{0}$ e ele mesmo, a "união" de $\Sigma \cdot F$, como o mergulho original de $\underline{k} \times I \times I$ em $\left(B_{0} \cup B_{-}\right) \times I$, com $j_{0}^{\prime} \times i d_{l}$, onde $j_{0}^{\prime}: k \times I \rightarrow B_{+}$é o k-enlaçamento de intervalos trivial, fonece um cobordismo $\hat{F}: \underline{k} \times S^{1} \times I \rightarrow B \times I \subset S^{3} \times I$ entre $\hat{f}$ e $\hat{g}$, o qual será chamado de fecho do cobordismo $\mathrm{F}$ de $k$-enlaçamentos de intervalos.

Definição 4.2.1. Seja $D$ com sua orientaçäo usual e seja $L \subseteq S^{3}$ um enlaçamento orientado e ordenado com $k$ componentes. Se $j: D \rightarrow S^{3}$ é um mergulho tal que, para Lodo $i \in \underline{k}$, a interseção de $j(D)$ com a $i$-ésima componente de $L$ é $j\left(a_{2}\right)$ e todos os número de interseções são 1 , então dizemos que $j(D)$ é uma d-base para $L$ e que $(L, j(D))$ é um enlaçamento d-baseado.

Dado um k-enlaçamento de intervalos $f$ devemos considerar que seu fecho possui uma d-base padrão $j_{0}(D)-D_{1-\varsigma} \times\{1 / 8\}$, onde $\epsilon>0$ é pequeno suficiente tal que, $\vee i \in \underline{k}$, $p_{i} \in D_{1}$, $x\{0\}$, onde $D_{1-\text { e é }}$ 2-disco com raio $1-c$ e $j_{0}: D \rightarrow D_{1-\epsilon} \times\{1 / 8\} \subseteq B \subseteq S^{3}$ co o mergulho definido por $j_{0}(z)=((1-\epsilon) z, 1 / 8)$.

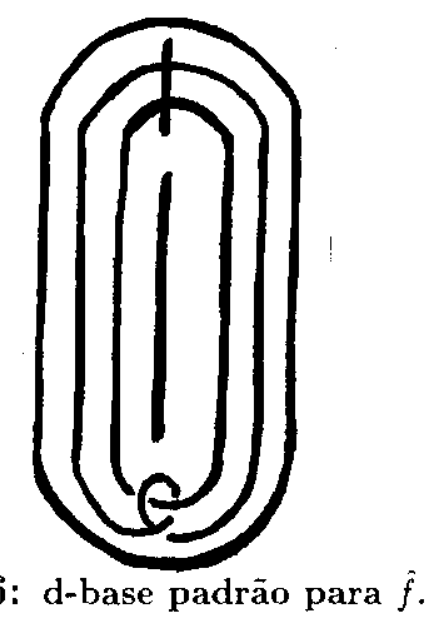

Figura 36: d-base padrāo para $\hat{f}$.

Assim obtemos uma aplicação $p$ das classes do isotopia de enlaçamentos de intervalos para as classes de isotopia de enlaçamentos d-baseados, a qual é uma bijeção [8]. $\varphi$ será chamada a bijeção de Habegger-Lin. 


\section{Capítulo 5}

\section{Enlaçamentos de Intervalos Bordantes}

\subsection{Enlaçamentos de Intervalos Bordantes.}

Definição 5.1.1. Sejam $f$ um k-enlaçamento de inlervalos e $X(f)$ seu exterior. O grupo $\Pi_{1}(X(f), p)$, onde $p=(0,-1,0)$ é chamado o grupo de $f$ e será denotado por $\Pi_{1}(f)$.

Denotaremos por $x_{i}=x_{i}(f), \forall i \in \underline{k}$, o clemento de $\Pi_{1}(f)$ representado pela composição dos caminhos $t_{i} m_{i} t_{i}^{-1} \in X(f)$, onde $m_{i}$ é um círculo no sentido anti-horário de raio $r<\frac{1}{k+1}$ ao redor do ponto $\left(a_{i}, 0\right)$ e ti é um segmento de reta de $p=(0,-1,0)$ ao ponto $\left(-1+\frac{2 i}{k+1},-r, 0\right)$. (Veja figura 37$)$.

Denotaremos por $y_{i}=y_{i}(f), \forall i \in \underline{k}$, o elemento de $\Pi_{1}(f)$ representado pela composição dos caminhos $u t_{i}^{\prime} m_{i}^{\prime} t_{i}^{\prime-1} u^{1}$, ondo $m_{i}^{\prime}$ é um ć́rculo no sentido anti-horário de raio $r<\frac{1}{k+1}$ ao redor do ponto $\left(a_{i}, 1\right)$ e $t_{i}^{\prime}$ é um segmento de reta de $(0,-1,1)$ ao ponto $\left(-1+\frac{2 i}{k+1},-r, 1\right)$ e ú ú segmento de retá de $p$ à $(0,-1,1)$. (Veja figura 37$)$.

Definição 5.1.2. Seja $H^{\prime}(k)-F\left(\alpha_{1}, \alpha_{2}, \ldots, \alpha_{k}\right)$ o grupo livre com $k$ geradores $\alpha_{1}, \alpha_{2}, \ldots, \alpha_{k}$ e seja $f$ um $k$-enlaçamento de intervalos. O homomorfismo $\mu_{0}(f): F(k) \rightarrow \Pi_{1}(f)$ definido por $\mu_{0}(f)\left(\alpha_{i}\right)=x_{i}, \forall i \in \underline{k}$, é chamado aplicação meridiano canônica para $f$ no nível 0 ou aplicação meridiano do topo para $f$ e o homomorfismo $\mu_{1}(f): F(k) \rightarrow \Pi_{1}(f)$ definido por $\mu_{1}(f)\left(\alpha_{i}\right)=y_{i}, \forall i \in \underline{k}$, é chamado aplicação meridiano canônica para $f$ no nível 1 ou aplicação meridiano da base para $f$. OS $x_{i}, i \in \underline{k}$, serão chamados de meridianos do topo e os $y_{i}, i \in \underline{k}$, de meridianos da base para $f$. 


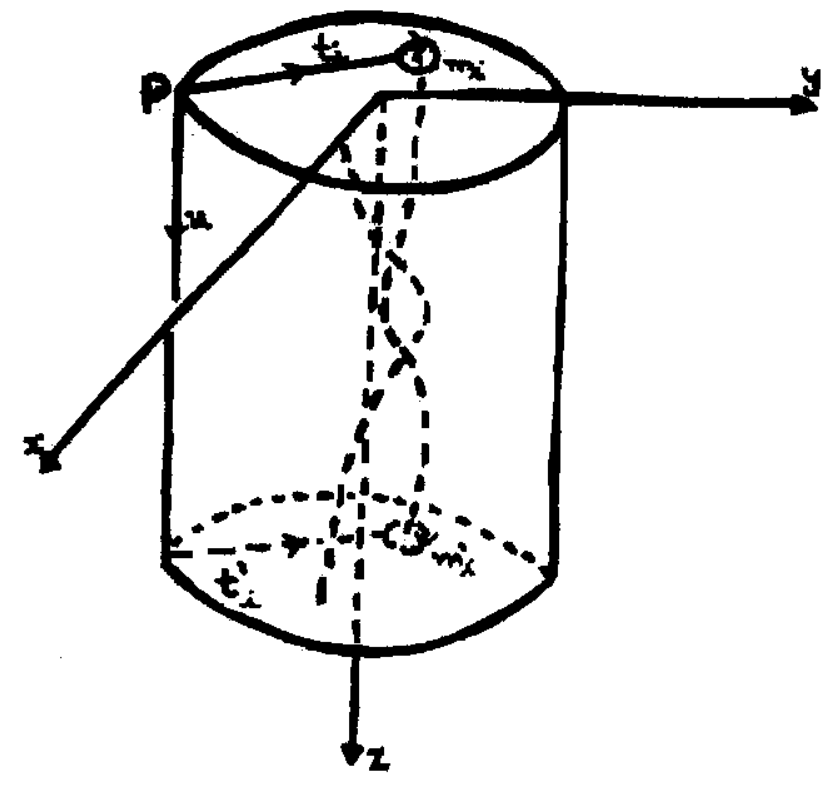

Figura 37

Definição 5.1.3. Dado um k-enlaçamento de intervolos $f$; um epimorfismo $\eta(f): \Pi_{1}(f) \rightarrow$ $F^{\prime}(k)$ tal que $\eta(f) \mu_{0}(f)=i d_{F(k)}=\eta(f) \mu_{1}(f)$ é chamado uma aplicação splitting (ou cisão) para $f$. Un enlaçamento de intervalos que possui uma aplicą̧ão splitting é dito ser urn enlaçamento de intervalos bordante.

Pela Proposição 1 de [5], pág 860, o splitting $\eta(f)$ do enlaçamento de intervalos bordantes $f$ éninico.

Para todo $i \in \underline{k}$, seja $A_{i}=\partial(D \times I) \cap S$, onde $D$ é o disco unitário em $\mathbb{R}^{2}$ e $S \subseteq \mathbb{R}^{3}$ é o semiplano consistindo dos pontos $(x, y, z)$ com $y \geq 0$ contido no plano passando por $\left(a_{i}, 0\right)$ e perpendicular ao eixo $0 x$. (Veja figura 38 ).

No próximo resultado denotaremos um $k$-enlaçamento de intervalos $f: \underline{k} \times I \rightarrow D \times I$ por $f_{1} \cup f_{2} \cup \cdots \cup f_{k}$, onde para todo $i \in \underline{k}, f_{i}=\left.f\right|_{\{i\} \times \text { I } I}$ ć a $i$-ésima corda de $f$.

Proposição 5.1.4. Um k-enlaçamento de intervalos $f=f_{1} \cup f_{2} \cup \cdots \cup f_{k}$ é um enlaçamento de intervalos bordante se, e somente se, para todo $i \in \underline{k}$, existir uma superfície orientada e conexa com bordo $S_{\imath} \subseteq D \times I$ de modo que $\partial\left(S_{i}\right)=i m f_{i} \cup A_{\imath}, S_{i} \cap S_{j}=\emptyset$ se $i \neq j$ e $S_{i} \backslash i m f_{i}$ é uma subvartedade própría de $D \times I$. 


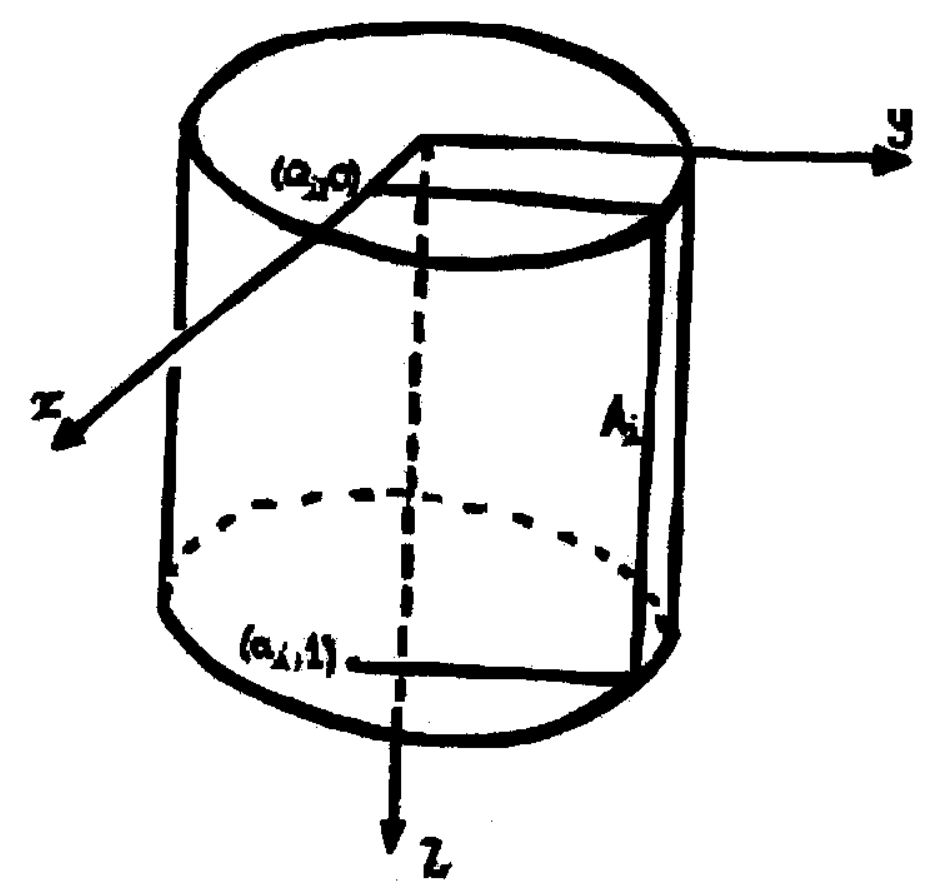

Figura 38

\section{Demonstraçāo:}

Suponhamos que existam as superfícies $S_{i}$ como acima. Sejam $S_{f}=\bigcup_{i=1}^{k} S_{i}$ e $\bar{S}_{f}=$ $S_{f} \cap X(f)$. A construção de Thom-Pontryagin para o par $\left(\bar{S}_{f}, \partial \bar{S}_{f}\right)$ em $(X(f), \partial X(f))$ fornece uma aplicação $P(f): X(f) \rightarrow \bigvee_{i=1}^{k} S^{1}$ tal que $P(f)_{*} \mu_{0}(f)=i d_{F(k)}=P(f)_{*} \mu_{1}(f)$. Portanto, $f$ ć um $k$-enlaçamento do intervalos bordarite com splitting $P(f)_{*}$.

Reciprocamente, suponhamos que $f$ é um $k$-enlaçamento de intervalos bordante. Seja $X\left(j_{0}\right)=D_{k} \times I$, onde $D_{k}$ é um disco com $k$ pequenos discos abertos removidos. Projetando $D_{k} \times I$ em $D_{k} \times\{0\}$ e retraindo $D_{k} \times\{0\}$ em $\bigvee_{i=1}^{k} S^{1}$, obtemos uma aplicação $P: \partial X\left(j_{0}\right) \rightarrow \bigvee_{i=1}^{k} S^{1}$

Por outro lado, $f$ induz um mergulho $h: \partial X\left(j_{0}\right) \stackrel{\cong}{\longrightarrow} \partial X(f) \subset X(f)$.

Como $\mathrm{II}_{1}\left(\partial X\left(j_{0}\right), p\right)$ é gerado por $\left\{x_{i}, y_{i} / i \in k\right\}$ e $P_{*}: \mathrm{II}_{1}\left(\partial X\left(j_{0}\right)\right) \rightarrow F(k)$ leva $x_{i} \mathrm{e}$ $y_{i}$ no mesmo gerador $\alpha_{i}$ de $F(k)$, o seguinte diagrama é comutativo: 


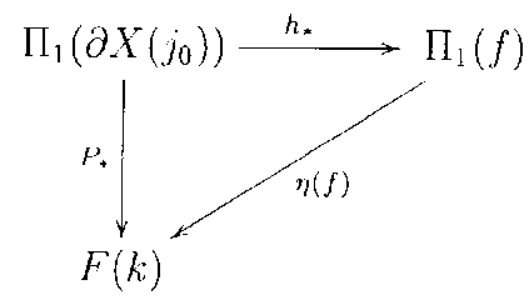

Como $\bigvee_{i=1}^{k} S^{1}$ ć um $K(F(k), 1)$, existe uma aplicação $P(f): X(f) \rightarrow \bigvee_{i=1}^{k} S^{1}$ tal que $P(f)_{*}=\eta(f)$. Usando o teorema de extensão de homotopia, podemos supor que $P(f)$ comuta o diagrama:

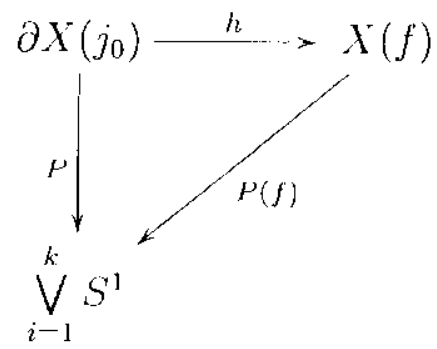

e estendendo os espaços neste diagrama, podemos supor que $P(f)$ é uma aplicação diferenciável.

A transversalidade de $P(f)$ em $k$ pontos distintos, um de cada círculo de $\bigvee_{i=1}^{k} S^{1}$, nos fornece $k$ superfícies $\tilde{S}_{1}, \ldots, \tilde{S}_{k}$ disjuntas duas a duas.

Desprezando as componentes fechadas de $\tilde{S}_{f}=\bigcup_{i-1}^{k} \hat{S}_{i}$ se necessário, obtemos a superfície desejada $S_{f}=\bigcup_{i=1}^{k} S_{i}$ adicionando colares a $\tilde{S}_{f}$.

Definiçāo 5.1.5. Uma superficie $S_{f}=\bigcup_{i=1}^{k} S_{i}$ nas condições da proposição anterior é chamada uma BL-superfície de Seifert para o $k$-enlaçamento de intervalos $f$.

Definiçāo 5.1.6. Sejam $f_{1}$ e $f_{2}$ k-enlaçamentos de intervalos e $F$ um cobordismo entre eles. $F$ é chamado um cobordismo bordante se existe um epimorfismo $\eta(F): \mathrm{II}_{1}(F)=$ $\Pi_{1}(X(F)) \rightarrow F(k)$, onde $X(F)$ é o exterior de $F$, tal que, $\forall i \in\{1,2\}, \eta\left(f_{i}\right)=\eta(F) j_{i}$, onde $j_{i}: \Pi_{1}\left(f_{i}\right) \rightarrow \Pi_{1}(F)$ säo aplicações induzidas das inclusões. $\eta(F)$ é dito ser um splitting (ou cisão) para o cobordismo $\mathrm{F}$.

Proposição 5.1.7. O conjunto $B S L(k)$ das classes de cobordismo bordante de $k$-enlaçamentos de intervalos bordantes, com o produto usual de enlaçamentos de intervalos, é um grupo. 


\section{Demonstraçāo:}

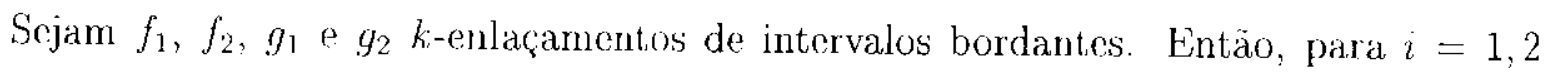
existem

$$
\eta\left(f_{i}\right): \Pi_{1}\left(f_{i}\right) \rightarrow F(k) \text { e } \eta\left(g_{i}\right): \Pi_{1}\left(g_{i}\right) \rightarrow F(k)
$$

tal que $\eta\left(f_{i}\right) \mu_{0}\left(f_{i}\right)=i d_{F(k)}=\eta\left(f_{i}\right) \mu_{i}\left(f_{i}\right)$ : $\eta\left(g_{i}\right) \mu_{0}\left(g_{i}\right)-i d_{F(k)}=\eta\left(g_{i}\right) \mu_{1}\left(g_{i}\right)$. Pelo teorema de Seifert-Van Kampen, $\forall i \in\{1,2\}$, obtemos o seguinte diagrama push-out,

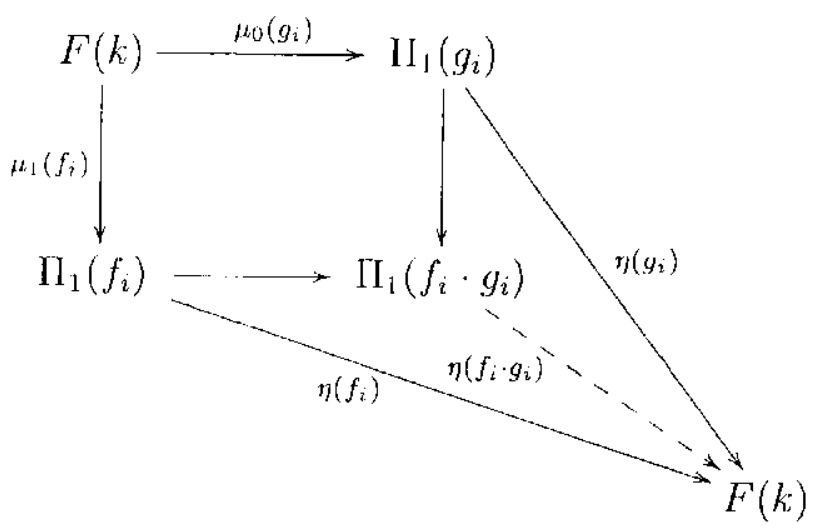

Então a aplicação $\eta\left(f_{i} \cdot g_{i}\right)$ é um splitting para $\int_{i} \cdot g_{i}, \log f_{i} \cdot g_{i}$ também é um $k$-enlaçamento de intervalos bordantes.

Se $F: \underline{k} \times I \times I \rightarrow D \times I \times I$ é um cobordismo entre $f_{1}$ e $f_{2}$ e $G$ é um cobordismo entre $g_{1}$ e $g_{2}$, então podemos definir um cobordismo entre $f_{1} \cdot g_{1}$ e $f_{2} \cdot g_{2}$. Para isso, consideremos as aplicaçóes $\alpha_{1}, \alpha_{2}: D \times I \rightarrow D \times I$ dadas por $\alpha_{1}(x, t)=(x, t / 2) \mathrm{e}$ $\alpha_{2}(x, t)=(x,(l+1) / 2)$.

Definimos $F \cdot G: \underline{k} \times I \times I \rightarrow D \times I \times I$ por

$$
F \cdot G(i, t, s)= \begin{cases}\left(\alpha_{1} \times i d_{I}\right) F(i, 2 t, s) & \text { se } 0 \leq t \leq 1 / 2 \\ \left(\alpha_{2} \times i d_{I}\right) G(i, 2 t-1, s) & \text { se } 1 / 2 \leq t \leq 1\end{cases}
$$

Entāo $F \cdot G$ é um cobordismo entre $f_{1} \cdot g_{1} e f_{2} \cdot g_{2}$. Além disso, se $F$ e $G$ são cobordismos bordantes temos o seguinte diagrama push-out 
(2)

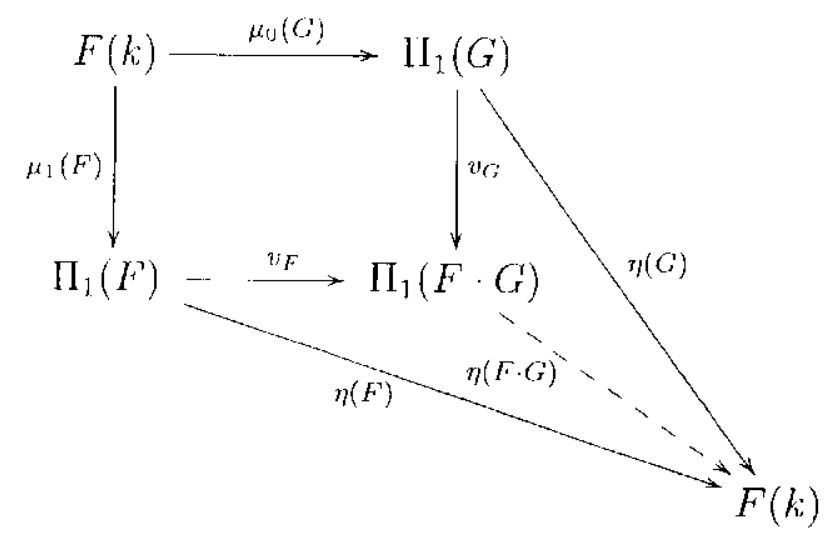

onde, para todo $i \in \underline{k}, v_{F}\left(x_{i}(F)\right)=x_{i}\left(F^{\prime} \cdot G\right)$ e $v_{G}\left(y_{i}(G)\right)-y_{i}(F \cdot G)$.

Portanto $\eta(F \cdot G)\left(x_{i}(F \cdot G)\right)=\eta(F \cdot G) v_{F}\left(x_{i}(F)\right)=\eta(F)\left(x_{i}(F)\right)=\alpha_{i}$ c analogamente, $\eta(F \cdot G)\left(y_{i}(F \cdot G)\right)=\alpha_{i}$. Além disso, o diagrama abaixo é comutativo, para todo $i \in\{1,2\}$,

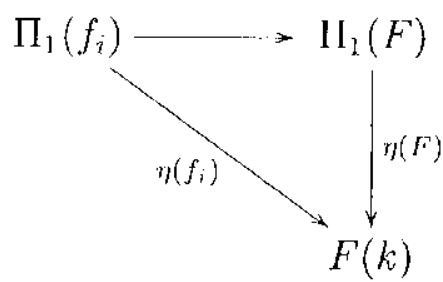

que junto com o diagrama (2) nos fornece o seguinte diagrama comutativo $(i \in\{1,2\})$ :

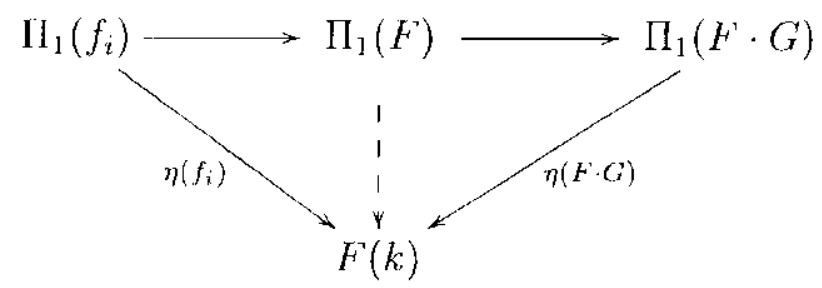

que ao junto com o diagrama (1) fornece o diagrama comutativo $(i \in\{1,2\})$ :

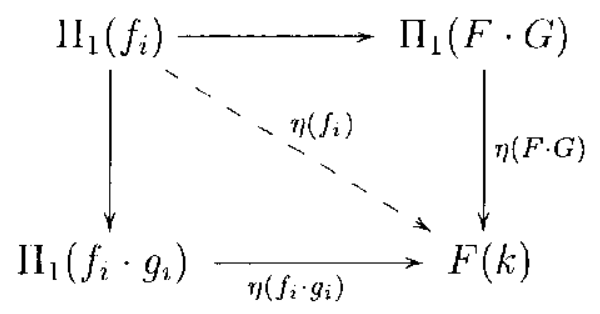

Finalmente, o diagrama (3) junto com o diagrama comutativo abaixo $(i \in\{1,2\})$ : 


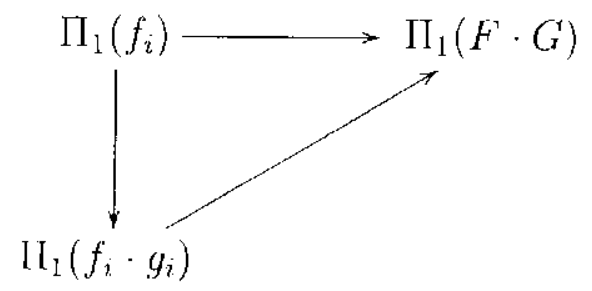

fornece o diagrama comutativo abaixo $(i \in\{1,2\})$ :

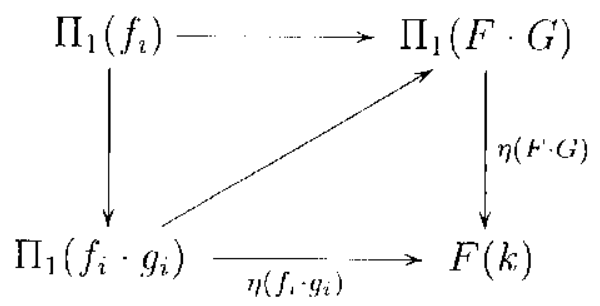

ou seja, para todo $i \in\{1,2\}$ obtemos diagramas comutativos:

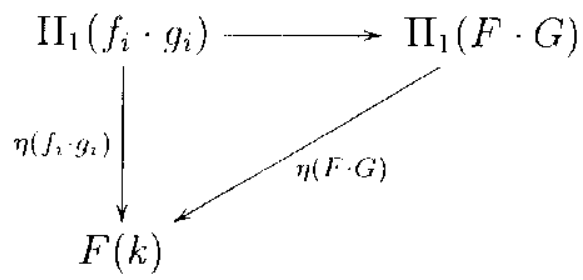

Portanto, $F \cdot G$ é um cobordismo bordante cntre os enlaçamentos de intervalos bordantes $f_{1} \cdot g_{1}$ e $f_{2} \cdot g_{2}$.

$O$ inverso $f^{-1}$ de um enlaçamento de intervalos bordante $f$ tiambém é um enlaçamento de intervalos bordante. Além disso, existe um cobordismo $F$, dado na Proposição 4.1.4, entre $f \cdot f^{-1}$ (ou $f^{-1} \cdot f$ ) e $j_{0}$, cuja imagem tem complemento homeomorfo ao complemento da imagem de $f \times d_{1}$. Portanto, $F$ é um cobordismo bordante entre $f \cdot f^{-1}$ e o enlaçamento de intervalos trivial $j_{0}$.

\subsection{Enlaçamentos de Intervalos Bordantes e Enlaçamentos $F(k)$.}

Seja $f$ um k-enlaçamento de intervalos e seja $\hat{f}$ seu fecho. $\Pi_{1}(\hat{f})$ é o quociente de $\Pi_{1}(f)$ pelo subgrupo normal gerado por $\left\{y_{i} x_{i}^{-1} / i \in \underline{k}\right\} . \mu(f)$ denotará $\mu_{0}(f)$ ou $\mu_{1}(f)$. Se $q: \Pi_{1}(f) \rightarrow \Pi_{1}(\hat{f})$ é o epimorfismo canônico, então $\widehat{\mu(f)}=q \mu(f): F(k) \rightarrow \Pi_{1}(\hat{f})$ é uma aplicação meridiano para o enlaçamento $\hat{f}$. Além disso, o diagrama 


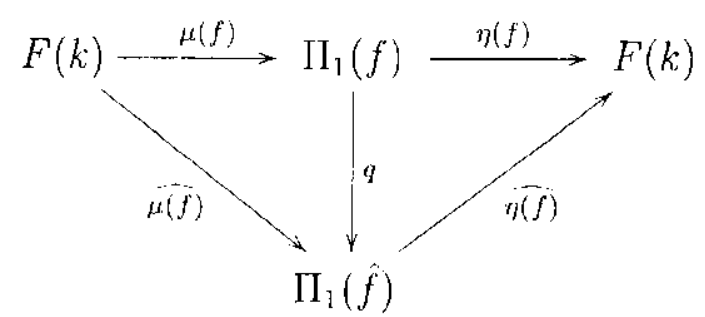

mostra que $\widehat{\mu(f)}$ tem um splitting $\widehat{\eta(f)}$ se, e somente se, $\mu(f)$ tem um splitting $\eta(f)$.

Proposição 5.2.1. Existe uma aplicação sobrejetora $\psi$ de $B S L(k)$ no conjunto $C_{1}(F(k))$ de classes de $F(k)$-cobordismos de enlaçamentos $F(k)$.

\section{Demonstração:}

Se $f$ é um $k$-enlaçamento de intervalos bordante, pela nota prévia, $\widehat{\mu(f)}$ possui um splitting $\widehat{\eta(f)}$. Portanto, existe uma aplicação $f \mapsto(\hat{f}, \widehat{\eta(f)})$ de $k$-enlaçamentos de intervalos bordante (m) $F(k)$-enlaçamentos.

Dado um $F(k)$-enlaçamento $(L, \eta)$, seja $\eta: F(k) \rightarrow \mathrm{I}_{1}(L)$ uma aplicação meridiano com splitting $\eta$. Seja $D_{k}$ um disco com $k$ pequenos abertos removidos, cada um centrado erm um dos pontos $a_{i} \in D$. Seja $X(L)$ o exterior de $L$. Então $\mu$ pode ser realizada por um mergulho $i: D_{k} \rightarrow X(L)$, o qual pode ser estendido ao mergulho $j: D \rightarrow S^{3}$ tal que $j(D)$ é uma d-base para $L$.

Seja $f$ o enlaçamento de intervalos que corresponde ao cnlaçamento d-baseado $(L, j(D))$ pela bijeção de Habegger-Lin. Em particular, $\hat{f}=L$. Nossa cscolha da d-base ć tal que $\eta=\widehat{\eta(f)}$, isto é, $\eta$ é um splitting para $\widehat{\mu(f)}$. Portanto, $f$ é um enlaçamento de intervalos bordante e nossa aplicação $f \mapsto(\hat{f}, \widehat{\eta(f)})=(L, \eta)$ é sobrejetora.

Sejam $f, g$ enlaçamentos do intervalos bordante e $F$ um cobordismo bordante entre eles com splitting $\eta(F)$. Como vimos na seçāo 4.2 , fechando $F$ obtemos o cobordismo $\hat{F}$ entre $\hat{f}$ e $\hat{y}$. Além disso, como o diagrama

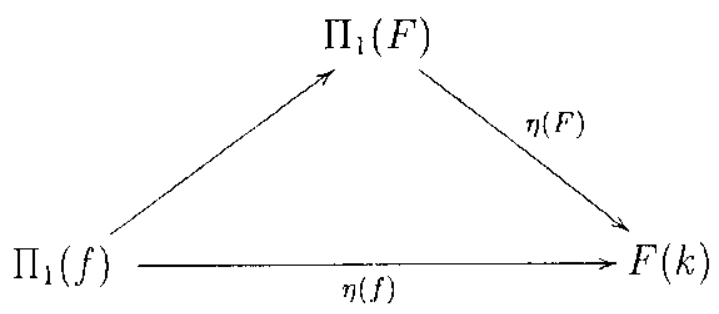

é comutativo e, $\forall i \in \underline{k}, \eta(f)\left(x_{i}\right)=\eta(f)\left(y_{i}\right)$, segue que $\forall i \in \underline{k}, \eta(F)\left(\left(x_{i}(F)\right)=\right.$ $\eta(F)\left(\left(y_{i}(F)\right)\right.$. 
Como $i m(\hat{F})$ ć obtida de $i m(F)$ identificando $D \times 0 \times I$ com $D \times 1 \times I$ (o resultado sendo mergulhado em $\left.S^{3} \times I\right)$, segue que $\eta(F)$ induz $\widehat{\eta(F)}: \Pi_{1}(\hat{F}) \rightarrow F(k)$, o qual estende $\widehat{\eta(f)}$ e $\widehat{\eta(g)}$. Portanto $(\hat{f}, \widehat{\eta(f)})$ e $(\hat{g}, \widehat{\eta(g)})$ são $F(k)$-cobordantes. Então obtemos umà aplicação $\psi: B S L(k) \rightarrow C_{1}(F(k))$ e $\psi$ é sobrejetora.

\subsection{Enlaçamentos de Intervalos Bordantes e Tranças Puras.}

Usando o procedimento de Habbeger-Lin, descrito no Capítulo 4, mas deixando $\beta$ variar somente entre as tranças puras, temos uma açäo do grupo $P_{2 k}$ das tranças puras com $2 k$ cordas no conjunto $S L(k)$ de (classes de isotopia de) $k$-enlaçamentos de intervalos. Seja $\left(P_{2 k}\right)_{j_{0}}$ o estabilizador do enlaçamento de intervalos trivial jo para esta ação, isto é, $\left(P_{2 k}\right)_{j_{0}}=\left\{\beta \in P_{2 k} \mid \beta \cdot j_{0}=j_{0} \subset S L(k)\right\}$.

Seja $C A_{k}$ o grupo de todos os automorfismos de um grupo livre $F(k)$ com $k$ geradores $\alpha_{1}, \ldots, \alpha_{k}$ que leva cada $\alpha_{i}$ em um de seus conjugados.

Proposição 5.3.1. Fxiste um epimorfismo $\theta:\left(P_{2 k}\right)_{j 0} \rightarrow C^{r} A_{k}$.

\section{Demonstraçāo:}

Seja $f$ uma trança pura com $k$ cordas e realizemos as aplicações meridianos $\mu_{0}$ do topo e $\mu_{1}$ da base, através do mergulhos $\lambda_{0}, \lambda_{1}: D_{k} \rightarrow X(f)$, respectivamente, onde $D_{k}$ ć um disco com $k$ furos. Então $\lambda_{1}^{-1}: i m \lambda_{1} \rightarrow D_{k}$ estende-se a uma aplicação diferenciável $\lambda(f): X(f) \rightarrow D_{k}$ e, pelo Teorema 1.2.1 (Teorema da Representaçāo de Artin), $\lambda(f) \lambda_{0}$ induz um automorfismo $\bar{f}=\left(\lambda(f) \lambda_{0}\right)_{*}=\lambda(f)_{*} \mu_{0}(f) \in H_{0}(F(k))$, onde $H_{0}(F(k))$ é o subgrupo de $C A_{k}$ consistindo daqueles autornorfismos que fixam o produto $\alpha_{1}, \alpha_{2}, \ldots, \alpha_{k}$. Além disso, vimos também no Teorema 1.2.1 que a aplicação de $P_{k}$ em $H_{0}(F(k))$ que leva $f$ em $\bar{f}$ é um isomorfismo de grupos.

Seja $\beta \in\left(P_{2 k}\right)_{j u}$ e denotemos por $x_{1}, \ldots, x_{k}, \tilde{x}_{k}, \ldots, \tilde{x}_{1}$ os meridianos do topo e por $y_{1}, \ldots, y_{k}, \tilde{y}_{k}, \ldots, \tilde{y}_{1}$ os meridianos da base de $\beta$. Denotemos ainda por $\alpha_{1}, \ldots, \alpha_{k}, \tilde{\alpha}_{k}, \ldots, \tilde{\alpha}_{1}$ os geradores de $H^{\prime}(2 k)=\mathrm{I}_{1}\left(D_{2 k}\right)$. 


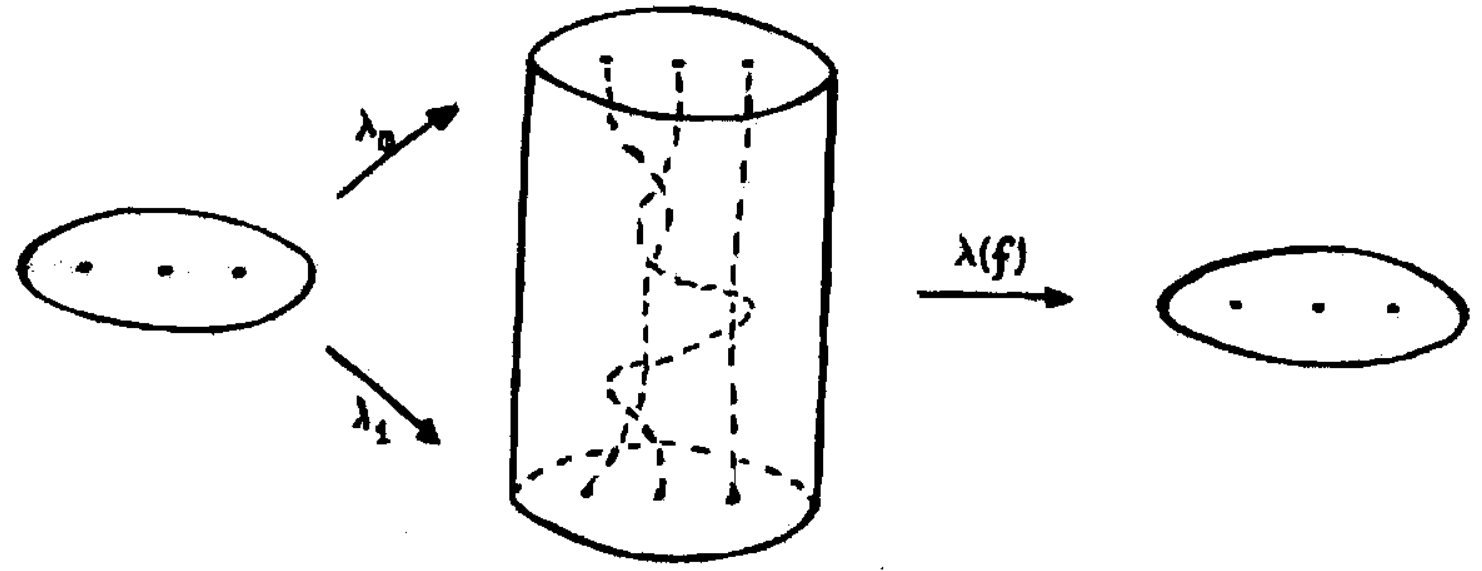

Figura 39

Desde que o isomorfismo $\lambda(\beta)_{*}: \Pi_{1}(\beta) \rightarrow F(2 k)$ leva $y_{i}$ em $\alpha_{i}$ e $\tilde{y}_{i}$ em $\tilde{\alpha}_{i}$, ele induz um isomorfismo

$$
\lambda(\beta)_{*}: \frac{\prod_{1}(\beta)}{\left\langle y_{i} \tilde{y}_{2} / i \in k\right\rangle^{N}} \longrightarrow \frac{F(2 k)}{\left\langle\alpha_{i} \bar{\alpha}_{i} / i \in \underline{k}\right\rangle^{N}}
$$

onde $\left\langle y_{i} \tilde{y}_{i} / i \in \underline{k}\right\rangle^{N}$ é o subgrupo normal de $I_{1}(\beta)$ gerado por $\left\{y_{i} \tilde{y}_{i} / i \in \underline{k}\right\}$ e $<\alpha_{i} \bar{\alpha}_{i} / i \in \underline{k}>^{N}$ é o subgrupo normal de $F(2 k)$ gerado por $\left\{\alpha_{i} \tilde{\alpha}_{i} / i \in \underline{k}\right\}$.

Pelo teorema de Seifert-Van Kampen, temos o seguinte diagrama push-out

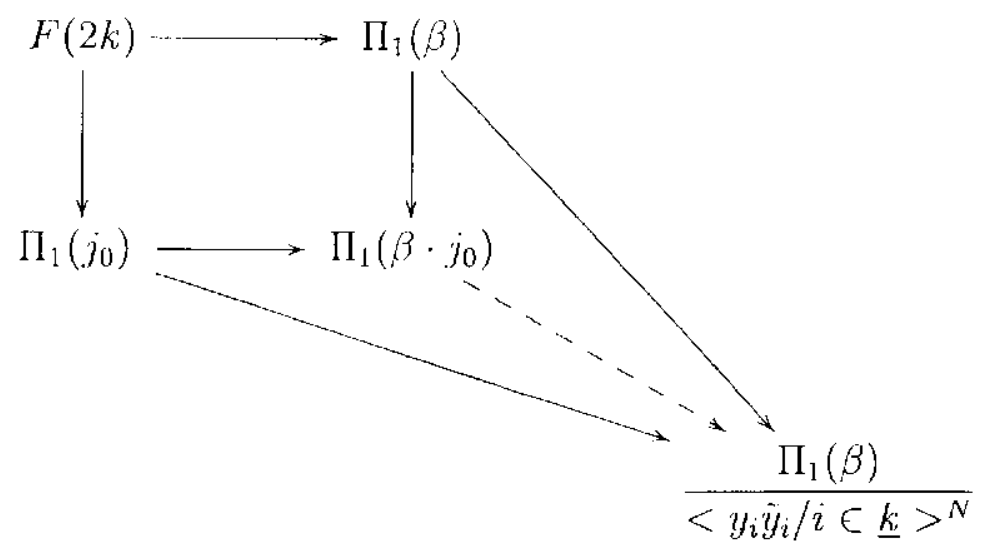

Segue que $\Pi_{1}\left(\beta \cdot j_{0}\right)$ é isomorfo a $\frac{I_{1}(\beta)}{\left\langle y_{i} \bar{y}_{i} / i \in \underline{k}\right\rangle^{N}}$ tendo meridianos $\left[x_{i}\right]$ no topo e $\left[\tilde{x}_{i}^{-1}\right]$ na base: onde [ ] denota a classe de equivalência no grupo quociente.

Pcla definiçào de $\lambda\left(\beta \cdot j_{0}\right): X\left(\beta \cdot j_{0}\right) \rightarrow D_{k}, \lambda\left(\beta \cdot j_{0}\right)_{*}\left(\left[\tilde{x}_{i}^{-1}\right]\right)=\alpha_{i}$ e como $\beta \in\left(P_{2 k}\right)_{j_{0}}$ 
$\lambda\left(\beta \cdot j_{0}\right)_{*}\left(\left[x_{i}\right]\right)=\alpha_{i}$. Como $\lambda\left(\beta \cdot j_{0}\right)_{*}$ é um isomorfismo, $\left[\tilde{x}_{i}\right]^{{ }^{\prime 1}}=\left[x_{i}\right]$.

$\Lambda$ aplicação meridiano do topo de $\beta, \mu_{0}(\beta): F(2 k) \rightarrow \Pi_{1}(\beta)$ leva $\alpha_{i} \tilde{\alpha}_{i}$ em $x_{i} \tilde{x}_{i}$ e desde que $\left[x_{i} \bar{x}_{i}\right]=1 \mathrm{em} \frac{\Pi_{l}(\beta)}{\left\langle y_{i} \bar{y}_{i} / i \in \underline{k}\right\rangle^{N}}, \mu_{0}(\beta)$ induz um homomorfismo

$$
\mu_{0}(\beta): \frac{F^{\prime}(2 k)}{\left\langle\alpha_{i} \tilde{\alpha}_{i} / i \in \underline{k}\right\rangle^{N}} \rightarrow \frac{\Pi_{1}(\beta)}{\left\langle y_{i} \tilde{y}_{i} / i \in \underline{k}\right\rangle^{N}}
$$

Portanto, temos o seguinte diagrama comutativo

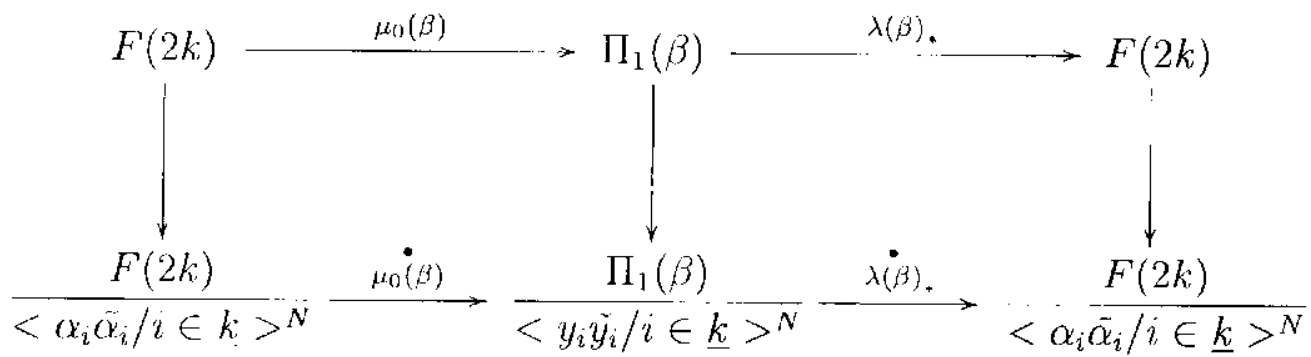

onde as aplicaçōes verticais são homomorfismos quociente.

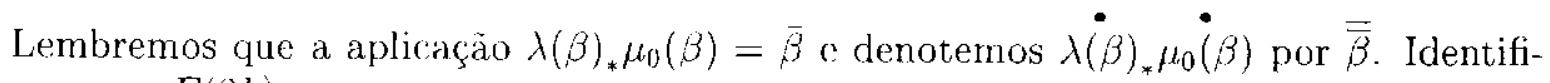
cando $\frac{F(2 h)}{\left\langle\alpha_{i} \alpha_{i} / i \in \underline{k}\right\rangle^{N}}$ com $F(k)$ através do isomorfismo

$$
F(k) \rightarrow \frac{F(2 k)}{\left\langle\alpha_{i} \tilde{\alpha}_{i} / i \in k\right\rangle^{N}}
$$

que leva $\alpha_{i}$ em $\left[\alpha_{2}\right]$, podemos considerar $\overline{\bar{\beta}}$ como um homomorfismo de $F(k)$ em $F(k)$. Então $\beta \in\left(P_{2 k}\right)_{j n}$ induz um homomorfismo $\theta(\beta)=\overline{\bar{\beta}}: F(k) \rightarrow F(k)$ que comuta $o$ diagrama

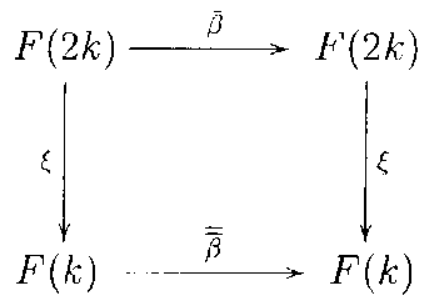

onde $\xi$ é o homomorfismo definido por $\xi\left(\alpha_{i}\right)=\alpha_{i}$ e $\xi\left(\tilde{\alpha}_{i}\right)=\alpha_{i}{ }^{1}$. Observemos o seguinte diagrama comutativo: 


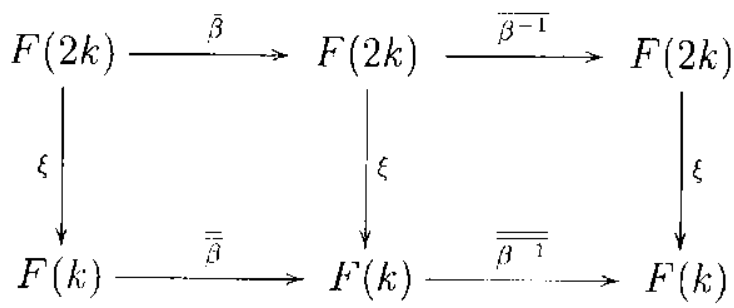

Então $\overline{\beta^{-1}} \overline{\bar{\beta}} \xi=\xi \bar{\beta}^{-\overline{1}} \bar{\beta}=\xi \bar{\beta}^{-1} \bar{\beta}=\xi$ e como $\xi$ ć sobrejetora, $\forall y \in F(k), y=\xi(x)$ para algum $x \in F^{\prime}(2 k), \log 0 \overline{\bar{\beta}^{-1}} \overline{\bar{\beta}}(y)=\overline{\overline{\beta^{1}}} \overline{\bar{\beta}} \xi(x)=\xi(x)=y$. Portanto $\overline{\overline{\beta^{-1}}} \overline{\bar{\beta}}=i d_{F(k)}$. Analogamente, $\overline{\bar{\beta}} \overline{\overline{\beta-1}}=i d_{F(k)}$. Assim $\overline{\overline{\beta^{-1}}}=\overline{\bar{\beta}}$.

Segue também do diagrama que $\overline{\bar{\beta}}$ leva cada $\alpha_{i}$ em um de seus conjugados e que $\theta:\left(l_{2 k}^{\prime}\right)_{30} \rightarrow C A_{k}$ ć um homomorfismo.

Pelo Lema 3.2.4,CA $A_{k}$ é gerado por $\alpha_{i j}$ para $i, j \in \underline{k}$ e $i \neq j$, onde $\alpha_{i j}\left(\alpha_{i}\right)=\alpha_{j} \alpha_{i} \alpha_{j}^{-1}$ e $\alpha_{i j}\left(\alpha_{l}\right)=\alpha_{l}$ se $l \neq i$

Para provarmos que $\theta$ é sobrejetora, consideremos $\beta_{i j}$, para todo $i, j \in \underline{k}$ com $i>j$, a trança pura com $2 k$ cordas representada na figura 40 . É para todo $i, j \in k$ com $i<j$, scja $\beta_{i j}$ a trança pura com $2 k$ cordas representada na figura 41 .

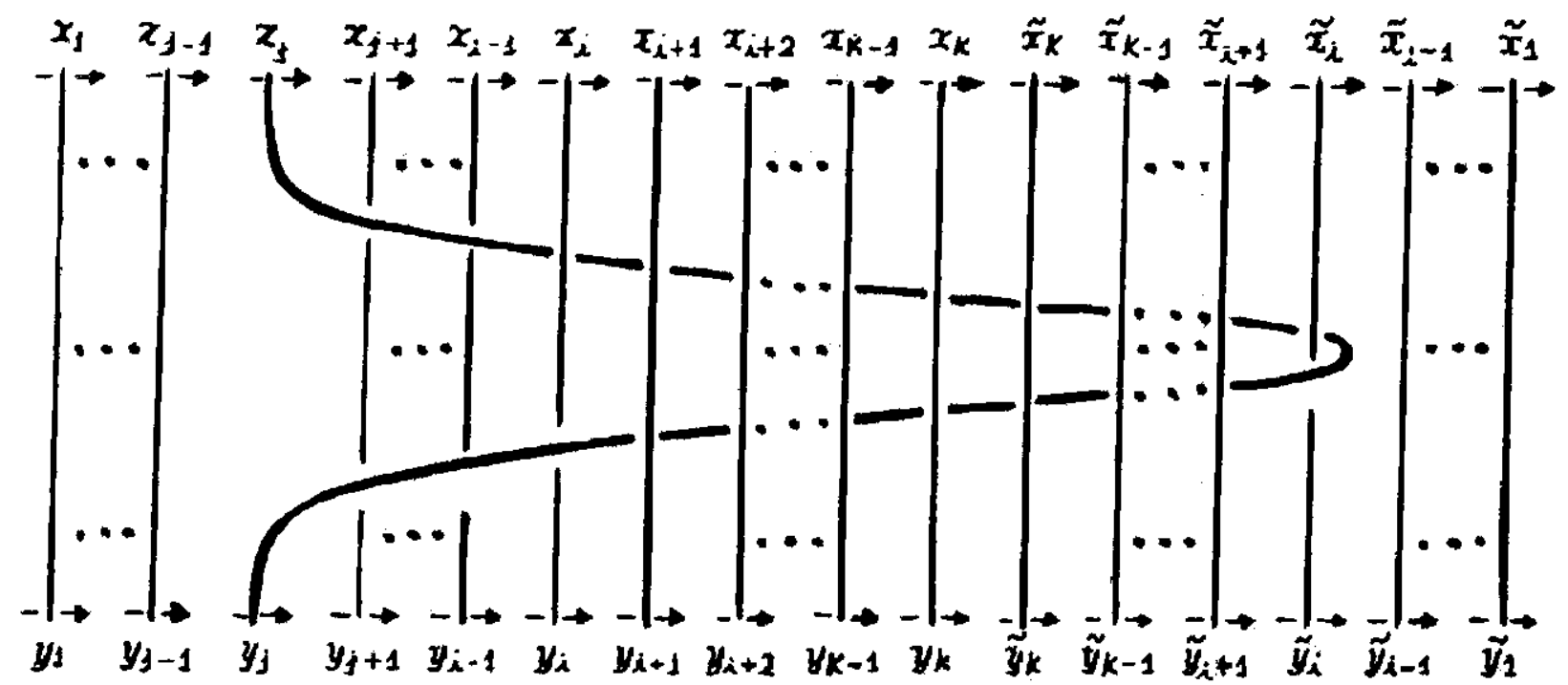

Figura 40 


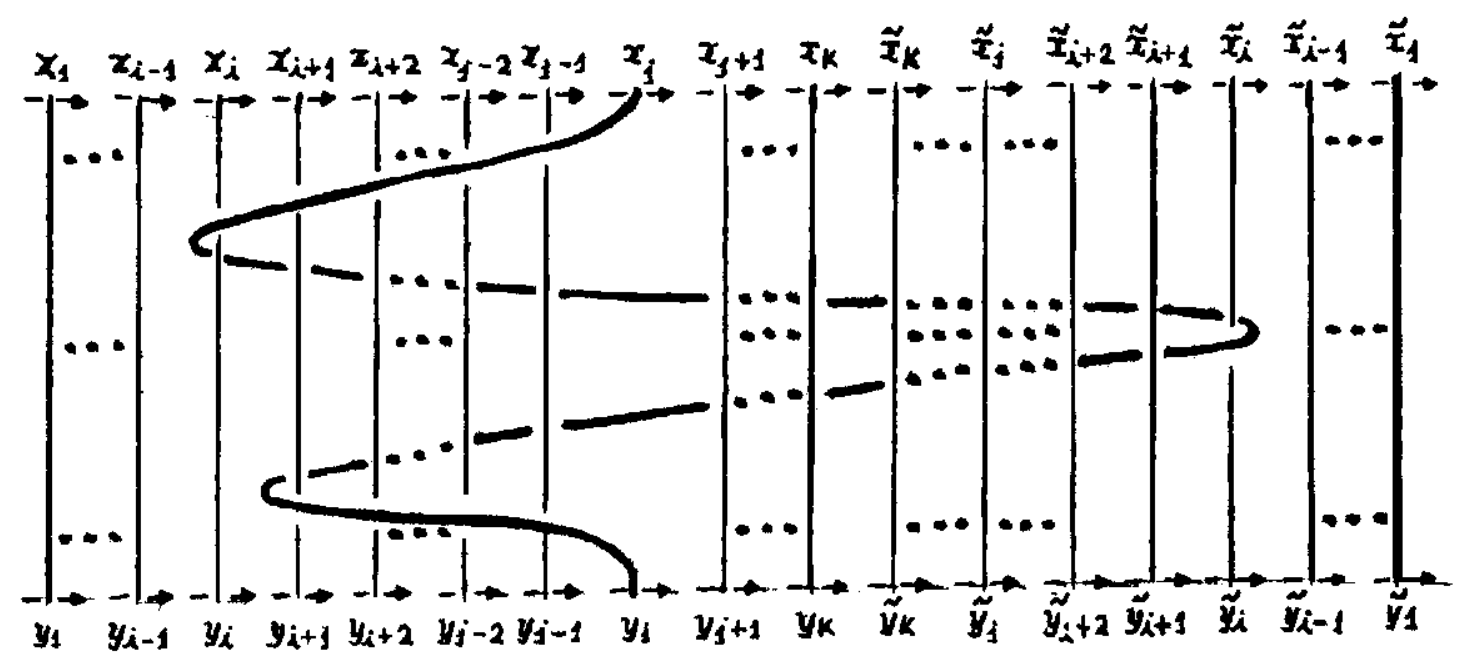

Figura 41

Vejamos que $\forall i, j \in \underline{k} \mathrm{com} i \neq j, \beta_{2 \jmath} \in\left(P_{2 k}\right)_{j 0}$. Primeiro, o caso cm que $2>j$. As setas, nas figuras abaixo, indicam o movimento a ser realizado.

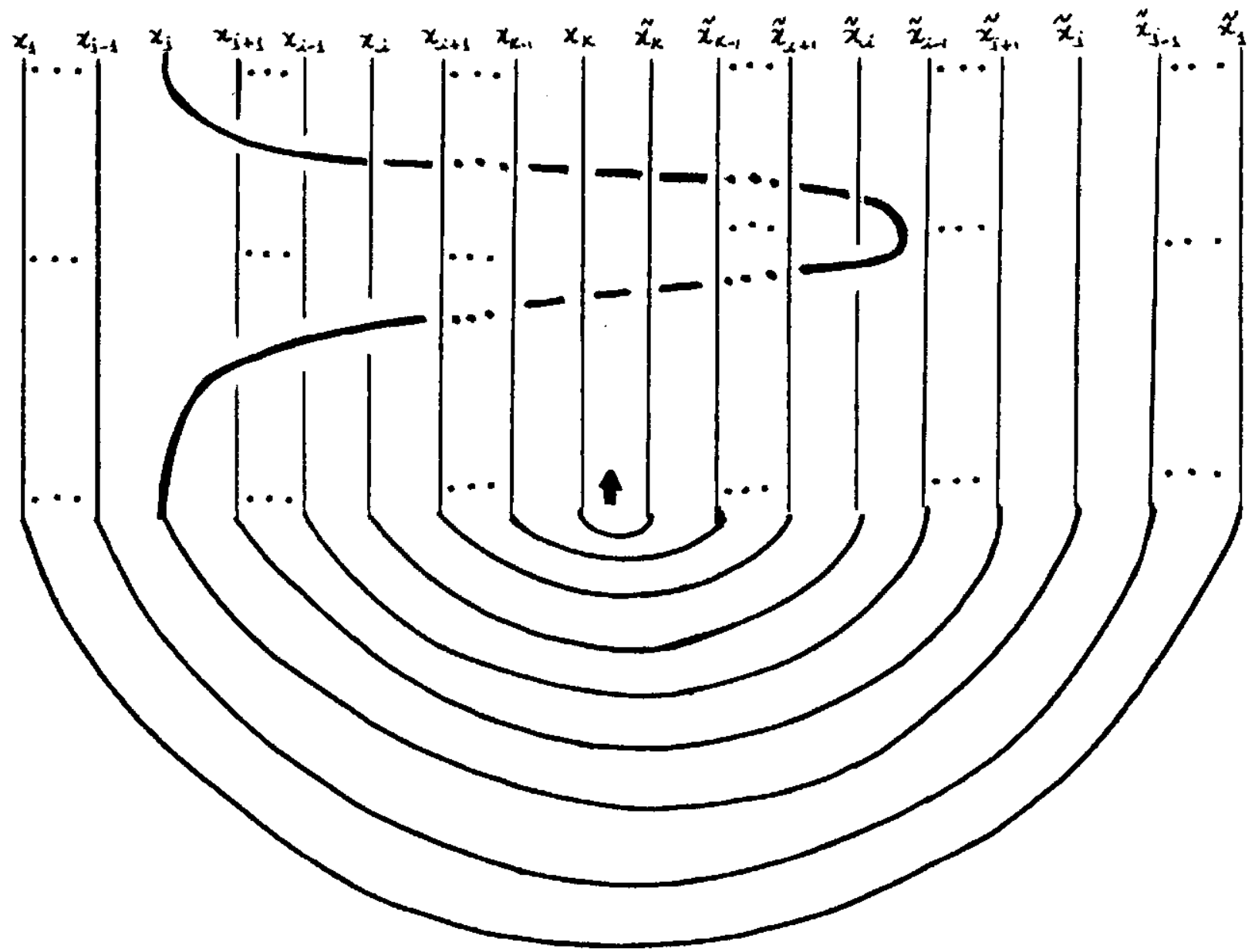



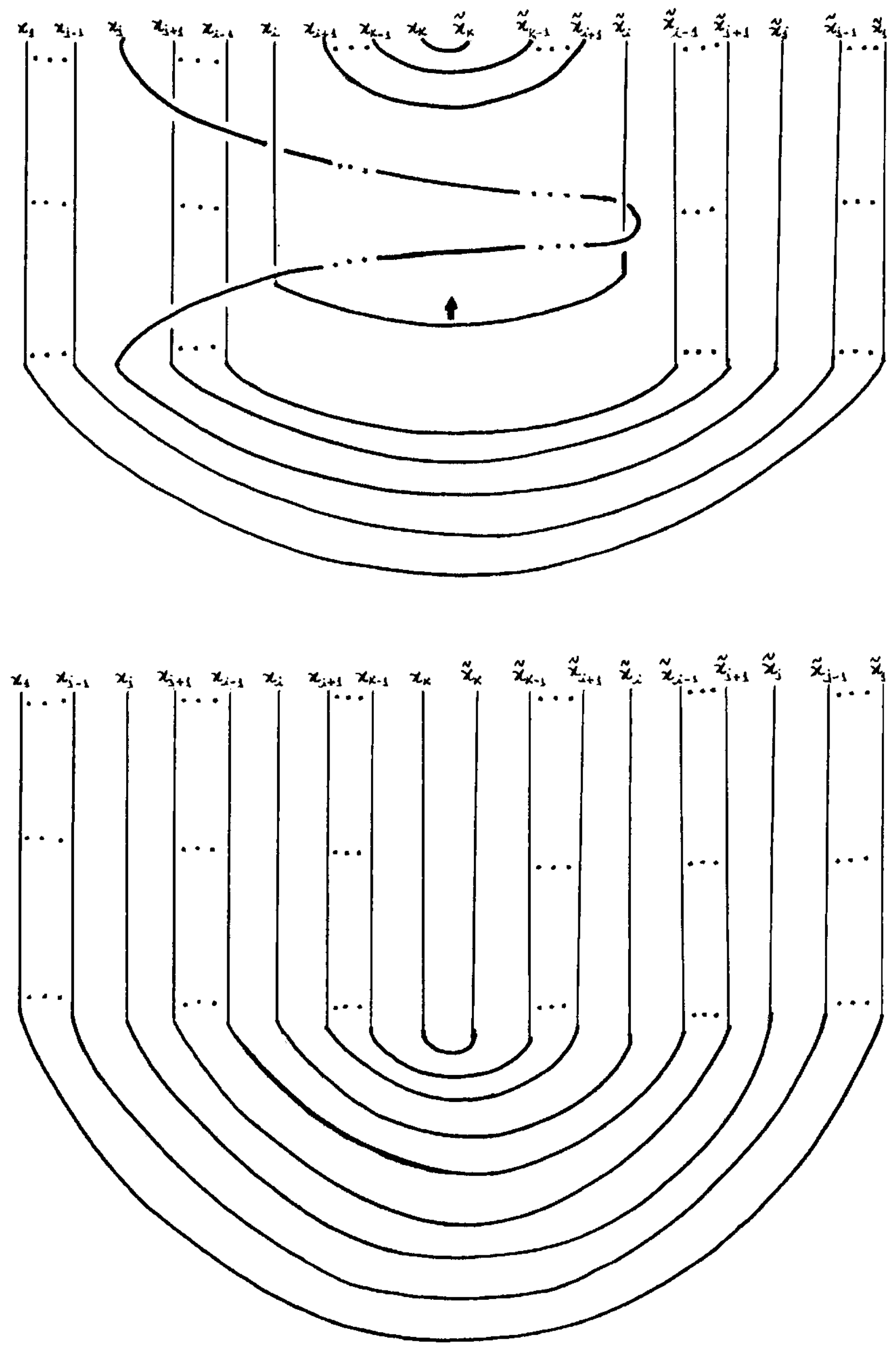

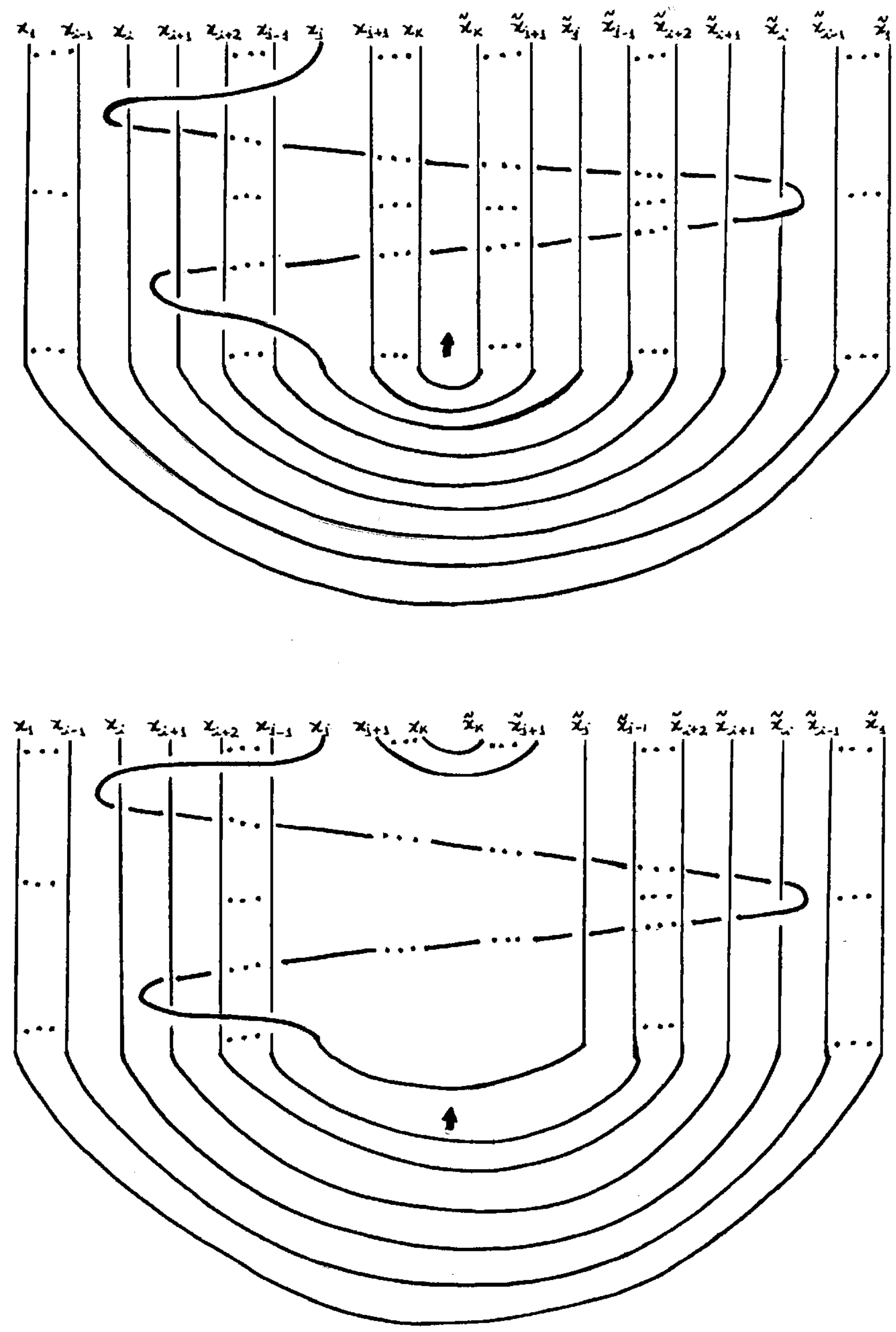

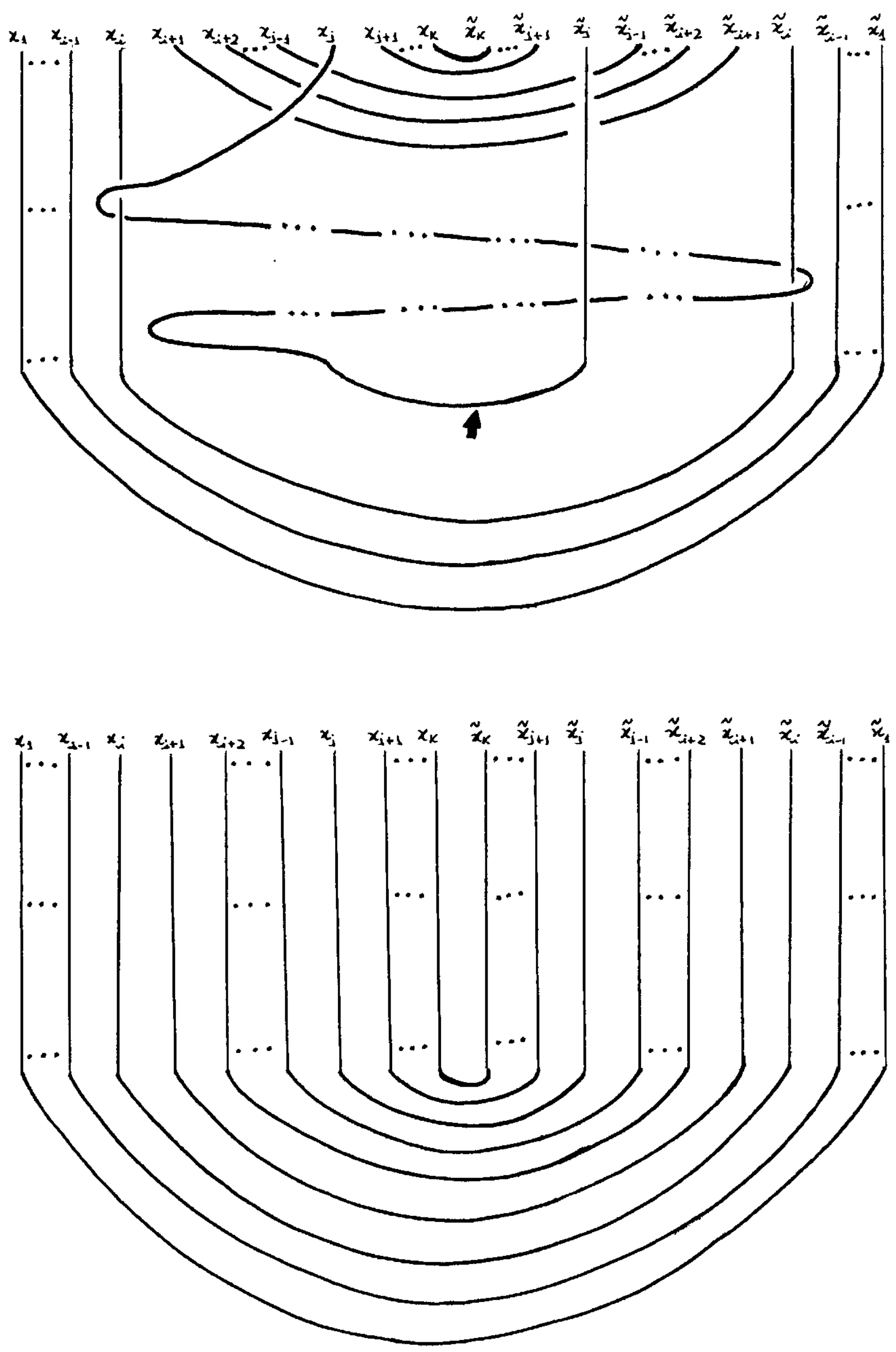
Seja $z_{i+1}=x_{i+1} x_{i+2} \ldots x_{k} \ddot{x}_{k} \ldots \bar{x}_{1+2} \tilde{x}_{i+1}$ e $z_{i}=x_{i} z_{i+1} \tilde{x}_{i}$.

Primeiro, sujonhamos que $i>j$. Neste caso, em $\Pi_{1}\left(\beta_{i j}\right)$ temos as relações:

$x_{l}=y_{l}$ se $1 \leq l<j$,

$x_{j}=z_{i} z_{i+1}^{-1} y_{j} z_{i+1} z_{i}{ }^{-1}$.

$x_{l}=x_{j}^{-1} y_{j} y_{l} y_{j}{ }^{1} x_{j}$ se $\quad j<l<i$,

$x_{i}=y_{j} y_{i} y_{j}^{-1}$,

$x_{l}=y_{l} \quad$ sc $\quad i<l \leq k$.

(Além disso, $\dot{x}_{l}=\tilde{y}_{l}$ se $1 \leq l<i$,

$\bar{x}_{\imath}=z_{2}^{-1} x_{j} z_{i} \tilde{y}_{2} z_{2}{ }^{1} x_{j}^{-1} z_{i}$

$\tilde{x}_{l}-\tilde{y}_{l}$ se $i<l \leq k$.)

Lembremos que em $\frac{\Pi_{1}\left(\beta_{i j}\right)}{\left\langle y_{i} \tilde{y}_{i} \mid i \in \underline{k}\right\rangle^{N}}$ temos $\left[\tilde{x}_{i}\right]^{1}=\left[\tilde{x}_{i}\right]$, entäo, neste grupo, obtemos as relações $\left.\left[x_{i}\right]=\mid y_{j}\right]\left[y_{i}\right]\left[y_{j}\right]^{-1}$ e, $\forall l \neq i,\left[x_{l}\right]=\left[y_{l}\right]$. Portanto, se $i>j$ temos $\theta\left(\beta_{i j}\right)=\alpha_{i j}$.

De modo análogo, se $i<j$, obtemos as seguintes relaçöes em $\mathrm{II}_{1}\left(\beta_{i j}\right)$ :

$x_{l}=y_{l} \quad$ se $\quad l<i$,

$x_{i}=x_{3} y_{i} x_{j}^{-1}$

$x_{l}=x_{j} y_{j}{ }^{1} y_{l} y_{j} x_{j}^{-1}$ se $i<l<j$,

$x_{j}=z_{i} z_{i+1}^{-1} x_{j} y_{j} x_{j}^{-1} z_{i+1} z_{i}{ }^{1}$,

$x_{l}=y_{l}$ se $j<l \leq k$.

(Além disso, $\tilde{x}_{l}=\tilde{y}_{l} \quad$ se $\quad l<i$,

$\tilde{x}_{i}=z_{i}^{-1} x_{j} z_{i} \tilde{y}_{i} z_{i}^{-1} x_{j}^{-1} z_{i}$,

$\tilde{x}_{l}=\tilde{y}_{l} \quad$ se $\quad i<l \leq k$.)

Neste caso seguc também que $\theta\left(\beta_{i j}\right)=\alpha_{i j}$.

Proposiçāo 5.3.2. $\left(P_{2 k}\right)_{j_{0}}$ atua no conjunto das classes de cobordismo bordante de $k$ enlaçamentos de intervalos bordantes.

\section{Demonstração:}

Seja $f$ um $k$-enlaçamento de intervalos bordante com meridianos $a_{1}, \ldots, a_{k}$ no topo e $b_{1}, \ldots, b_{k}$ na basc e seja $\beta \in\left(P_{2 k}\right)_{j_{0}}$. 
Usando a mesma notação da proposição anterior, o teorema de Seifert-Van Kampen nos fornece o seguinte diagrama push-out

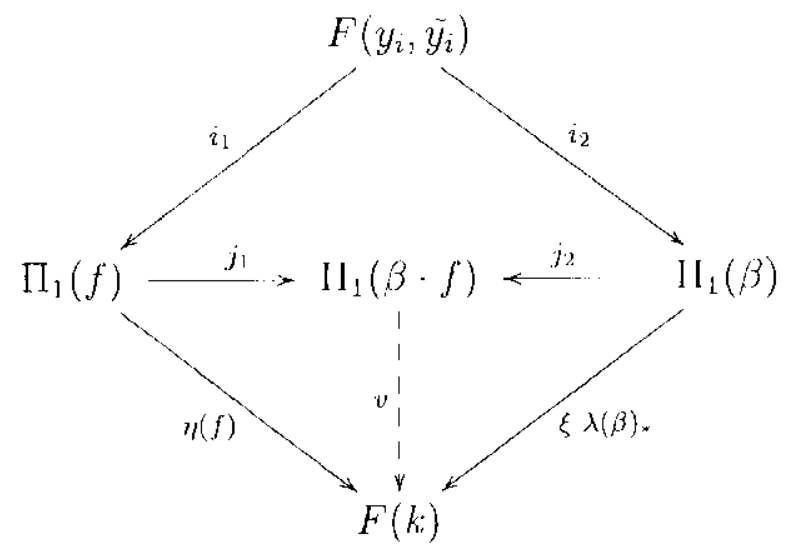

onde $F\left(y_{i}, \tilde{y}_{2}\right)$ é o grupo livre gerado por $y_{1}, \ldots, y_{k}, \tilde{y}_{k}, \ldots, \tilde{y}_{1}, i_{2}$ é o homomorfismo inclusão, $j_{1}$ c $j_{2}$ são induzidas pelas aplicações inclusão, $i_{1}$ c o homomorfismo definido por $i_{1}\left(y_{i}\right)=a_{i}$ e $i_{1}\left(\tilde{y}_{i}\right)=b_{i}^{-1}$, para todo $i \in \underline{k}$, e $v: \Pi_{1}(\beta \cdot f) \rightarrow F(k)$ é o homomorfismo que comuta o diagrama push-out.

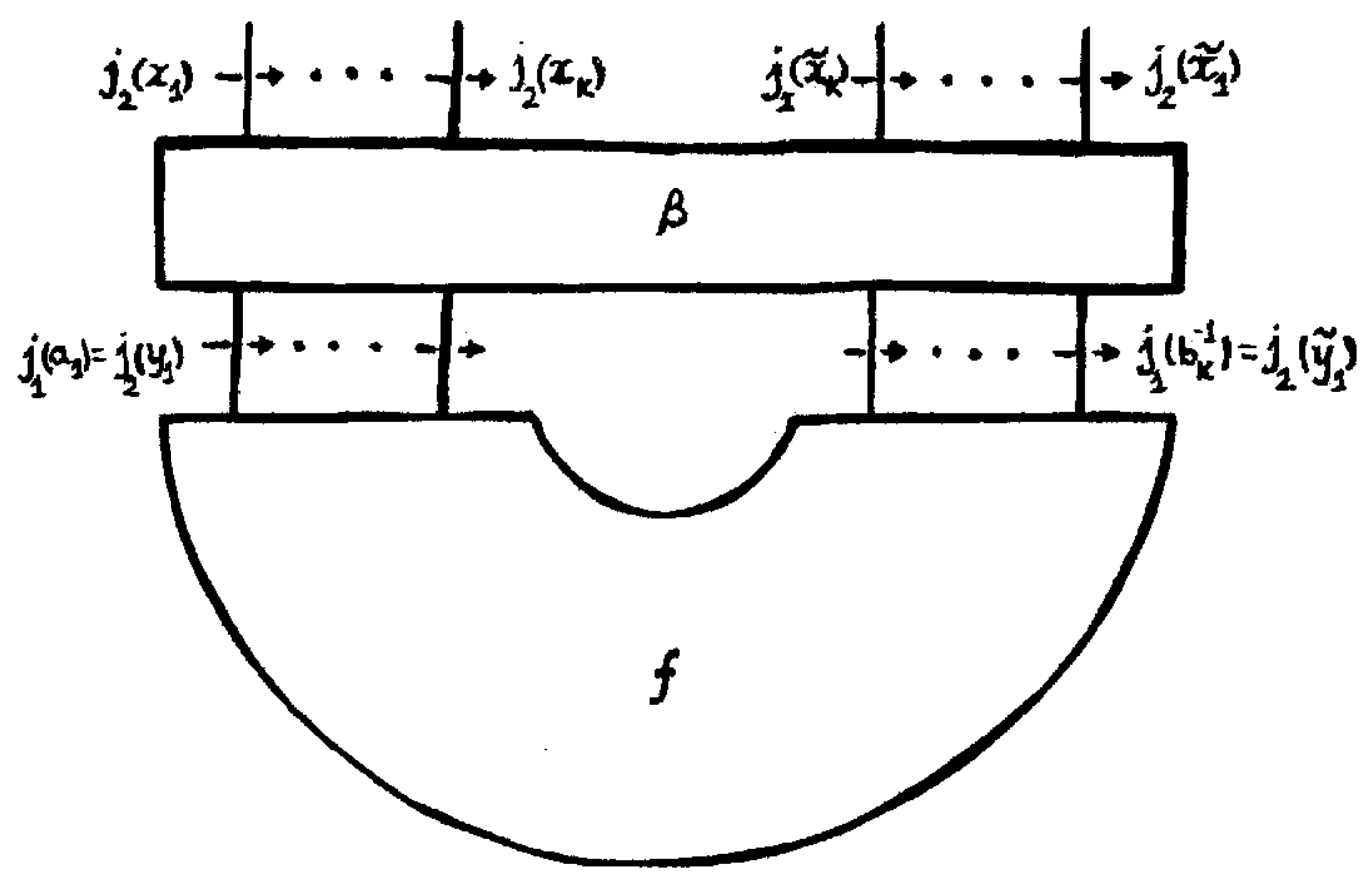

Figura 43

Para todo $i \in k, v j_{2}\left(x_{i}\right)=\xi \lambda(\beta)_{*}\left(x_{i}\right)=\xi \bar{\beta}\left(\alpha_{i}\right)=\overline{\bar{\beta}}\left(\alpha_{i}\right)$ e $v j_{2}\left(\bar{x}_{i}\right)=\xi \lambda(\beta)_{*}\left(\bar{x}_{i}\right)=$ $\xi \bar{\beta}\left(\tilde{\alpha}_{i}\right)=\overline{\bar{\beta}}\left(\alpha_{i}\right)^{-1}$. Então $\overline{\bar{\beta}}^{-1} v$ é urn splitting para $\beta \cdot f$, assim $\beta \cdot f$ é um enlaçamento de 
intervalos bordante. Alóm disso, suponhamos que $g$ ć outro $k$-enlaçamento de intervalo bordante e $C$ é um cobordismo entre $f$ e $g$. Substituindo $f$ por $C$ e $\beta$ por $\beta$ x $i d_{I}$ no diagrama push-out anterior, obtemos um splitting para o cobordismo $\left(\beta \mathrm{x} i d_{I}\right) \cdot C$ entre $\beta \cdot f e \beta \cdot g$.

Seja $<\beta_{i j} \mid i, j \in k, i \neq j>0$ subgrupo de $\left(P_{2 k}\right)_{, 0}$ gerado por $\beta_{i j}$ definido na Proposição 5.2.1.

Teorema 5.3.3. Qualquer splitting de um enlaçamento bordante $I$ com $k$ componentes pode ser obtido agindo-se em qualquer $k$-enlaçamento de intervalos bordante $f$, com $\hat{f}=L$, por um elemento de $\left\langle\beta_{i j}\right| i, j \in \underline{k}, i \neq j>$ e fechando-se o enlaçamento de interwalos bordante resultante.

\section{Demonstração:}

Seja $f$ um k-tnlaçamento de intervalos bordante com $\hat{f}=L . \eta=\widehat{\eta(f)}$ é um splitting para $L$. Se um epimorfismo $z: \Pi_{1}(L) \rightarrow F(k)$ é um splitting para $L$ então $z=\alpha \eta$, para algurm $\alpha \in C A_{k}$. Agora, $\widehat{\beta_{i j} \cdot \hat{f}}=\hat{f}=L$ e $\eta\left(\widehat{\beta_{i j} \cdot f}\right)$ restrita aos geradores de $\Pi_{1}\left(\widehat{\beta_{i j} \cdot f}\right)$, cujos representantes estäo no complemento de $f$ em $B_{-.}$, coincide com $\overline{\bar{\beta}}_{i j}{ }^{1} \widehat{\eta(f)}$, pela Proposição 5.2 .2 e os $\overline{\bar{\beta}}_{\imath j}=\alpha_{i j}$ geram $C^{r} A_{k}$.

Exemplo 5.3.4. Scja $L$ o seguinte cnlaçamento bordante orientado:

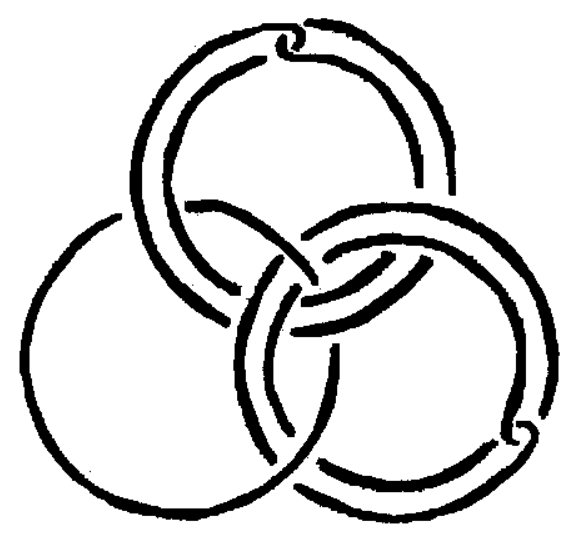

Figura 44a 
Considercmos uma d-base para $L$.

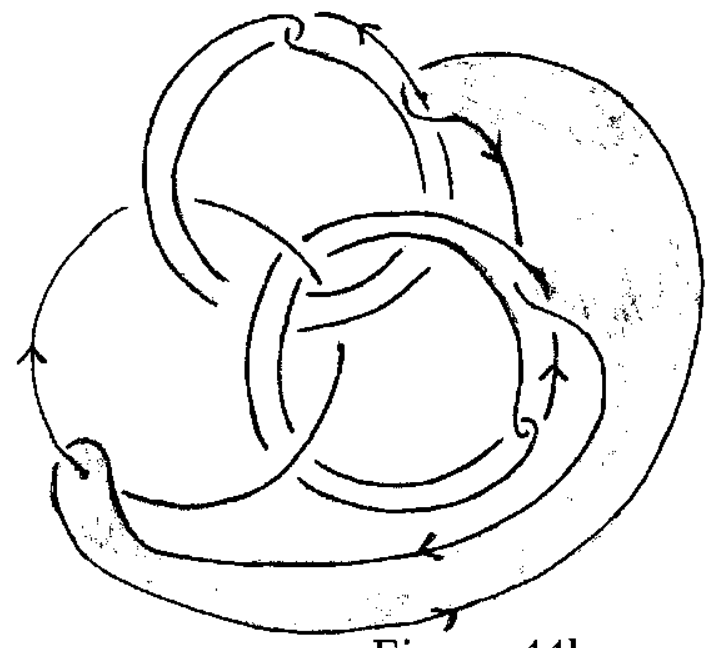

Figura $44 \mathrm{~b}$

Logo, pela bijeção de llabegger-Lin, ao enlaçamento d-baseado acima corresponde um enlaçamento de intervalos, dado na figura. 44e.

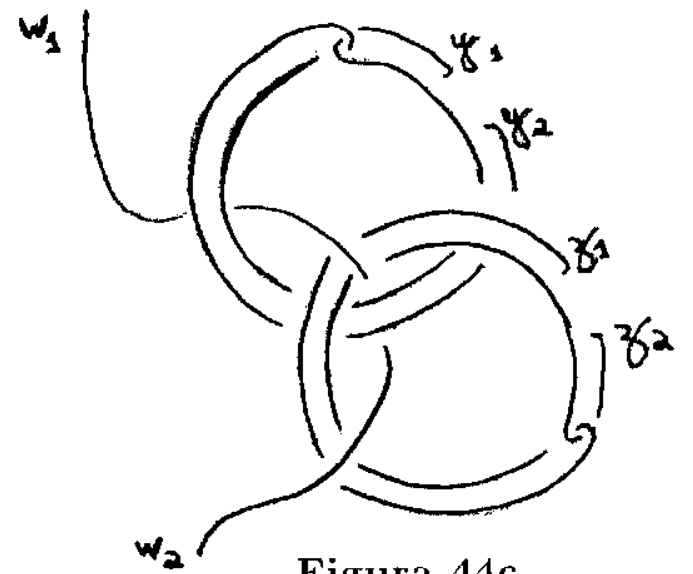

Figura 44c

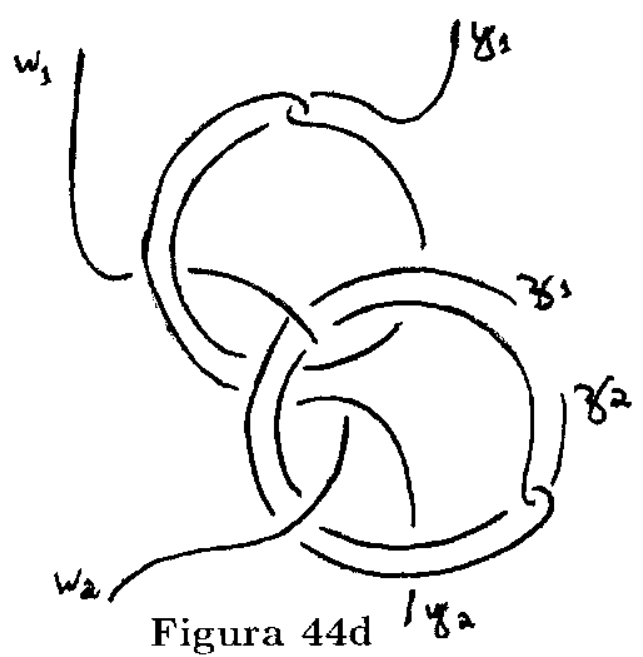

Figura 44d 182

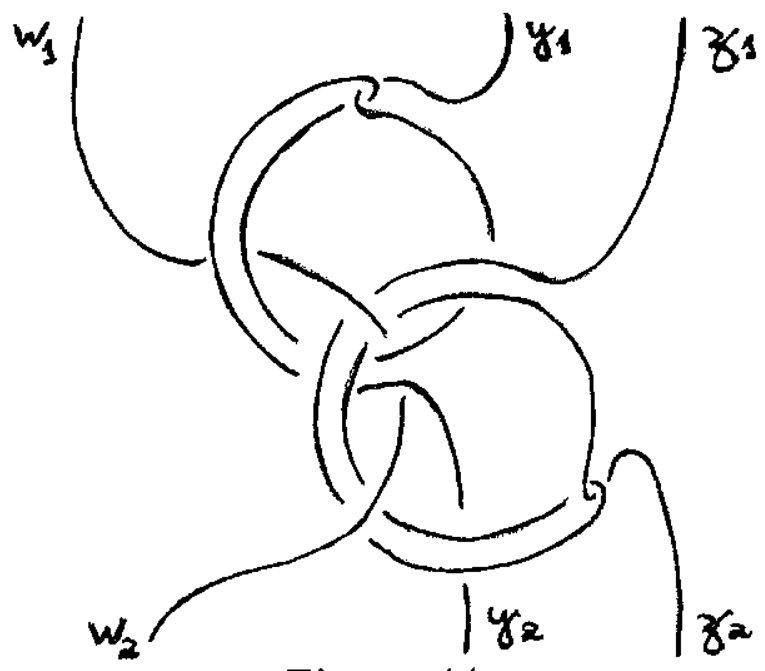

Figura 44e 
Como o enlaçamento acima tem 3 componentes, consideremos o grupo $<\beta_{i j}, i, j \in$ $\underline{3}, i \neq j>$. Estes elementos $\beta_{i j}$ estão representados nas figuras abaixo.

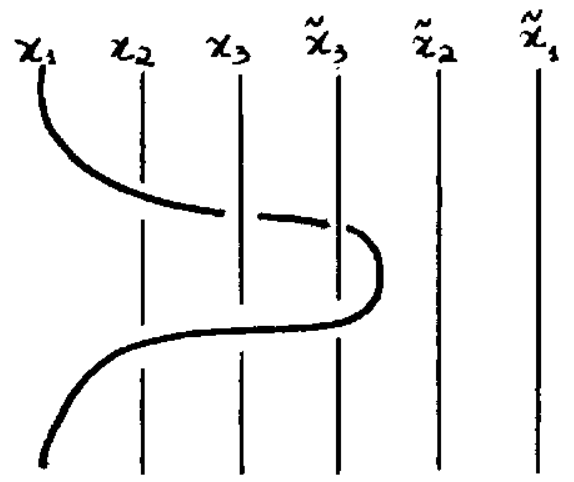

Figura 44f: $\beta_{3,1}$

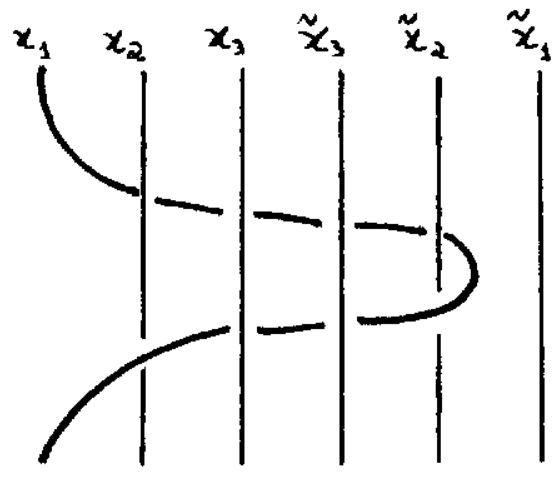

Figura 44h: $\beta_{2,1}$

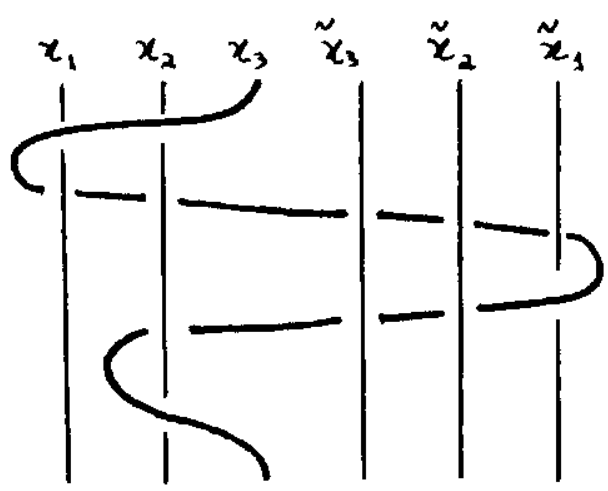

Figura $44 \mathrm{j}: \beta_{1,3}$

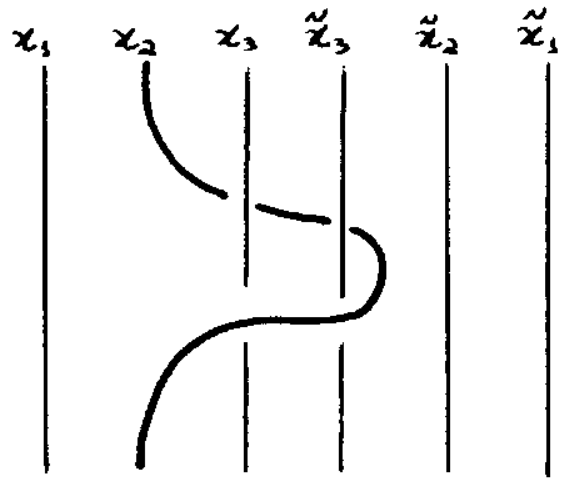

Figura $44 \mathrm{~g}: \beta_{3,2}$

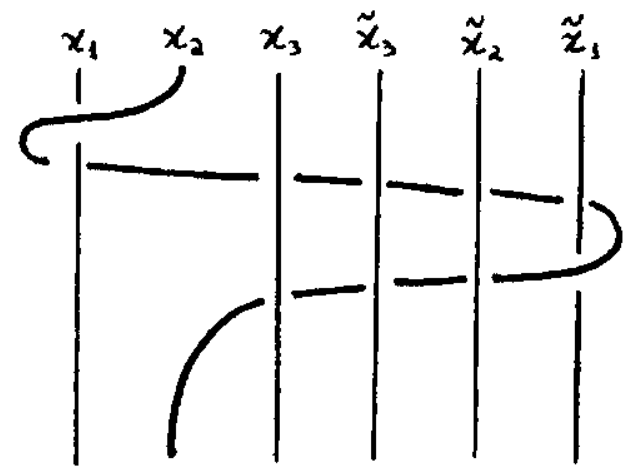

Figura 44i: $\not 1,2$

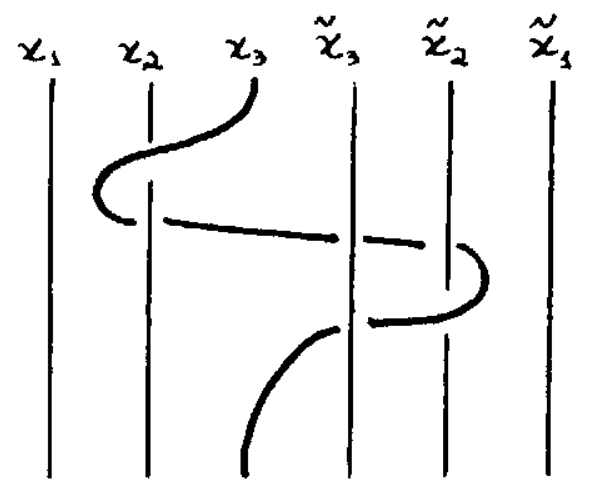

Figura 441: $\beta_{2,3}$ 
Atuando-se com as tranças $\beta_{i j}, i \neq j, \forall i, j \in \underline{3}$, no enlaçamento de intervalos bordante 44 e e fechando-se o enlaçamento resultante obtcmos o enlaçamento inicial $L$, uma vez que os $\beta_{i j}$ (e portanto os $\beta_{i j}{ }^{1}$ ) pertencem a $\left(P_{2 k}\right)_{j_{0}}$. Vejamos a açāo de $\beta_{3,2}$ no enlaçamento de intervalos $44 e$.

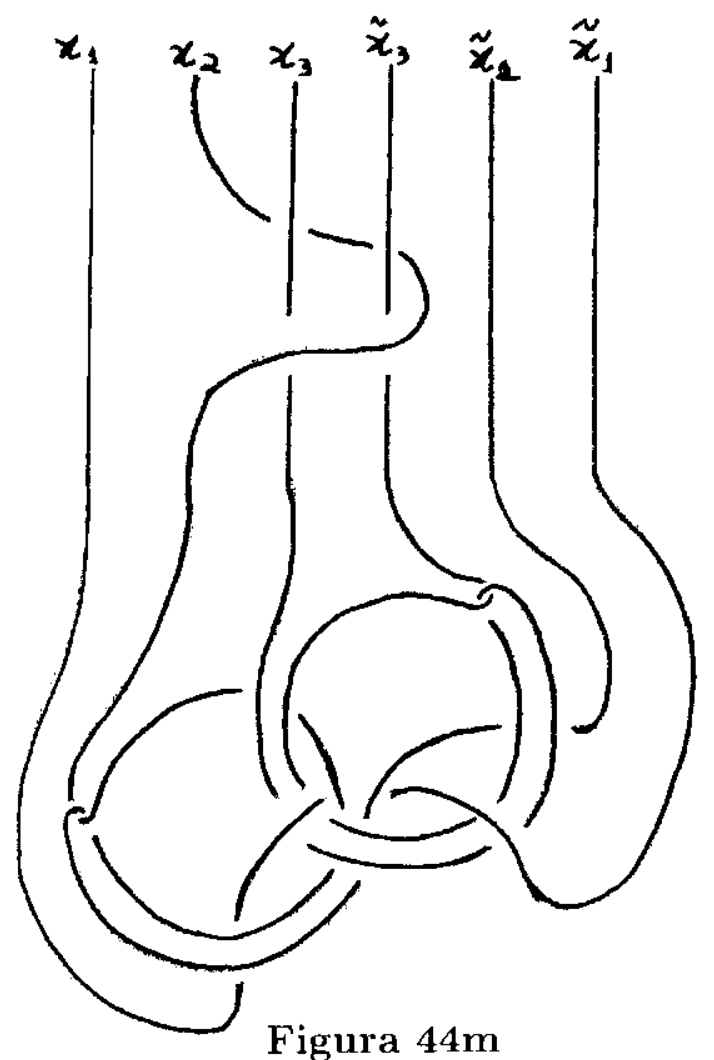

44c possui una aplicação splitıing que leva $w_{1}$ em $x, y_{1}$ em $y$ e $z_{1}$ cm $z$ onde $x, y, z$ são os geradores do grupo livre $F(3)$. Notemos que no enlaçamento de intervalos bordante obtido em $44 m$, o splitting leva $x_{1}$ em $x, x_{2}$ em $y$ e $x_{3}$ em $z$, além disso, valem as seguintes rclações:

$$
w_{1}=x_{1}, \quad y_{1}=\tilde{x}_{3}^{-1} x_{3}^{-1} x_{2} x_{3} \tilde{x_{3}} \quad \text { e } \quad z_{1}-y_{1}{ }^{1} x_{3} y_{1}
$$

Assim, por esta aplicação splitting, $w_{1}$ é levado em $x, y_{1}$ em $z z^{1} y z z^{-1}=y$ e $z_{1}$ é levado em $y^{-1} z y$. 


\section{Referências Bibliográficas}

[1] BIRMAN, J.S., Braids, Links and Mapping Class Groups, Annals of Mathematics Studies 82, Princeton University Press, (1975).

[2] BURDE, G., ZIESCHANG, H., Knots, Walter de Gruter, Berlin - New York, (1985).

[3] CAPPELI., S.E., SHANESON, J.L., Link Cobordism, Comment. Math. Helvetici 55 (1980), 20-49.

[4] COHEN, D.E., Combinatorial Group theory: a lopological approach, London Mathematical Society. Student, 'Texts 14(1989).

15] DE CAMPOS, J.E.P.P., Boundary String Links, Journal of Knot Theory and its Ramifications, vol 08, n: 07, 855-878, (1999).

[6] DE CAMPOS, J.E.P.P., Boundary String Links, Ph.D. Dissertation, Brandeis University (1998).

[7] GLTIÉRREZ, M. A., Boundary links and unlinking theorem, Transactions of the American Mathematical Society, vol 03, 451-499, (September 1987).

[8] HABBEGER, N.; LIN X.S., The classification of links, Journal of the American Mathematical Society, vol 03, n: 02 (April 1990).

[9] HANSEN, V.L., Braids and Coverings: Cambridge Eniversity Press, (1989).

[10] KO, K.H., Seifert matrices and boundary link cobordism, Transactions of the American Mathematical Society, vol 299, n: 02 (February 1987).

[11] LE DIMET,J.Y., Cobordism d'enlacements de disques, Memoire de la Société Mathématique de France, 32, Supplément au Bulletin de la S.M.F., Tome 116, fasricule 2 (1988). 
[12] MASSEY, W.S., Algebraic Topology: An Introduction, Harcourt, Brace \& World, Inc. (1967).

[13] ROFSEN, D., Knots and Links, Publish or Perish, Inc. (1990).

[14] SMYTHE, N., Wisconsin Topology Seminar, Ann. of Math. Studies, n: 60, Princeton University Press, Princeton, N.Y., (1995). 